\title{
Isospin Physics in Heavy-ion Collisions at Intermediate Energies
}

\author{
Bao-An Lif ${ }^{a}$, Che Ming Kofa and Wolfgang Bauer ${ }^{\mathrm{b}}$ \\ ${ }^{a}$ Department of Physics and Cyclotron Institute \\ Texas A\&M University, College Station, TX 77843-3366, USA \\ ${ }^{b}$ National Superconducting Cyclotron Laboratory \\ and Department of Physics and Astronomy \\ Michigan State University, East Lansing, MI 48824-1321, USA
}

\begin{abstract}
In nuclear collisions induced by stable or radioactive neutron-rich nuclei a transient state of nuclear matter with an appreciable isospin asymmetry as well as thermal and compressional excitation can be created. This offers the possibility to study the properties of nuclear matter in the region between symmetric nuclear matter and pure neutron matter. In this review, we discuss recent theoretical studies of the equation of state of isospin-asymmetric nuclear matter and its relations to the properties of neutron stars and radioactive nuclei. Chemical and mechanical instabilities as well as the liquid-gas phase transition in asymmetric nuclear matter are investigated. The in-medium nucleon-nucleon cross sections at different isospin states are reviewed as they affect significantly the dynamics of heavy ion collisions induced by radioactive beams. We then discuss an isospin-dependent transport model, which includes different mean-field potentials and cross sections for the proton and neutron, and its application to these reactions. Furthermore, we review the comparisons between theoretical predictions and available experimental data. In particular, we discuss the study of nuclear stopping in terms of isospin equilibration, the dependence of nuclear collective flow and balance energy on the isospin-dependent nuclear equation of state and cross sections, the isospin dependence of total nuclear reaction cross sections, and the role of isospin in preequilibrium nucleon emissions and subthreshold pion production.
\end{abstract}

\footnotetext{
${ }^{1}$ email: Bali@comp.tamu.edu

${ }^{2}$ email: Ko@comp.tamu.edu

3 email: Bauer@nscl.msu.edu
} 


\section{Contents}

\begin{tabular}{lll}
\hline & Introduction & 2 \\
\hline
\end{tabular}

2 Isospin dependence of the nuclear equation of state 4

2.1 Isospin dependence of the nuclear matter compressibility, binding en-

ergy, and density at saturation . . . . . . . . . . . . 5

2.2 Empirical parabolic law and the nuclear symmetry energy . . . . . . 11

$2.3 \quad$ Neutron stars and the EOS of asymmetric nuclear matter . . . . . . . 16

2.4 Radioactive nuclei and the EOS of asymmetric nuclear matter . . . . . 21

2.5 Chemical and mechanical instabilities in hot asymmetric nuclear matter 26

$2.6 \quad$ New features of liquid-gas phase transition in asymmetric nuclear matter 37

3 Isospin dependence of in-medium nucleon-nucleon cross sections 41

3.1 Isospin dependence of free-space NN cross sections . . . . . . . . . . . 41

3.2 Isospin dependence of in-medium NN cross sections . . . . . . . . . . 43

4 Isospin-dependent phenomenology in heavy-ion collisions at intermediate energies $\quad 46$

4.1 Isospin-dependent transport models for heavy-ion collisions . . . . . . 46

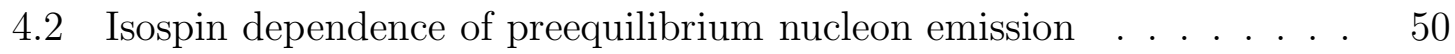

4.3 Isospin equilibrium as a probe of nuclear stopping power . . . . . . . 61

4.4 Isospin dependence of nuclear collective flow . . . . . . . . . . 69

4.5 Isospin dependence of total reaction cross sections and radii of neutronrich nuclei . . . . . . . . . . . . . . . . . . . . . . . . . 79

$4.6 \quad$ Isospin dependence of subthreshold pion production . . . . . . . . . . 84

$\begin{array}{llr}5 \text { Overview and perspectives } & 90\end{array}$

\begin{tabular}{lll}
\hline 6 & Acknowledgement & 92
\end{tabular} 


\section{Chapter 1}

\section{Introduction}

Recent advances in experiments using radioactive beams with large neutron or proton excess have made it possible to create not only nuclei at the limits of stability, but also a transient state of nuclear matter with appreciable isospin asymmetry, thermal excitation, and compression. Extensive reviews have been written on the properties of these nuclei, and they can be found in Refs. [1, 2, 3, 4, 5, 6, 7, 8]. In this article we will review instead the role of the isospin degree of freedom in heavy-ion collisions at intermediate energies. The main goal of such studies is to probe the properties of nuclear matter in the region between symmetric nuclear matter and pure neutron matter. This information is important in understanding the explosion mechanism of supernova and the cooling rate of neutron stars. For example, the prompt shock invoked to understand the explosion mechanism of a type II supernova requires a relatively soft equation of state ( EOS) [9], which can be understood in terms of the dependence of the nuclear compressibility on isospin. In the model for prompt explosion [10], the electron-capture reaction drives the star in the latest stage of collapse to an equilibrium state where the proton concentration is about $1 / 3$, which, according to microscopic many-body calculations, reduces the compressibility by about $30 \%$ compared to that for symmetric nuclear matter. Moreover, the magnitude of proton concentration at $\beta$ equilibrium in a neutron star is almost entirely determined by the isospin-dependent part of the nuclear EOS, i.e., the symmetry energy. The proton fraction affects not only the stiffness of the EOS but also the cooling mechanisms of neutron stars [11, 12] and the possibility of kaon condensation $\left(e^{-} \rightarrow K^{-} \nu_{e}\right)$ in dense stellar matter 13. If the proton concentration is larger than a critical value of about $15 \%$, the direct URCA process $\left(n \rightarrow p+e^{-}+\bar{\nu}_{e}, p+e^{-} \rightarrow n+\nu_{e}\right)$ becomes possible, and would then enhance the emission of neutrinos, making it a more important process in the cooling of a neutron star [1].

Usually, the properties of asymmetric nuclear matter are inferred from that of symmetric and pure neutron matter by using a parabolic approximation [14], and this seems to be supported by studies based on detailed many-body calculations. Although different assumptions have been introduced in these theoretical approaches, they all predict similar results for the asymmetric nuclear matter. In particular, the density and compressibility at saturation are predicted to decrease as the nuclear matter becomes more neutron-rich. Also, the instability that leads to the liquid-gas 
phase separation in asymmetric nuclear matter is found to arise from the chemical instability due to fluctuations in isospin-asymmetry instead from the mechanical instability as a result of fluctuations in baryon density as in symmetric nuclear matter [15]. Furthermore, the nature of liquid-gas phase transition in asymmetric nuclear matter is predicted to be different from that in symmetric nuclear matter. For example, Müller and Serot have recently shown that the liquid-gas phase transition is second order rather than first order as in symmetric nuclear matter [16].

To relate the theoretical predictions to the experimental observations in heavy ion collisions with radioactive beams, the isospin-dependent nuclear transport model, which uses different mean-field potentials and cross sections for the proton and neutron, has been found to be very successful. Based on this model, it has been shown that many observed phenomena are mainly determined by the isospin-dependent nuclear equation of state and in-medium nucleon-nucleon cross sections. Other models such as the percolation and lattice gas models have also been found useful in understanding some of the phenomena. We shall review the applications of these models in studying several topics of isospin physics. These include the equilibration of the isospin degree of freedom as a probe of nuclear stopping, the isospin dependence of the nuclear collective flow and balance energy, preequilibrium nucleon emissions, and subthreshold pion production as well as the determination of the radii of neutron-rich nuclei from the total nuclear reaction cross sections, An overview and outlook will be given at the end. 


\section{Chapter 2}

\section{Isospin dependence of the nuclear equation of state}

Since the early work of Brueckner et al. [14] and Siemens [17] on isospin asymmetric nuclear matter in the late 60's, there have been many studies on this subject based on different two-body and three-body forces or interaction Lagrangians. These include the non-relativistic Brueckner approach [18, 19, 20], relativistic Brueckner approach [21, 22, 23, 24], variational many-body approach [25, 26, 27], relativistic mean-field theory [15, 23, 28, 29, 30, 31, 32], relativistic and non-relativistic Hartree-Fock [33, 34, 35] or Thomas-Fermi approximation [36, 37, and the chiral sigma model 38. Instead of discussing details of these models and their predictions, we review the most important, common features of the EOS of asymmetric nuclear matter predicted by these models. Also, we shall point out the most obvious, qualitative difference among the model predictions. For example, at densities greater than the normal nuclear saturation density, it may become important to include the relativistic effects [39, which seem already to be needed in obtaining the correct nuclear matter binding energy and saturation density. In addition, causality is violated at high densities in many non-relativistic models. We shall therefore also distinguish the predictions between relativistic and non-relativistic approaches.

Fig. 2.1 and Fig. 2.2 show three typical predictions for the EOS of asymmetric nuclear matter from the non-relativistic Skyrme-Hartree-Fock (SHF) model using the parameter set SIII [40], the relativistic mean-field (RMF) model using the parameter set TM1 [32] and the relativistic Brueckner-Hartree-Fock approach with or without the momentum-dependent self-energies [24]. The isospin asymmetry is indicated for each curve by the neutron to proton ratio $\rho_{n} / \rho_{p}$ in Fig. 2.1 and the relative neutron excess

$$
\delta \equiv \frac{\rho_{n}-\rho_{p}}{\rho_{n}+\rho_{p}} \equiv \frac{\rho_{n}-\rho_{p}}{\rho}
$$

in Fig. 2.2. A common prediction from these studies is that the asymmetric nuclear matter is less stiff and bound at saturation. The minimum in the equation of state, i.e., the energy per nucleon versus density, disappears before the pure neutron matter limit is reached, and the compressibility at saturation thus decreases as nuclear matter 


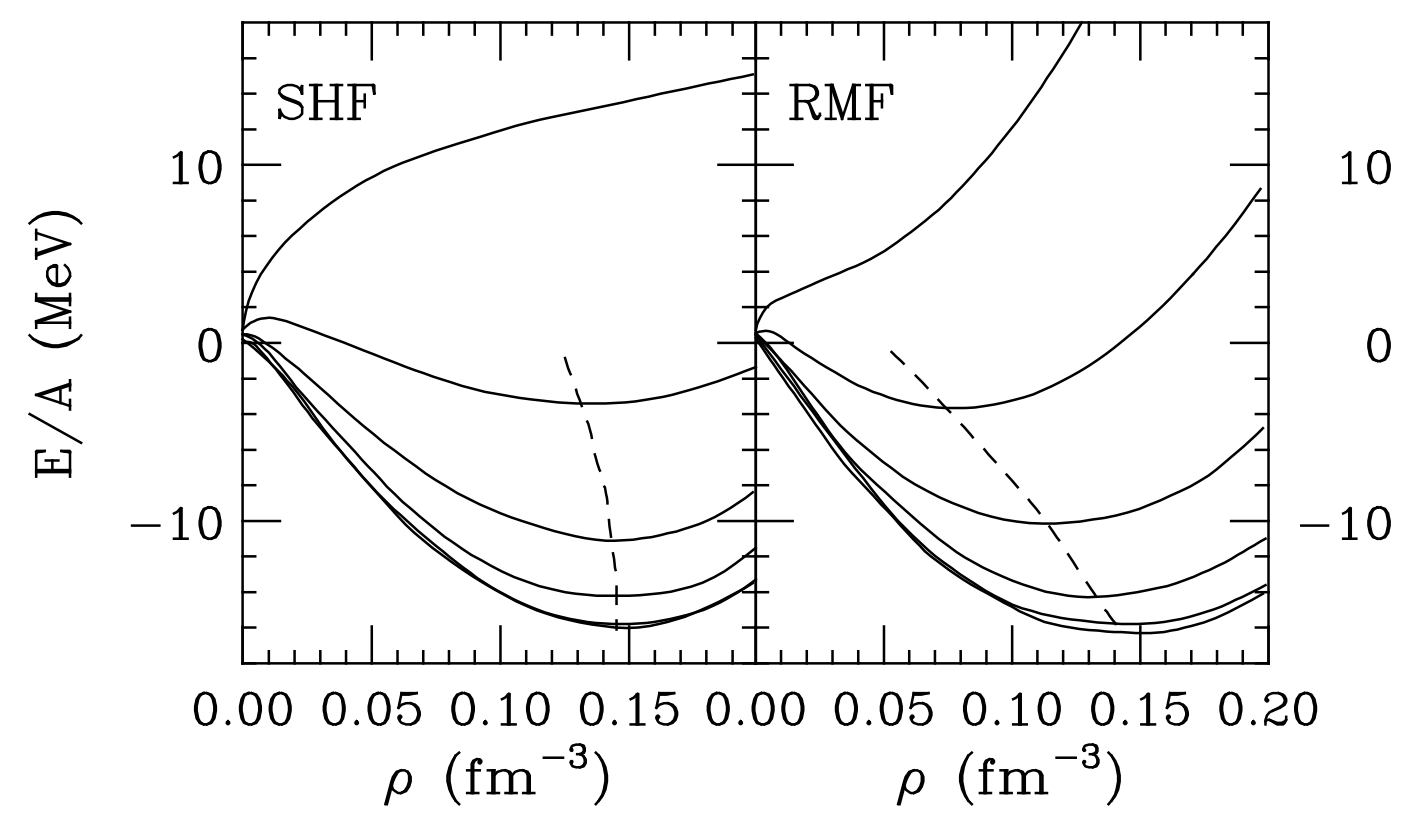

Figure 2.1: The equation of state of asymmetric nuclear matter from the SkyrmeHartree-Fock (left) and relativistic mean-field (right) model calculations. The solid curves correspond to neutron-to-proton ratios, $\rho_{n} / \rho_{p}$, of (from top to bottom) $0,0.2$, 0.4, 0.6, 0.8, and 1. Results taken from Ref. [40].

becomes more neutron-rich. Also, the saturation density is generally reduced with increasing neutron/proton ratio. Although all these models give the correct saturation properties for symmetric nuclear matter, their predictions for the EOS of asymmetric nuclear matter, such as the saturation density, are quantitatively different, In the SHF model the saturation density depends weakly on the isospin asymmetry, while in the RMF model the dependence is much stronger. This difference, as we shall see in section 2.4, results in significant differences in the density distribution and the thickness of neutron skins of radioactive nuclei. More detailed comparisons among results from the relativistic mean-field theory, relativistic Brueckner Hartree-Fock approach, and Skyrme-Hartree-Fock approach can be found in Refs. [23, 41].

\subsection{Isospin dependence of the nuclear matter com- pressibility, binding energy, and density at sat- uration}

From the EOS of asymmetric nuclear matter, i.e, the energy per nucleon $e(\rho, \delta) \equiv E / A$, the compressibility can be calculated from

$$
K(\rho, \delta)=9 \frac{\partial P(\rho, \delta)}{\partial \rho},
$$




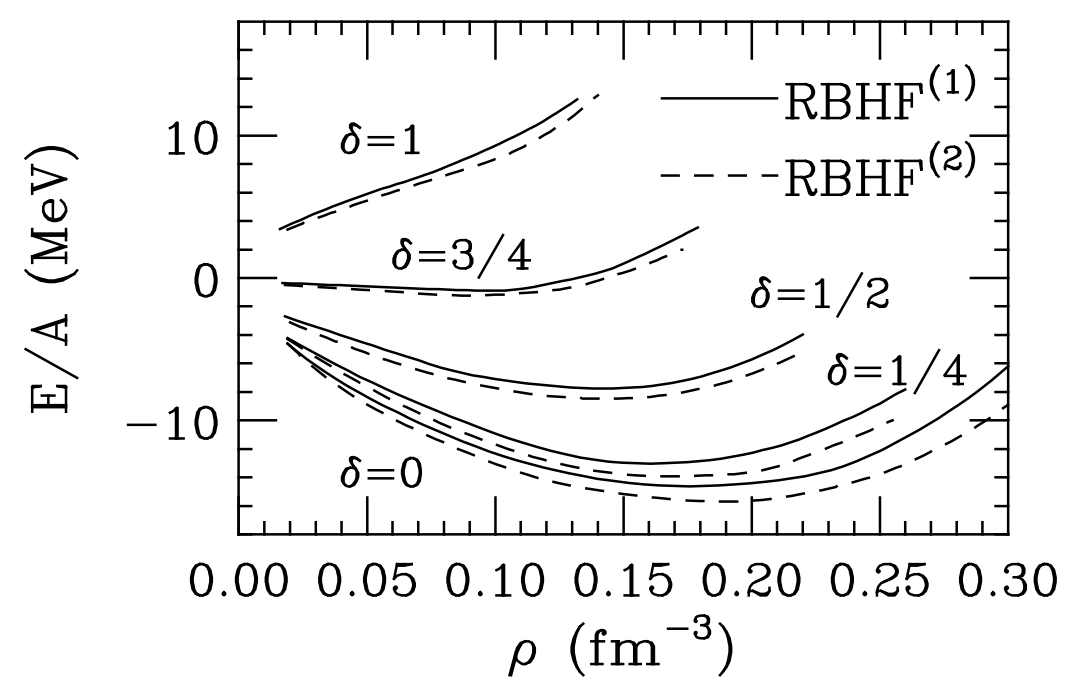

Figure 2.2: Same as Fig. 2.1 from the relativistic Brueckner-Hartree-Fock calculations. The solid and dashed curves correspond, respectively, to the case with and without momentum dependent self-energies. Taken from Ref. [24].

where the pressure $P$ is given by

$$
P(\rho, \delta)=\rho^{2} \frac{\partial}{\partial \rho} e(\rho, \delta) .
$$

Here, $\rho$ and $\delta$ are the baryon density and the relative neutron excess, respectively. In most models the compressibility $K$ decreases as the matter becomes more neutronrich. But the rate of decrease varies widely among models, in particular at high densities.

Fig. 2.3 shows the results from a non-relativistic Hartree-Fock calculation [35]. The upper and lower windows are obtained using the density-dependent Reid and Paris nucleon-nucleon interactions, respectively. One finds from these studies that neutrons tend to make the nuclear EOS stiffer. Also, at densities above about $1.5 \rho_{0}$ the predicted density dependence of nuclear compressibility depends sensitively on the isospin independent part of the nucleon-nucleon interaction [35. This is because the strength of isospin-dependent interaction is generally much weaker than the isospinindependent one. The saturation density at each relative neutron excess $\delta$ is indicated by the solid dot in Fig. 2.3 and is seen to shift towards a lower density as the nuclear matter becomes more neutron-rich. A similar decrease is seen for the nuclear compressibility at saturation density as a result of its strong density dependence.

A non-relativistic study of the isospin dependence of the nuclear compressibility and density at saturation has been carried out by Kolehmainen et al. [36] using the Skyrme-type interaction in an extended Thomas-Fermi approach. Their prediction 


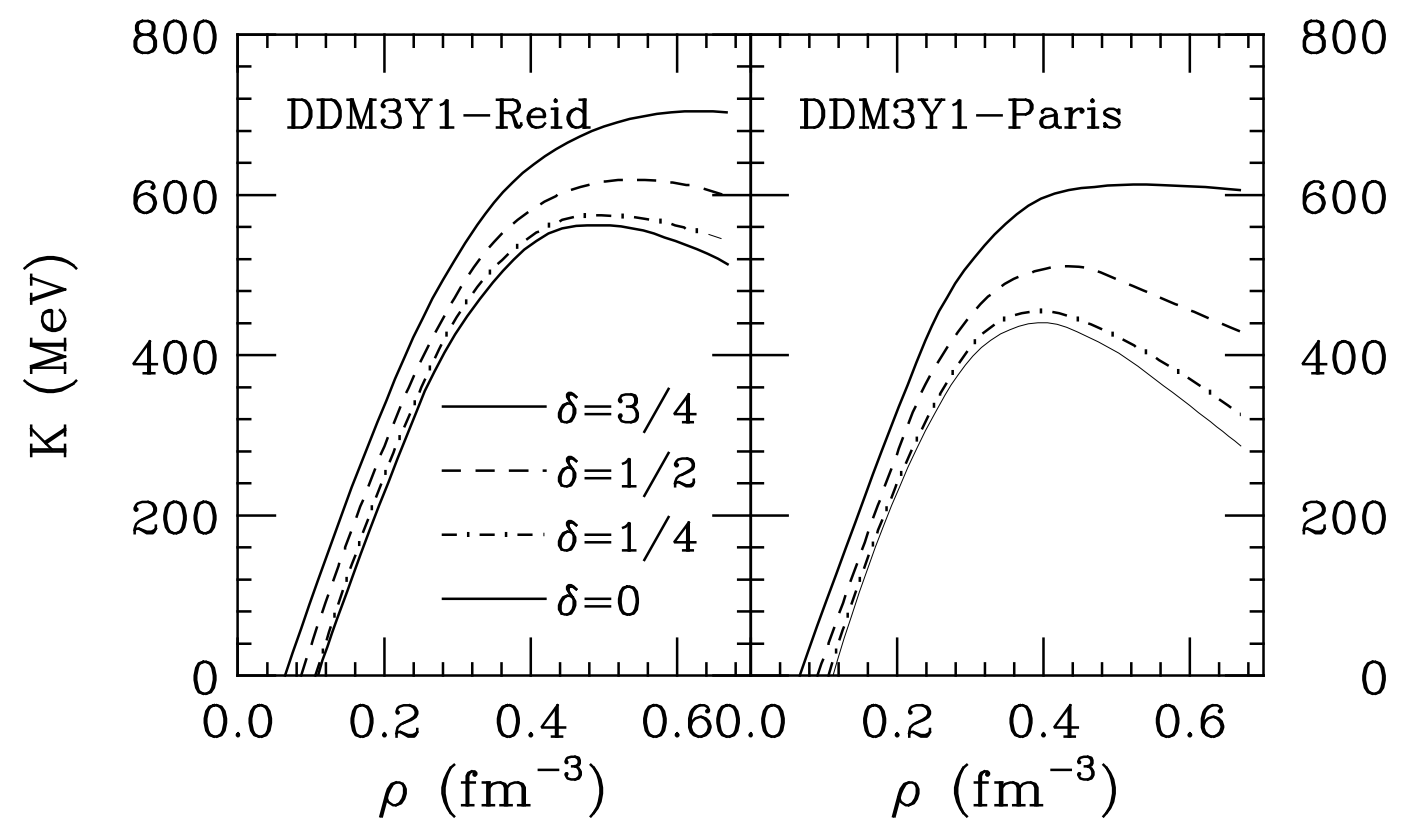

Figure 2.3: The nuclear compressibility $K$ obtained from the non-relativistic HartreeFock calculations using the density-dependent Reid (left window) and Paris (right window) nucleon-nucleon interactions, respectively. Results taken from Ref. [35].

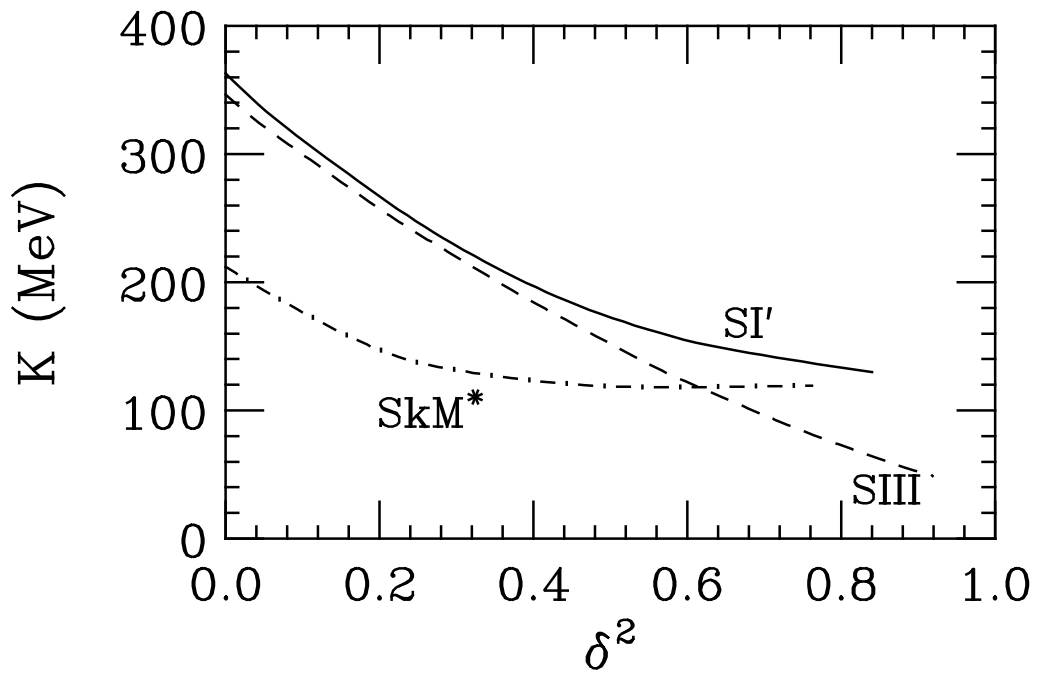

Figure 2.4: Nuclear compressibility as a function of relative neutron excess, obtained from the extended Thomas-Fermi calculations using three different Skyrme interactions. Results taken from Ref. [36]. 
Table 2.1: Parameters $K_{0}$ and $a$ for the isospin dependence of the compressibility at saturation.

\begin{tabular}{cccccccc}
\hline Force & Paris & SKM $^{*}$ & SI $^{\prime}$ & SIII & AV14+UVII & UV14+UVII & FP \\
\hline$K_{0}(0)$ & 185 & 216.6 & 370.3 & 355.3 & 209 & 202 & 238 \\
$a$ & 2.027 & 1.988 & 1.272 & 1.275 & 2.196 & 2.049 & 1.01 \\
$K_{0}\left(\frac{1}{3}\right)$ & 143.3 & 168.7 & 318.0 & 305.0 & 158 & 156 & 212 \\
\hline
\end{tabular}

on the isospin dependence of the nuclear compressibility at saturation $K_{0}(\delta)$ is shown in Fig. 2.4, and their results can be parameterized as a quadratic function of $\delta$

$$
K_{0}(\delta)=K_{0}(0)\left(1-a \delta^{2}\right)
$$

For small $\delta$ this parameterization works well and has been confirmed by other studies. For example, Bombaci and Lombardo 20 obtained a similar result using a non-relativistic Brueckner-Bethe-Goldstone approach with the Paris interaction. We summarize in Table 2.1 the parameters $K_{0}(0)$ in units of $\mathrm{MeV}$ and $a$ extracted from these studies [20, 25, 36] and those from fitting the EOs calculated by Wiringa et al. [27] using the variational many-body (VMB) approach with the Argonne and Urbana two-body potentials AV14 and UV14 augmented by the Urbana three-body potential UVII, as well as that from Friedman and Pandharipande (FP) 25 are also listed. As one expects, both $K_{0}(0)$ and $a$ extracted from different models or the same model but different nuclear interactions vary appreciably.

We also show in Table 2.1 the compressibility $K_{0}\left(\frac{1}{3}\right)$ at $\delta=1 / 3$, which is about $20-30 \%$ below $K_{0}(0)$ and is of special interest in nuclear astrophysics. According to the prompt explosion model of supernova [9], a neutron-rich system is formed during the collapse of massive stars as a result of neutrino trapping, and the isospin asymmetry $\delta$ of a star stays almost at a constant value of $1 / 3$. In this study, Eq. (2.4) with the parameter set of $\mathrm{SKM}^{*}$ was used, and it was found that the softening of nuclear EOs due to the isospin asymmetry is responsible for generating a successful, prompt explosion. To our best knowledge, this is probably the only astrophysical evidence that supports the predicted isospin dependence of nuclear compressibility at saturation. In laboratory experiments, a 30\% reduction in compressibility seems too small to have a measurable effect even on nuclear collective flow which is believed to be the most sensitive probe to the compressibility. However, the corresponding changes in the mean-field potentials for neutrons and protons are opposite in sign, and this makes certain observables, such as the neutron to proton ratio of preequilibrium nucleons in heavy-ion collisions, sensitive to the isospin dependence of the EOS. We shall discuss this in more detail in section 4.2. 


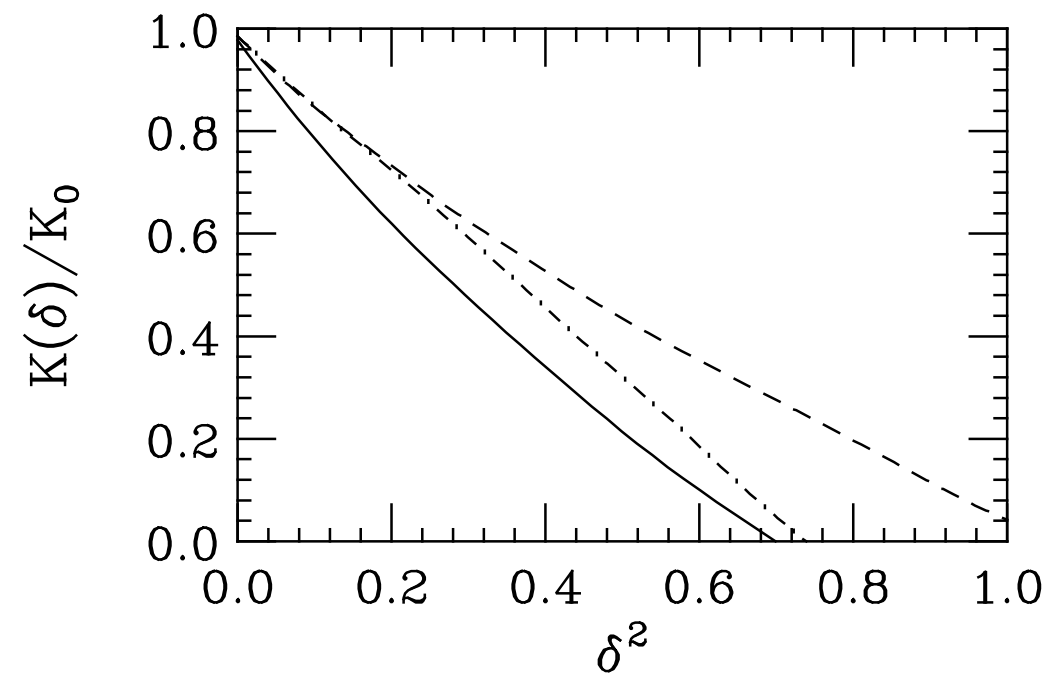

Figure 2.5: Relative compressibility at saturation obtained from the Dirac-HartreeFock calculations using isoscalar mesons only (dashed line), both isoscalar and isovector (solid line), and the non-relativistic Hartree-Fock calculations using the Skyrme SIII interaction (dot-dashed line). Results taken from Ref. [33].

There are also many calculations of the nuclear compressibility using a relativistic approach. Although it is not easy to compare different model calculations as they usually involve many parameters and assumptions, the comparison made in Ref. [33] is very instructive. Shown in Fig. 2.5 is a comparison of the isospin dependence of the ratio $K_{0}(\delta) / K_{0}(0)$ calculated in the framework of a Dirac-Hartree-Fock approach using only isoscalar $(\sigma, \omega)$ mesons (curve b) and both isoscalar and isovector $(\pi, \rho)$ mesons (curve e) as well as from the non-relativistic Hartree-Fock approach using the Skyrme SIII interaction (curve SIII). The general trend of decreasing compressibility with increasing isospin asymmetry $\delta$ is common to all three models. The rate of decrease is, however, sensitive to the details in the model.

Other properties at saturation, such as the binding energy, pressure and density, also show dependence on the isospin asymmetry $\delta$ of nuclear matter. Shown in Fig. 2.6 are the binding energy and density at saturation as functions of $\delta^{2}$ [33]. As for the compressibility at saturation, the saturation density can be parameterized as

$$
\rho_{e q}(\delta)=\rho_{0}(0)\left(1-b \delta^{2}\right),
$$

In Table 2.2 we summarize the values of the parameters $b$ and $\rho_{0}(0)$ extracted from several models. Results from the Dirac-Hartree-Fock approach are denoted by DHF (b) and DHF (e) for using isoscalar mesons only and both isoscalar and isovector mesons, respectively. From tables 2.2 and 2.1 one can clearly see a large variation of the parameters describing the isospin dependence of the compressibility and saturation 


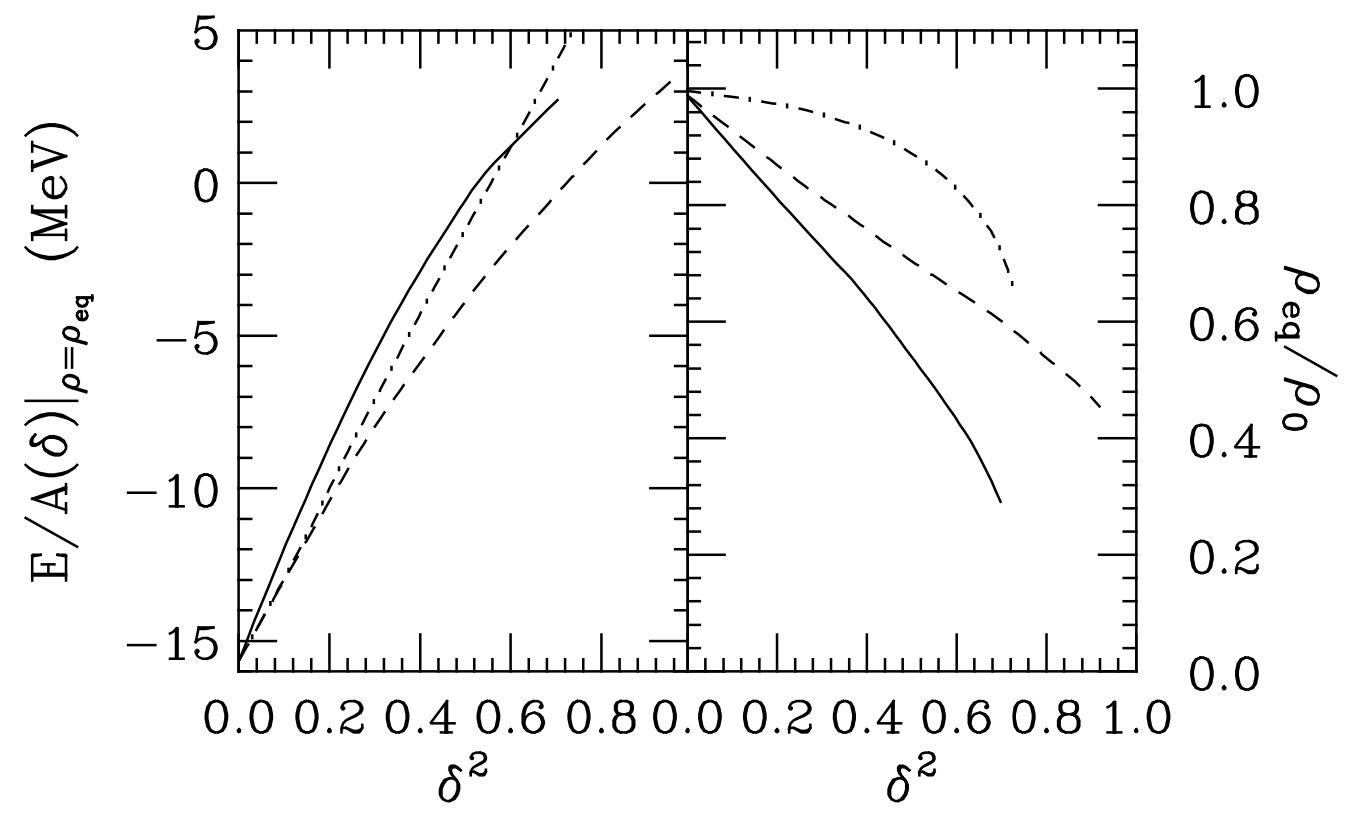

Figure 2.6: Same as Fig. 2.5 for the energy (left) and density (right) at saturation. Results taken from Ref. [33].

density. Obviously, experimental data are needed to test these models and thus to narrow down the isospin-dependence of the nuclear EOS. In this respect, collisions of neutron-rich or radioactive nuclei are promising tools. We shall come back to this point later.

Table 2.2: Parameters $\rho_{0}$ and $b$ in the isospin dependence of saturation density.

\begin{tabular}{ccccccc}
\hline Force & Paris & SKM $^{*}$ & SI $^{\prime}$ & SIII & DHF (b) & DHF (e) \\
\hline$\rho_{0}(0)$ & 0.289 & 0.1603 & 0.1553 & 0.1453 & 0.1484 & 0.1484 \\
$b$ & 1.115 & 0.634 & 0.286 & 0.084 & 0.65 & 0.9 \\
\hline
\end{tabular}




\subsection{Empirical parabolic law and the nuclear sym- metry energy}

For asymmetric nuclear matter at densities away the saturation density, various theoretical studies, e.g., Refs. 111, 17, 26, 27, 42, 43, 44, have shown that the energy per nucleon can be well approximated by

$$
e(\rho, \delta)=T_{F}(\rho, \delta)+V_{0}(\rho)+\delta^{2} V_{2}(\rho)
$$

where

$$
T_{F}(\rho, \delta)=\frac{3 \hbar^{2}}{20 m}\left(\frac{3 \pi^{2} \rho}{2}\right)^{2 / 3}\left[\delta^{5 / 3}+(1-\delta)^{5 / 3}\right]
$$

is the Fermi-gas kinetic energy, $V_{0}(\rho)$ and $V_{2}(\rho)$ are the isospin-independent and dependent potential energies. Higher-order terms in $\delta$ are negligible. For example, the magnitude of the $\delta^{4}$ term at $\rho_{0}$ has been estimated to be less than $1 \mathrm{MeV}$ [17, 18, 26]. Eq. (2.6) can be further approximated by

$$
e(\rho, \delta)=e(\rho, 0)+e_{\mathrm{sym}}(\rho) \delta^{2}
$$

where $e(\rho, 0)$ is the EOS of symmetric nuclear matter and

$$
e_{\mathrm{sym}}(\rho)=\left.\frac{1}{2} \frac{\partial^{2} e(\rho, \delta)}{\partial \delta^{2}}\right|_{\delta=0}=\frac{5}{9} T_{F}(\rho, 0)+V_{2}(\rho)
$$

is the bulk symmetry energy. At normal nuclear matter density, the two terms in the above equation have about similar magnitude. Eq. (2.8) is known as the empirical parabolic law for the EOS of asymmetric nuclear matter and is considered to be valid only at small isospin asymmetries. However, many non-relativistic and relativistic calculations have shown that it is actually valid up to $\delta=1$. In Fig. 2.7 and Fig. 2.8, two examples from the non-relativistic Brueckner-Bethe-Goldstone approach [20] and the relativistic-Brueckner-Hartree-Fock approach [24], respectively, are shown for the total binding energy as a function of $\delta^{2}$ at several densities $\rho$. In both cases, the fit using the parabolic law shown by solid lines is indeed found to be valid in the whole range of $\delta$.

Using the empirical parabolic law, one can easily extract the symmetry energy $e_{\text {sym }}(\rho)$ from microscopic calculations. According to Eq. (2.8) the bulk symmetry energy $e_{\text {sym }}(\rho)$ can be evaluated from the two extreme cases of both pure neutron matter and symmetric nuclear matter via

$$
e_{\mathrm{sym}}(\rho)=e(\rho, 1)-e(\rho, 0)
$$

Furthermore, the symmetry energy can be expanded around the normal nuclear matter density $\rho_{0}$, i.e.,

$$
e_{\mathrm{sym}}(\rho)=e_{\mathrm{sym}}\left(\rho_{0}\right)+\frac{L}{3}\left(\frac{\rho-\rho_{0}}{\rho_{0}}\right)+\frac{K_{\mathrm{sym}}}{18}\left(\frac{\rho-\rho_{0}}{\rho_{0}}\right)^{2}+\cdots .
$$




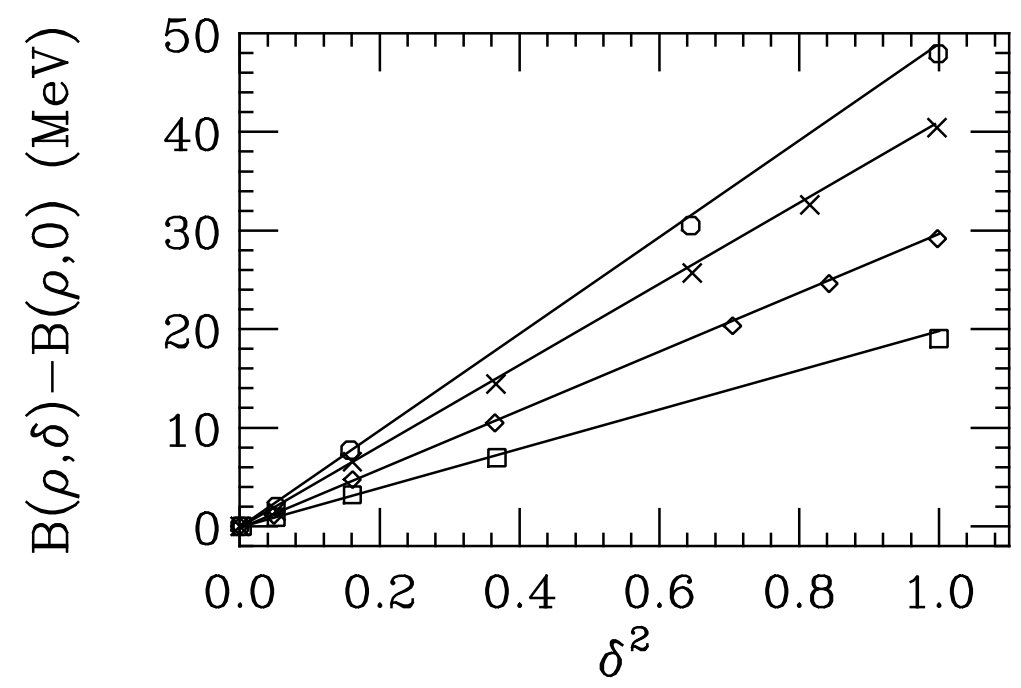

Figure 2.7: Total binding energy per nucleon as a function of $\delta^{2}$ for densities (from top to bottom) of $0.4,0.3,0.17$, and $0.076 \mathrm{fm}^{-3}$, obtained from the non-relativistic Brueckner-Bethe-Goldstone approach. Solid lines are the fits using the parabolic law to that from numerical calculations. Taken from Ref. [20].

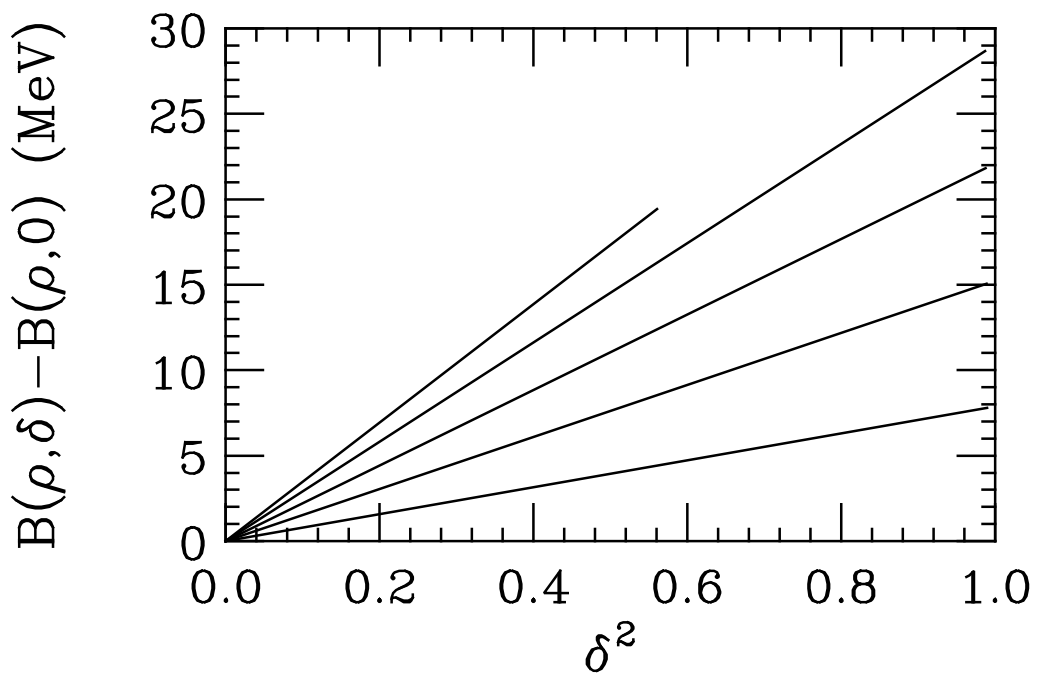

Figure 2.8: Same as Fig. 2.7 obtained from the relativistic Brueckner-Hartree-Fock approach. The lines correspond to values of the density of (from top to bottom) 0.18 , $0.14,0.1,0.06$, and $0.02 \mathrm{fm}^{-3}$. Results taken from Ref. 24. 
Table 2.3: The slope and curvature of symmetry energy in units of MeV at normal nuclear density.

\begin{tabular}{ccccccc}
\hline Force & Paris & SKM $^{*}$ & SI $^{\prime}$ & SIII & DHF (b) & DHF (e) \\
\hline$L$ & 68.8 & 45.78 & 35.34 & 9.91 & 132 & 138 \\
$K_{\text {sym }}$ & 37.56 & -155.9 & -259.1 & -393.7 & 466 & 276 \\
\hline
\end{tabular}

In the above, $L$ and $K_{\text {sym }}$ are the slope and curvature of the symmetry energy at normal density, i.e.,

$$
\begin{aligned}
L & \left.\equiv 3 \rho_{0} \frac{\partial e_{\text {sym }}}{\partial \rho}\right|_{\rho=\rho_{0}}, \\
K_{\text {sym }} & \left.\equiv 9 \rho_{0}^{2} \frac{\partial^{2} e_{\text {sym }}}{\partial^{2} \rho}\right|_{\rho=\rho_{0}} .
\end{aligned}
$$

With this expansion, the predicted symmetry energy at normal nuclear matter density can be compared with that determined from the mass formula, which gives a value of $e_{\text {sym }}\left(\rho_{0}\right)$ in the range of $27-36 \mathrm{MeV}$ 45]. Most models are tuned to give an $e_{\text {sym }}\left(\rho_{0}\right)$ within this range. For example, in the non-relativistic Hartree-Fock theory [46, 47], the predicted value of $e_{\text {sym }}\left(\rho_{0}\right)$ is between 27 and $38 \mathrm{MeV}$ depending on the nuclear interactions used in the calculation. However, the relativistic mean-field (RMF ) theory 28, 32, 48, 49, 50, 51 usually gives higher values of $e_{\text {sym }}\left(\rho_{0}\right)$ in the range of 35-42 MeV. What distinguishes these models around normal nuclear matter density are thus the slope $L$ and curvature $K_{\text {sym }}$. This is clearly illustrated in Fig. 2.9 where results from several non-relativistic approaches [20, 19, 27 are compared. Although all predict a $e_{\text {sym }}\left(\rho_{0}\right)$ in the range of $27-30 \mathrm{MeV}$ (in agreement with that from the empirical mass formula), it is seen that the slope and curvature at $\rho_{0}$ are very different. As shown in Table $2.3, K_{\text {sym }}$ ranges from positive $466 \mathrm{MeV}$ to negative $400 \mathrm{MeV}$. The most recent data from giant monopole resonances indicate that $K_{\text {sym }}$ is between $-566 \pm 1350 \mathrm{MeV}$ to $34 \pm 159 \mathrm{MeV}$ [52] depending on the mass region of nuclei and the number of parameters used in parametrizing the compressibility of finite nuclei. The $K_{\text {sym }}$ data thus still cannot distinguish these model calculations.

In radioactive nuclei as well as neutron stars, the symmetry energy at densities away from the normal nuclear matter saturation density is relevant. Although the Bethe-Goldstone theory predicts a $\rho^{1 / 3}$ dependence for the symmetric energy, the density dependence is linear in the RMF theory [28, 29, 53]. Results from most other calculations cannot be simply described. In the most sophisticated calculations by Wiringa et al. using the variational many-body (vMB) theory [27], the density dependence of $e_{\mathrm{sym}}(\rho)$ has been calculated using either the Argonne two-body potential 


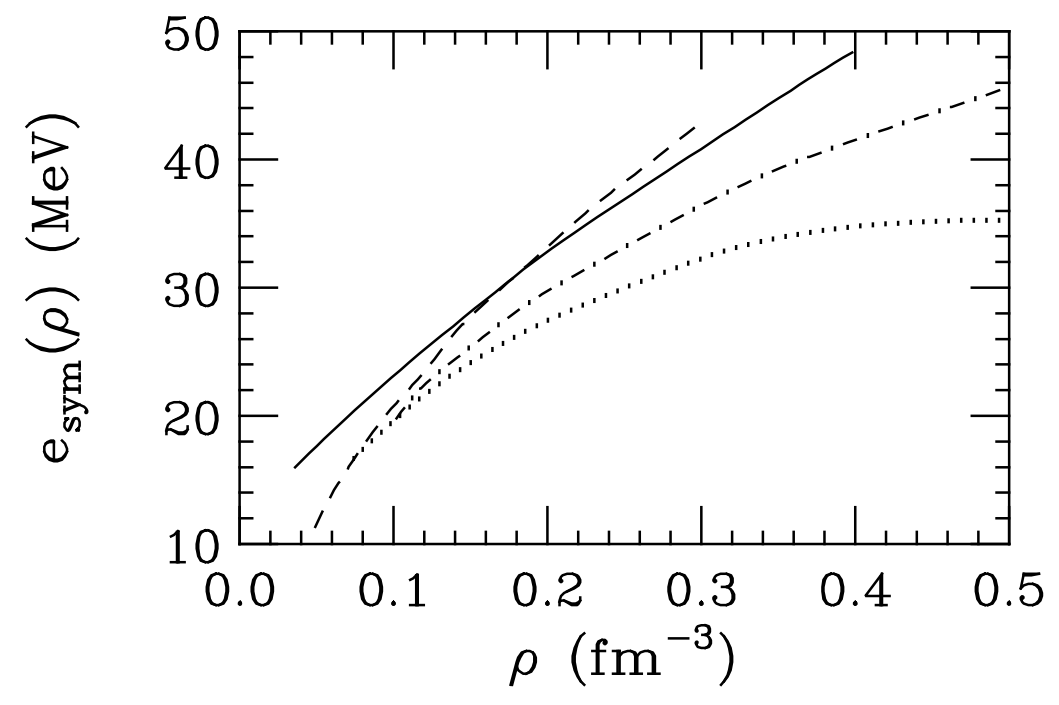

Figure 2.9: A comparison of the density dependence of the symmetry energy predicted by several non-relativistic many-body calculations: Paris (solid line), AV14 (dotted line), uf14 (dot-dashed line), and Paris+3BF (dashed line). Results taken from ref. [19, 20, 27].

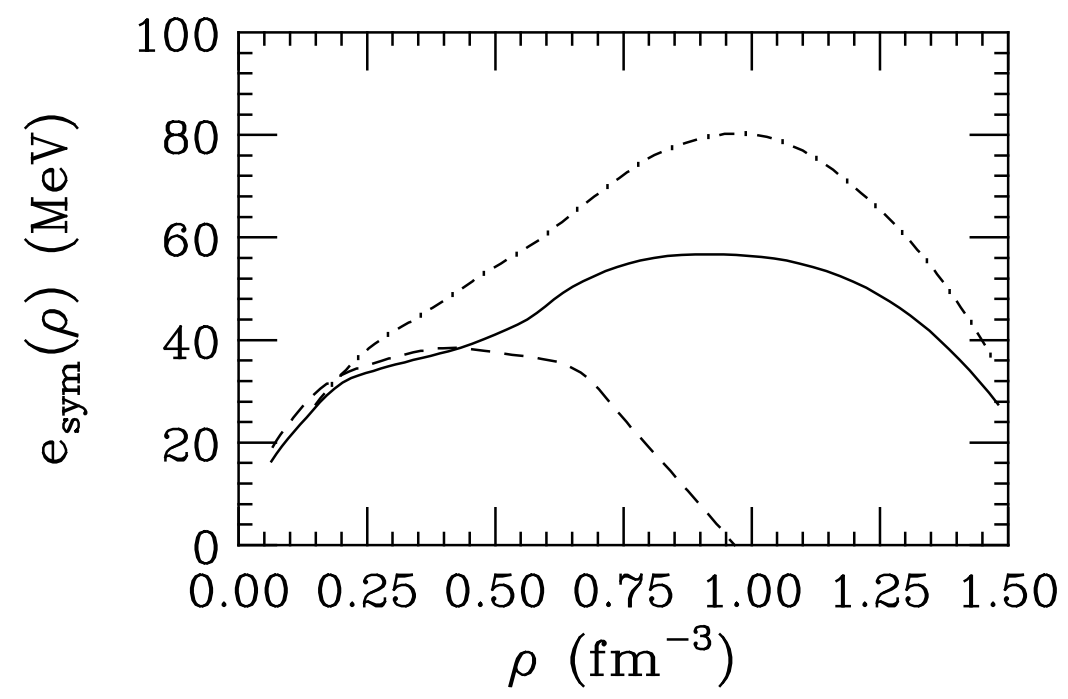

Figure 2.10: Symmetry energy predicted by variational many-body calculations: AV14+UVII (solid line), Uv14+UVII (dot-dashed), and Uv14+TNI (dashed). Results taken from Ref. [27]. 
AV14 or Urbana UV14 together with either the three-body potential UVII or TNI, and the results differ appreciably as shown in Fig. 2.10. With three-body interactions the symmetry energy is seen to vanish at high densities. Therefore, pure neutron matter was predicted by the VMB theory as the ground state of dense nuclear matter. This prediction has important consequences on the structure and magnetic properties of neutron stars as stressed recently by Kutschera et al. 54. However, other models predict a nonzero symmetry energy at high densities. Among all models, the pre-

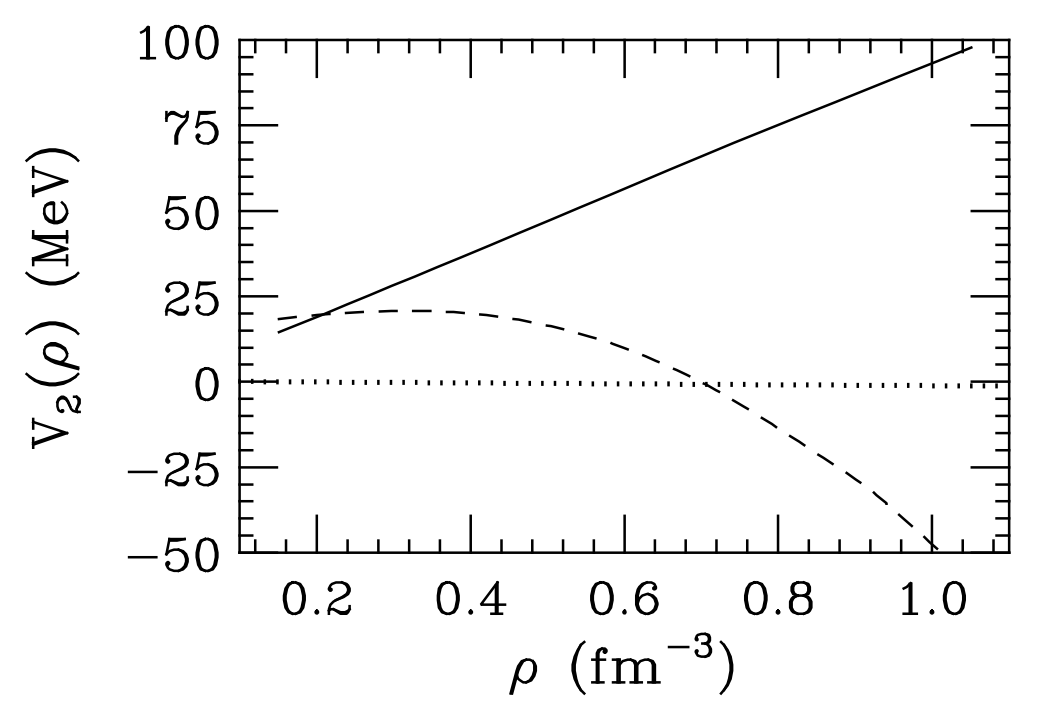

Figure 2.11: Contribution from nuclear interactions to the symmetry energy in the relativistic mean field (RMF) theory (solid line) and the variational many-body (VMB) theory (dashed line). Results taken from Ref. [54].

dictions from the RMF theory and the VMB theory using the UV14+TNI interaction differ the most. Since the kinetic contribution to the symmetry energy is about the same in all model calculations, the differences among model predictions are mainly from the $V_{2}(\rho)$ term in Eq. (2.9). As shown in Fig. 2.11 the $V_{2}(\rho)$ term is always repulsive and increases linearly with density in the RMF theory, while in the VMB theory it changes from repulsion to attraction as the density increases. The variation of $V_{2}(\rho)$ with density in the VMB theory can be understood in terms of the behavior of nuclear interactions in dense nuclear matter [55]. At high densities the short-range repulsion dominates and is greater for a nucleon pair in an isospin singlet $(T=0)$ than in an isospin triplet $(T=1)$ state. Pure neutron matter is therefore more stable. At moderate densities the strong attractive isospin singlet tensor potential and correlations keep the isospin singlet pairs more bound, and symmetric nuclear matter is thus more stable than pure neutron matter. These features do not exist in the RMF theory where the asymmetry energy is due to the rho meson exchange, which leads to a repulsive $V_{2}(\rho)$ at all densities. As a result, these two approaches predict very 
different structures and chemical compositions for neutron stars [54].

It is clear from the widely diverging model predictions that despite great theoretical efforts our knowledge of the EOS of asymmetric nuclear matter is rather limited. In particular, the behaviour of the symmetry energy at high densities is most uncertain among all properties of dense nuclear matter [54].

\subsection{Neutron stars and the EOS of asymmetric nu- clear matter}

The mechanism for supernova explosion and the properties of neutron stars have been subjects of much interest; for recent reviews, see e.g., Refs. [56, 57, 58, 59, 60]. Here we only concentrate on the effects of the isospin-dependence of the EOS, in particular the density dependence of symmetry energy, on the properties of neutron stars. Various studies have indicated that the symmetry energy affects mainly the chemical composition of neutron stars [11, 21, 38, 43, 61, 62]. Other properties, such as the cooling rate, lepton profiles and neutrino flux, which depend on the chemical composition, will therefore also be affected.

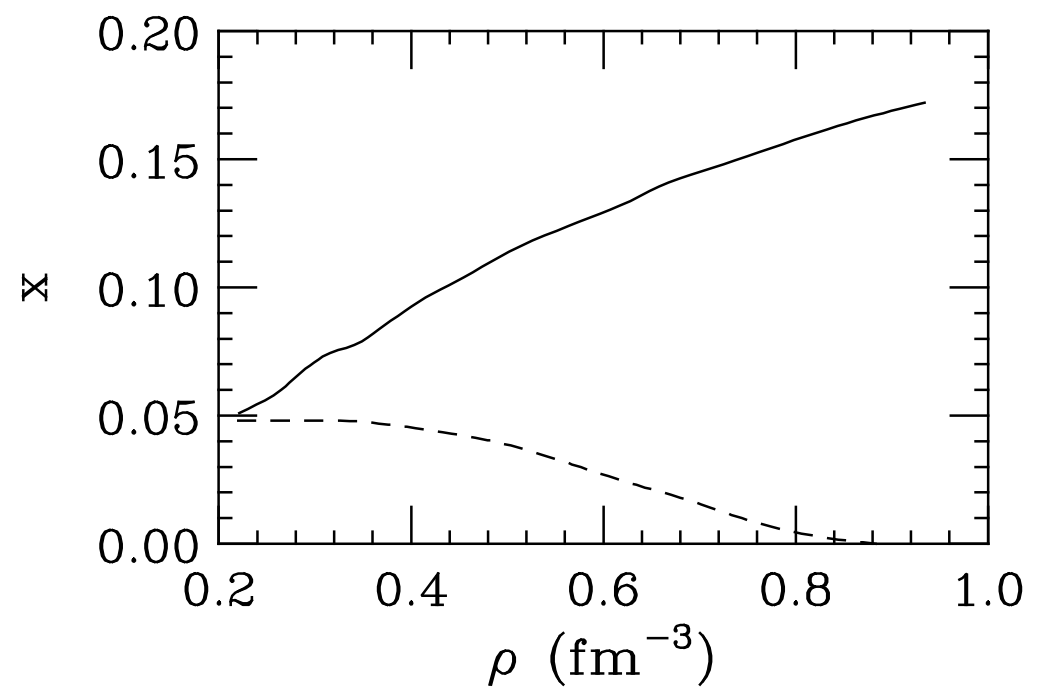

Figure 2.12: Equilibrium fraction of protons in neutron stars predicted by the relativistic mean-field (RMF, solid line) and variational many-body (VMB, dashed line) theories. Taken from Ref. [54].

In the most simple picture, a neutron star is composed of neutrons, protons and electrons with a proton fraction of

$$
x=\frac{1}{2}(1-\delta) .
$$


The condition for $\beta$-equilibrium in terms of the chemical potentials of electrons $\left(\mu_{e}\right)$, neutrons $\left(\mu_{n}\right)$ and protons $\left(\mu_{p}\right)$ is

$$
\mu_{e}=\mu_{n}-\mu_{p}=-\frac{\partial e(\rho, \delta)}{\partial x}=4 e_{\mathrm{sym}}(\rho)(1-2 x) .
$$

For relativistic degenerate electrons of density $\rho_{e}=\rho_{p}=x \rho$, charge neutrality requires

$$
\mu_{e}=\left(m_{e}^{2}+p_{F_{e}}^{2}\right)^{1 / 2} \approx \hbar c\left(3 \pi^{2} \rho x\right)^{1 / 3},
$$

which together with Eqs. (2.15) and (2.8) determine an equilibrium proton fraction $x$ given by

$$
\hbar c\left(3 \pi^{2} \rho x\right)^{1 / 3}=4 e_{\text {sym }}(\rho)(1-2 x) .
$$

The equilibrium proton fraction $x$ is therefore determined solely by the nuclear symmetry energy, $e_{\mathrm{sym}}(\rho)$. At high densities such that $\mu_{e} \geq m_{\mu}$ both electrons and muons are present at $\beta$-equilibrium. The inclusion of muons mainly alters the value of the equilibrium proton fraction, $x$, but not its density dependence. Therefore, the difference in $x$ predicted by using different symmetry energies is about the same with or without including muons [11, 27]. As discussed in the previous section, the RMF and the VMB theory with the AV14+TNI interaction differ most in their predictions on the symmetry energy at high densities. Their predicted equilibrium proton fractions at high densities are therefore also very different as shown in Fig. 2.12. We see that the RMF theory predicts a linear increase, while in the VMB theory the proton fraction in neutron stars gradually decreases as the density increases. The disappearance of protons in neutron stars is a common feature of the VMB theory, although the critical density at which this occurs depends on the interaction used in the calculation. As a result, there exists an instability in neutron stars with respect to the separation of protons and neutrons at low densities. This has thus led to the suggestion that polarons, which are localized protons surrounded by neutron bubbles, can be formed in neutron stars [54]. In Fig. 2.13 we compare the equilibrium proton fractions (lower window) corresponding to different symmetry energies (upper window)

$$
e_{\mathrm{sym}}(\rho)=\left(2^{2 / 3}-1\right) \frac{3}{5} E_{F}^{0}\left[u^{2 / 3}-F(u)\right]+e_{\mathrm{sym}}\left(\rho_{N M}\right) F(u),
$$

with $F(u)$ having one of the following three forms

$$
\begin{aligned}
& F_{1}(u)=\frac{2 u^{2}}{1+u}, \\
& F_{2}(u)=u, \\
& F_{3}(u)=u^{1 / 2},
\end{aligned}
$$

where $u \equiv \rho / \rho_{0}$ is the reduced baryon density and $E_{F}^{0}$ is the Fermi energy. These forms of the symmetry energy resemble closely the three typical results from microscopic many-body calculations discussed in the previous section. Although in all three cases $x$ increases with density, the differences among them are still appreciable. 


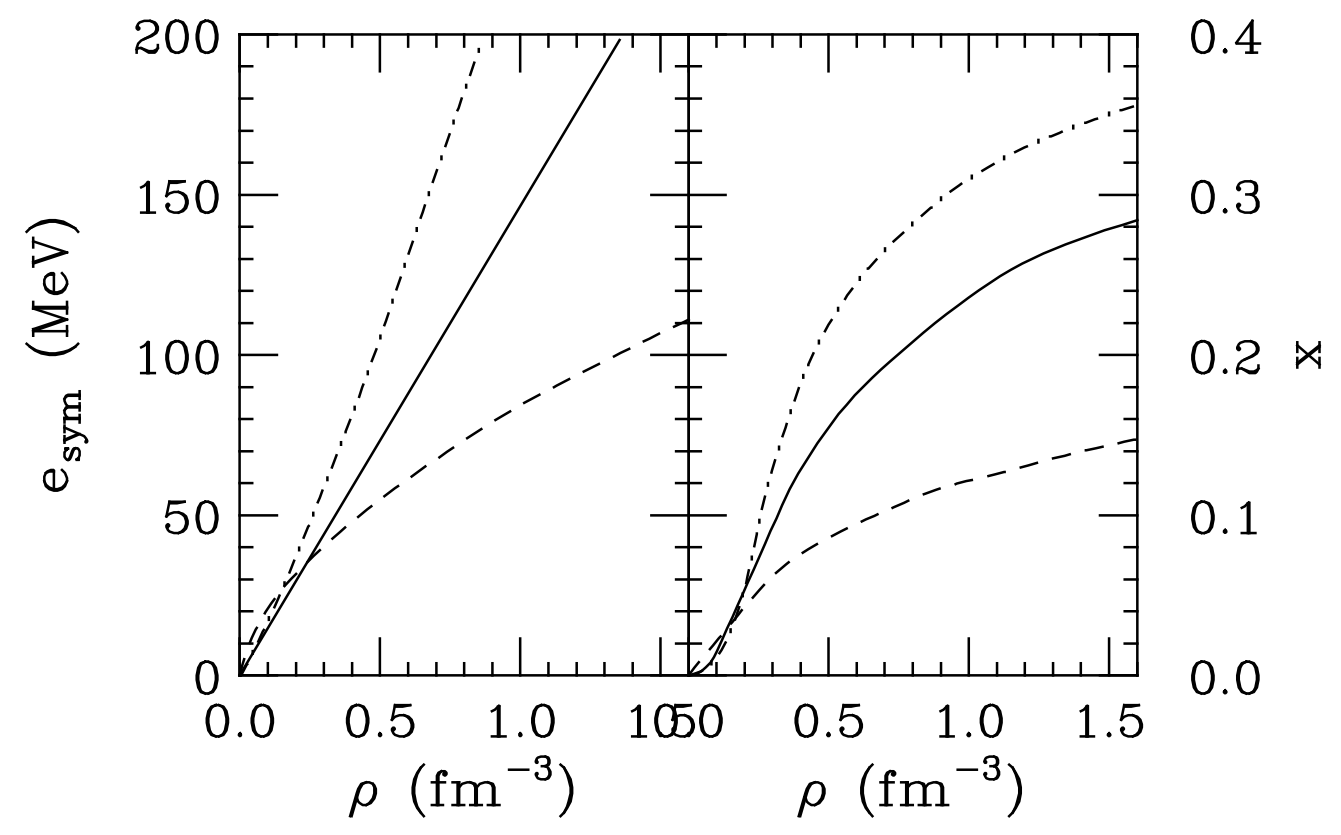

Figure 2.13: Left window: symmetry energy as a function of density. Right window: corresponding equilibrium fraction of protons. Solid lines: $F_{2}(u)$, dashed lines: $F_{3}(u)$, dash-dotted lines: $F_{1}(u)$. Results taken from Ref. [11].

The variation in symmetry energy, especially at high densities, results in a corresponding variation in the proton fraction $x$. This has significant effects on the cooling of neutron stars and the kaon condensation in dense stellar matter. In the so-called standard model for neutron stars, their cooling is mainly due to the modified URCA process

$$
(n, p)+p+e^{-} \rightarrow(n, p)+n+\nu_{e}, \quad(n, p)+n \rightarrow(n, p)+p+e^{-}+\overline{\nu_{e}} .
$$

The direct URCA process

$$
n \rightarrow p+e^{-}+\overline{\nu_{e}}, \quad p+e^{-} \rightarrow n+\nu_{e}
$$

is usually forbidden by energy-momentum conservation. However, it has been shown by Lattimer et al. [11] that if the proton fraction is higher than a critical value of about $1 / 9$, the direct URCA process can occur. This would then enhance the emission of neutrinos; so the neutron star cooling rate is increased significantly. From Fig. 2.13 it is seen that the symmetry energy determines entirely whether the proton fraction can exceeds the critical value and at what density this happens.

Another important effect of the symmetry potential is on the kaon condensation in dense stellar matter. Since electrons with chemical potentials higher than the kaon mass will trigger the process $e^{-} \rightarrow K^{-} \nu_{e}$, the condition for this to happen is determined by the equation $\mu_{e} \geq m_{k^{-}}$. It was shown in ref. [13] that the critical density for kaon condensation depends sensitively on the form of the symmetry potential. 


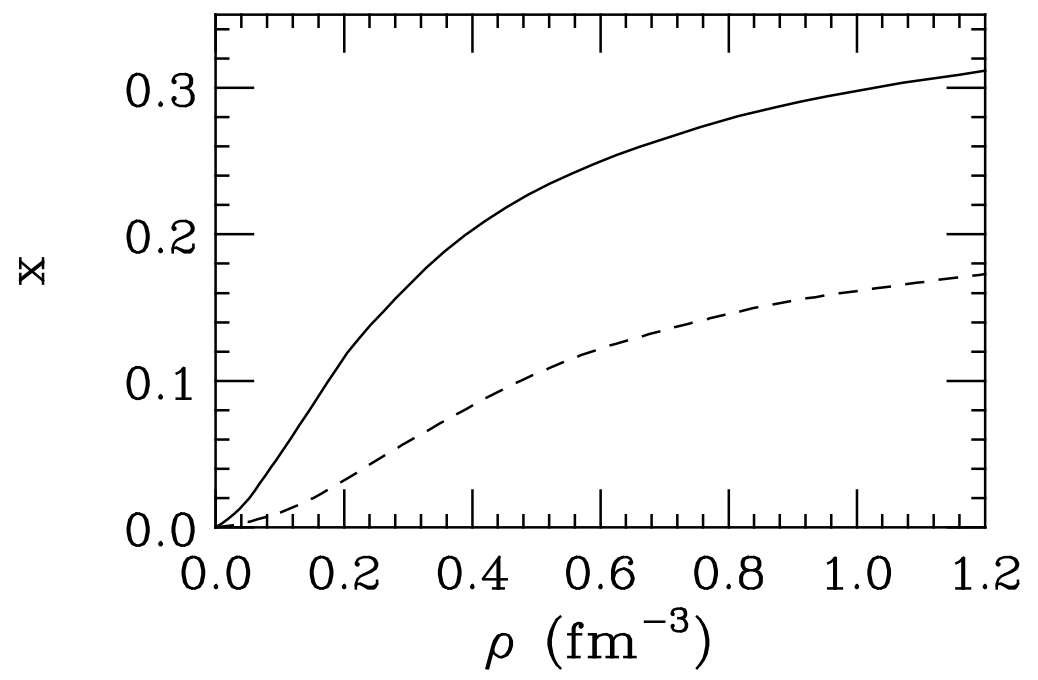

Figure 2.14: Equilibrium fraction of protons as a function of density obtained from the relativistic mean field theory. Solid curve: $g_{p}=5.507$, dashed curve: $g_{p}=2.78$. Results taken from Ref. [23].

The dependence of equilibrium proton fraction on the underlying nuclear interaction can be illustrated by the recent work of Sumiyoshi et al. using the relativistic mean-field (RMF) theory [23, 61]. The symmetry energy is found to vary almost linearly with density, and its strength is related to the $\rho$ meson-nucleon coupling constant $g_{\rho}$ via

$$
e_{\mathrm{sym}}\left(\rho_{0}\right)=\frac{k_{F}^{2}}{6 \sqrt{M^{* 2}+k_{F}^{2}}}+g_{\rho}^{2} \rho_{0} / 2 m_{\rho}^{2},
$$

where $M^{*}$ is the nucleon effective mass, $m_{\rho}$ is the $\rho$ meson mass, and $k_{F}$ is the Fermi momentum. The first and second terms are the kinetic and potential contributions to the symmetry energy, respectively. From the above expression and Eq. (2.17), one obtains the $g_{\rho}$ dependence of the proton fraction as shown in Fig. 2.14. As $g_{\rho}$ increases from 2.78 to 5.507 the proton fraction is seen to increase by about a factor of two.

Eq. (2.22) also shows that the effective mass of a nucleon, $M^{*}$, affects the kinetic contribution to the symmetry energy. As nuclear matter becomes heated, which is expected in neutron stars, the nucleon effective mass may change. Donati et al. 63. have recently studied the coupling of the mean-field single-particle motion to the surface vibration within the quasiparticle random-phase approximation and found that $M^{*}$ decreases with increasing temperature for $T \leq 2 \mathrm{MeV}$, which are the temperatures relevant for the pre-supernova collapse of massive stars. The corresponding increase of the symmetry energy was estimated to be about $2.5 \mathrm{MeV} /$ nucleon, which 
would then increase the shock energy by about half of the observed explosion energy of SN1987a. The outcome of the explosion from detailed numerical simulations thus depends sensitively on the symmetry energy. However, more recent studies of Dean et al. [64, using the Monte Carlo shell model and taking into account pairing correlations, indicate that the symmetry energy changes by less than $0.6 \mathrm{MeV}$ at the neutron star temperature. The temperature dependence of the nuclear symmetry energy thus remains controversial.

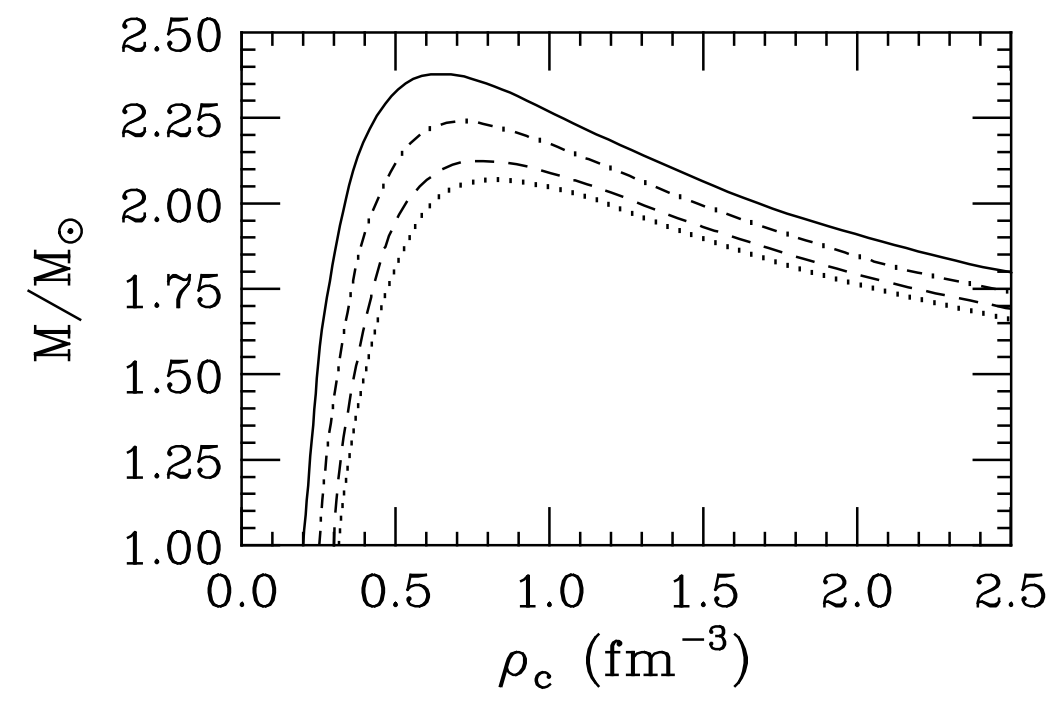

Figure 2.15: Mass of a neutron star $M / M_{\odot}$ (in solar mass units) as a function of its central density for various proton fractions, $x$. Solid line: $x=0$, dot-dashed: $x=0.15$, dashed: $x=0.3$, dotted: $x=0.45$. Results taken from Ref. [65].

The structure or density profile of a neutron star with an isotropic mass distribution can be determined by solving the well-known Tolman-Oppenheimer-Volkov equation, i.e.,

$$
\frac{d P}{d r}=-\frac{[\rho(r)+P(r)]\left[M(r)+4 \pi r^{3} P(r)\right]}{r^{2}-2 r M(r)},
$$

where $P(r)$ is the pressure obtained from the EOS via Eq. (2.3) and $M(r)$ is the gravitational mass inside a radius $r$. In Fig. 2.15 and Fig. 2.16 the mass and radius of a neutron star from a relativistic Brueckner Hartree-Fock approach are shown as a function of central density for various proton fractions [65]. A maximum mass of about $2.4 M_{\odot}$ and a radius of about $12 \mathrm{~km}$ is obtained, and this is rather large compared to the observed value of about $1.9 M_{\odot}$, indicating that the underlying EOS is too stiff. Pion and kaon condensates as well as effects of hyperons have been suggested as possible mechanisms to soften the EOS [65]. Large variations of both 


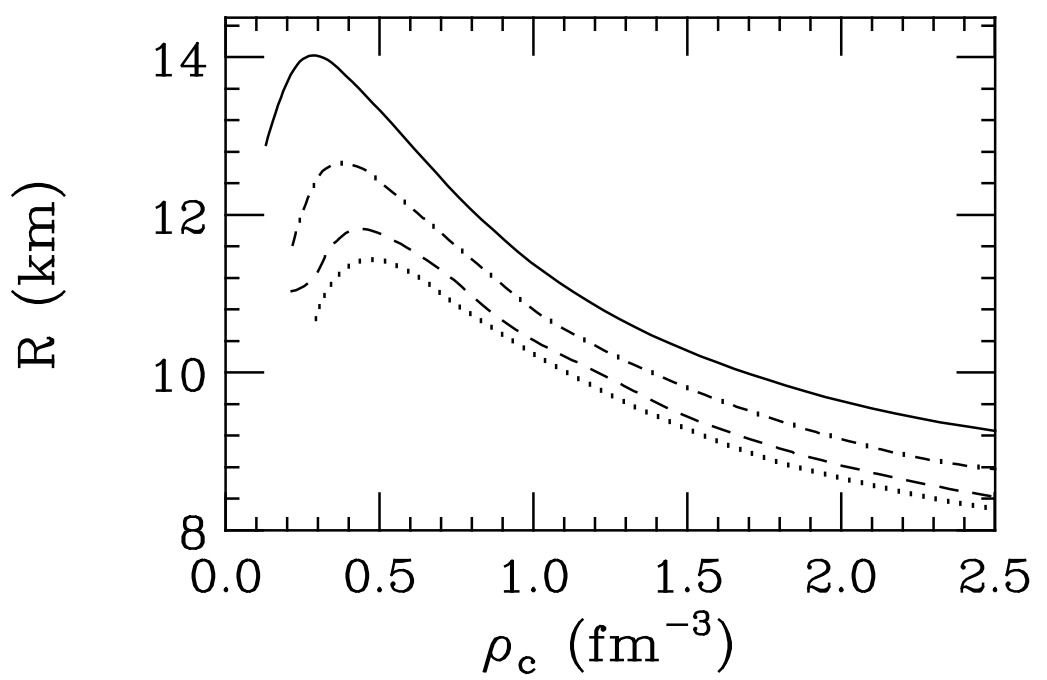

Figure 2.16: Radius of a neutron star as a function of its central density for various proton fractions. Solid line: $x=0$, dot-dashed: $x=0.15$, dashed: $x=0.3$, dotted: $x=0.45$. Results taken from Ref. 65.].

neutron star mass and density profile are observed by varying the proton fraction. However, the structure of a neutron star is indirectly affected by the symmetry energy through the equilibrium proton fraction. As shown in Refs. [43, 61, 66], the neutron star structure changes only slightly by varying $F(u)$ or $g_{\rho}$ in the ranges discussed above, and the stiffer the EOS is the less important is the symmetry energy. This is mainly because the symmetry energy is much smaller compared to the energy per nucleon in symmetric nuclear matter. Nevertheless, as we have mentioned earlier, the stiffness of the nuclear EOS is reduced by the neutrons. This reduction is important for generating a successful, prompt supernova explosion [9].

\subsection{Radioactive nuclei and the EOS of asymmetric nuclear matter}

The radius, thickness of neutron skins, deformation, binding energy, density distributions, and other properties of radioactive nuclei near the drip lines depend sensitively on the isospin-dependence of the nuclear EOS. Tanihata has recently stressed the possibility of extracting the EOS of asymmetric nuclear matter through the investigation of these properties [40]. In this review we discuss a complementary approach in extracting the isospin-dependent EOS via reactions of neutron-rich nuclei at intermediate energies. We shall first discuss the dependence of the properties of radioactive nuclei on the isospin-dependent EOS and then demonstrate how these properties are 
sensitive to the symmetry energy used in transport models for studying the dynamics of heavy-ion collisions.

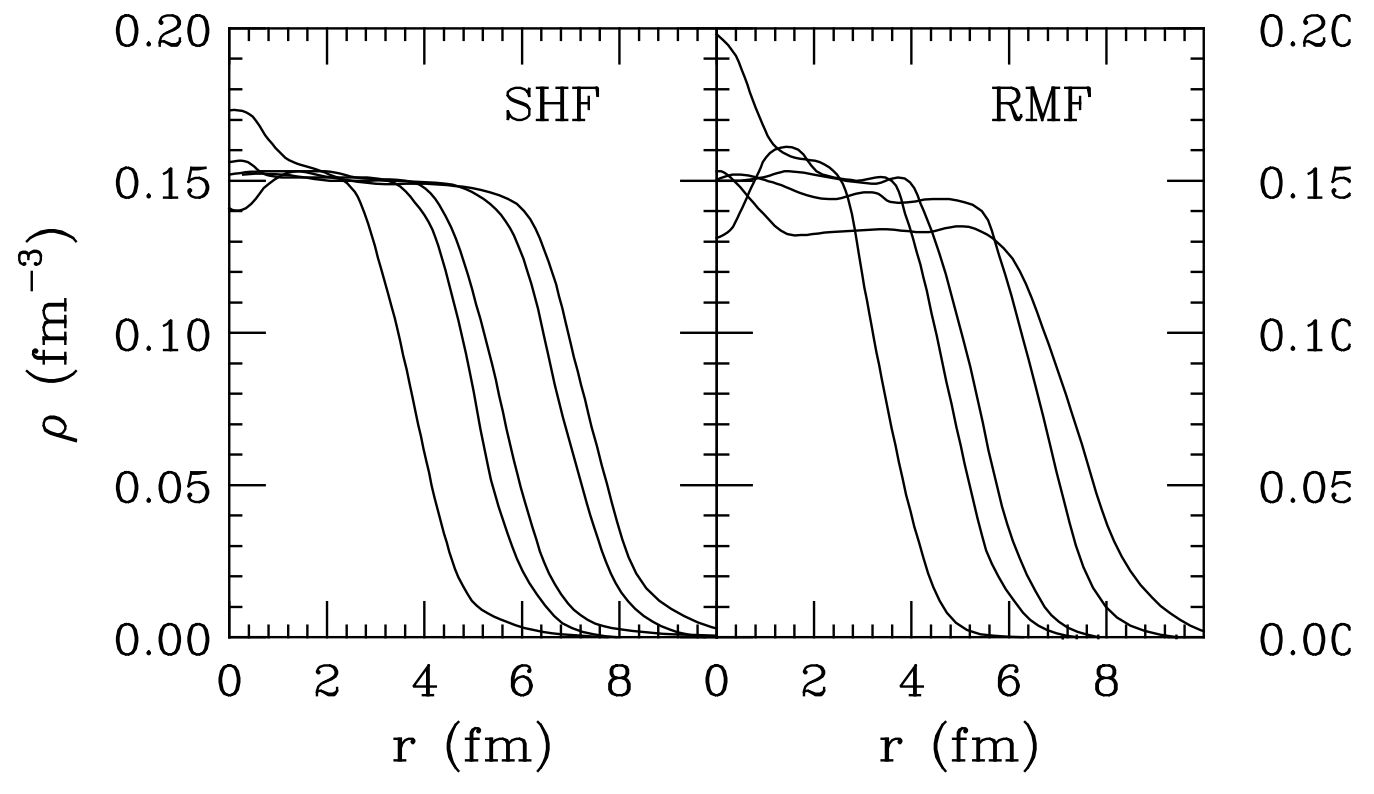

Figure 2.17: Density distributions for the nuclei ${ }^{266} \mathrm{~Pb},{ }^{208} \mathrm{~Pb},{ }^{120} \mathrm{Sn},{ }^{90} \mathrm{Zr}$, and ${ }^{40} \mathrm{Ca}$ as predicted by the Skyrme-Hartree-Fock (left) and the Relativistic Mean-Field (right) model. Results taken from Ref. [40].

In Fig. 2.1 the predictions on the EOs from the Skyrme-Hartree-Fock and the relativistic mean-field theories are compared. The properties of neutron rich nuclei have also been studied using these models. In Fig. 2.17, density distributions predicted by the two models for several nuclei are shown 40. A comparison of the results for ${ }^{208} \mathrm{~Pb}$ and ${ }^{266} \mathrm{~Pb}$ is particularly interesting. One sees that, although the central density from SHF does not change from ${ }^{208} \mathrm{~Pb}$ to ${ }^{266} \mathrm{~Pb}$, it decreases in RMF. This is related to the fact that in SHF the saturation density essentially does not change, but in RMF it decreases rapidly as nuclear matter becomes more neutron-rich as shown by the thick dashed lines in Fig. 2.1. As a result, the RMF gives a more prominent neutron skin for ${ }^{266} \mathrm{~Pb}$ than for ${ }^{206} \mathrm{~Pb}$.

In Fig. 2.18, we compare the quadrupole deformation, $\beta_{2}$, of the ground states for sulfur isotopes predicted by the two models [67]. The deformation for even-even isotopes ${ }^{28-38} \mathrm{~S}$ is fairly similar in both SHF and RMF models, i.e., prolate $\left(\beta_{2}>0\right)$ ground states in ${ }^{28-32,38} \mathrm{~S}$, oblate minimum in ${ }^{34} \mathrm{~S}$, and spherical shape in the magic nucleus ${ }^{36} \mathrm{~S}$ with $\mathrm{N}=20$. However, there are significant differences in heavier isotopes, in particular, for ${ }^{40,42} \mathrm{~S}$. They are predicted by the RMF to have prolate ground states with $\beta_{2} \approx 0.25$; the oblate minima with $\beta_{2} \approx-0.16$ lie about $4 \mathrm{MeV}$ above the ground state. In the SHF calculations, however, prolate $\left(\beta_{2} \approx 0.25\right)$ and oblate $\left(\beta_{2} \approx-0.24\right)$ configurations are essentially degenerate.

A recent experiment at Michigan State University by Scheit et al. 68 has confirmed the predicted large deformation of ${ }^{40,42} \mathrm{~S}$, but could not distinguish the sign 


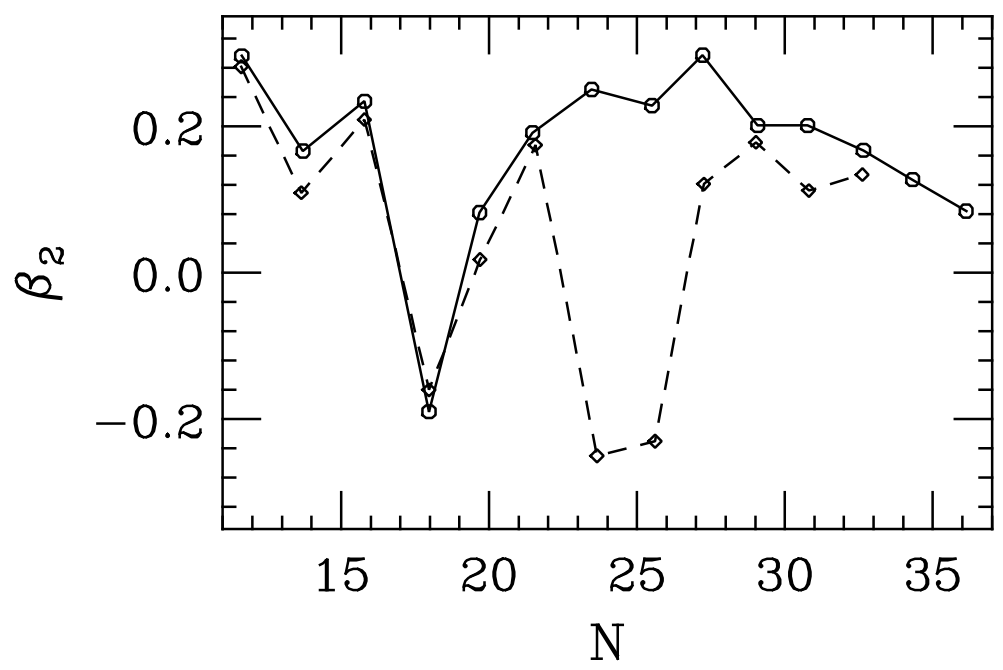

Figure 2.18: Quadrupole deformations of sulfur isotopes predicted by the SHF (dashed line connecting the squares) and RMF URCA (solid line connecting the circles) models. Results taken from Ref. [67].

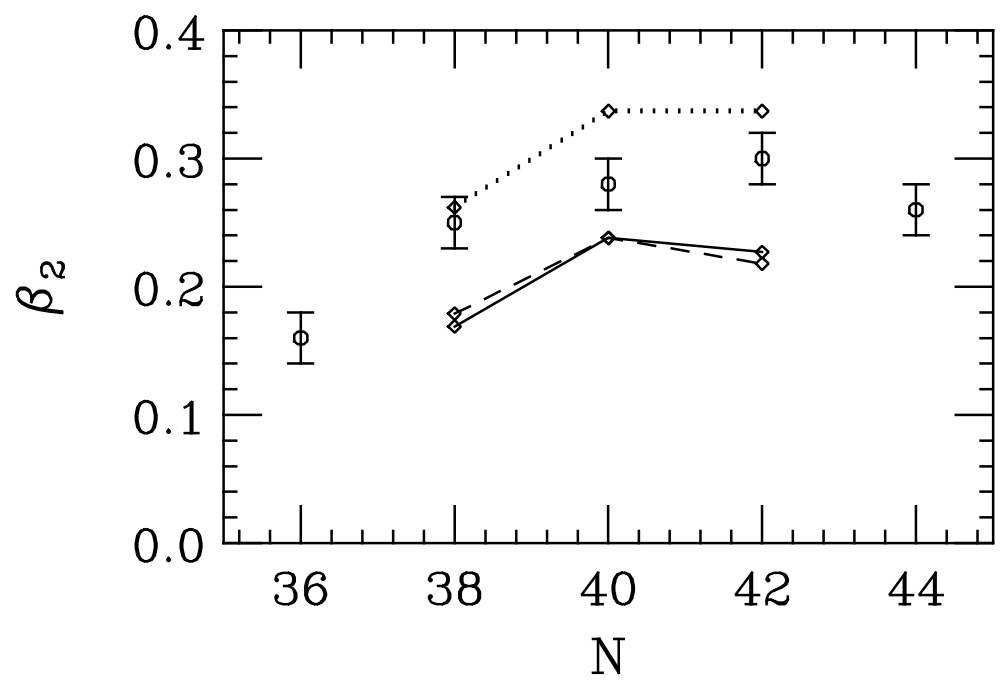

Figure 2.19: Comparison of the quadrupole deformations of sulfur isotopes. Points with error bars are the experimental data, while the dashed, solid, and dotted lines are the predictions of the SHF, RMF and shell model, respectively. Results taken from Ref. [68, 69]. 
of deformation. In this experiment the energies and $B\left(E 2 ; 0_{\text {g.s. }}^{+} \rightarrow 2_{1}^{+}\right)$values of the $2_{1}^{+}$states of ${ }^{38,40,42} \mathrm{~S}$ were measured by Coulomb excitations. In Fig. 2.19 the measured deformation $\left|\beta_{2}\right|$ is compared to the predictions of the SHF, RMF and shell model. Both, SHF and RMF calculations slightly underpredict the deformation, while the shell model of Brown slightly overpredicts the deformation. Clearly, additional information about the sign of deformation is needed to distinguish the prediction of these models about the nuclear EOS .

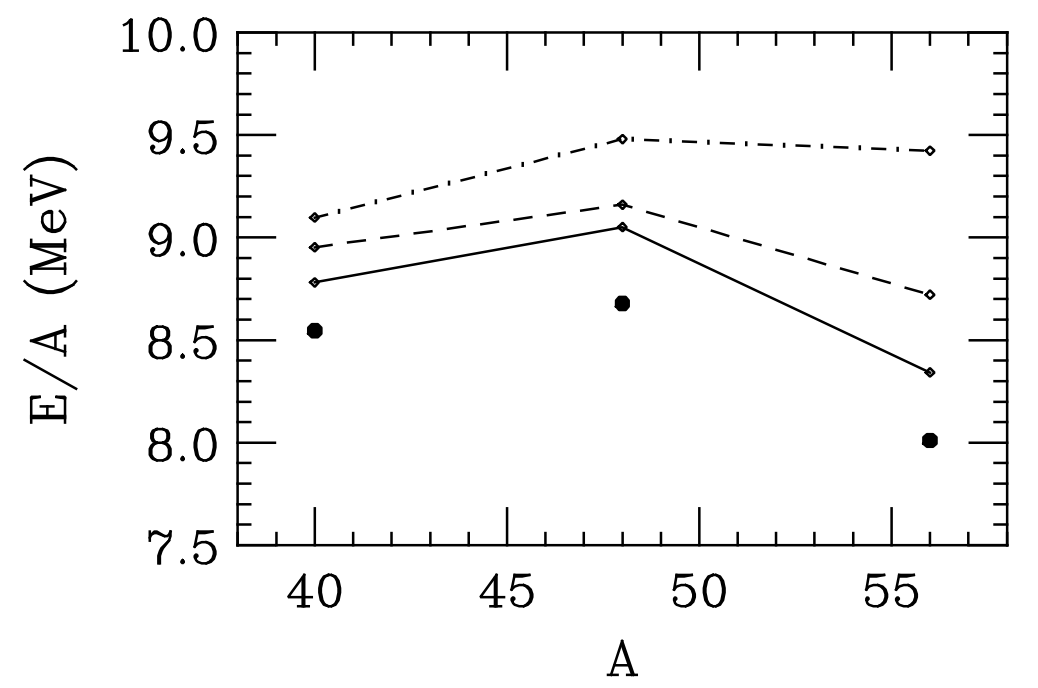

Figure 2.20: Binding energies of Ca isotopes. Solid points are the experimental data, while results from the Landau-Vlasov calculations with various values of $c(c=28$ : solid line, $c=20$ : dashed line, and $c=0$ : dot-dashed line) are shown as diamonds connected by lines. Results taken from Ref. [70].

Properties of neutron-rich nuclei have also been studied using the BoltzmannUehling-Uhlenbeck (BUU) or Landau-Vlasov (LV) models [70, 71]. These models, as we shall discuss in more detail in section 4.1, were originally developed for studying the reaction dynamics of heavy-ion collisions. They are based on the semi-classical approximation, and are thus suitable for studying the dependence of the gross properties of neutron rich nuclei on the isospin-dependent EOS.

To include the effects of isospin on nuclear dynamics, Farine et al. have added to the Zamick potential energy density, which has been widely used in standard BUU/LV models, an asymmetric term of the form [70]

$$
w_{\mathrm{sym}}=c \rho\left[\left(\rho_{n}-\rho_{p}\right) / \rho_{0}\right]^{2},
$$

where the coefficient $c=e_{\text {sym }}\left(\rho_{0}\right)-\frac{1}{3} E_{F}^{0}$ is the the symmetry energy at normal nuclear matter density due to nuclear interactions. They have studied the dependence of 


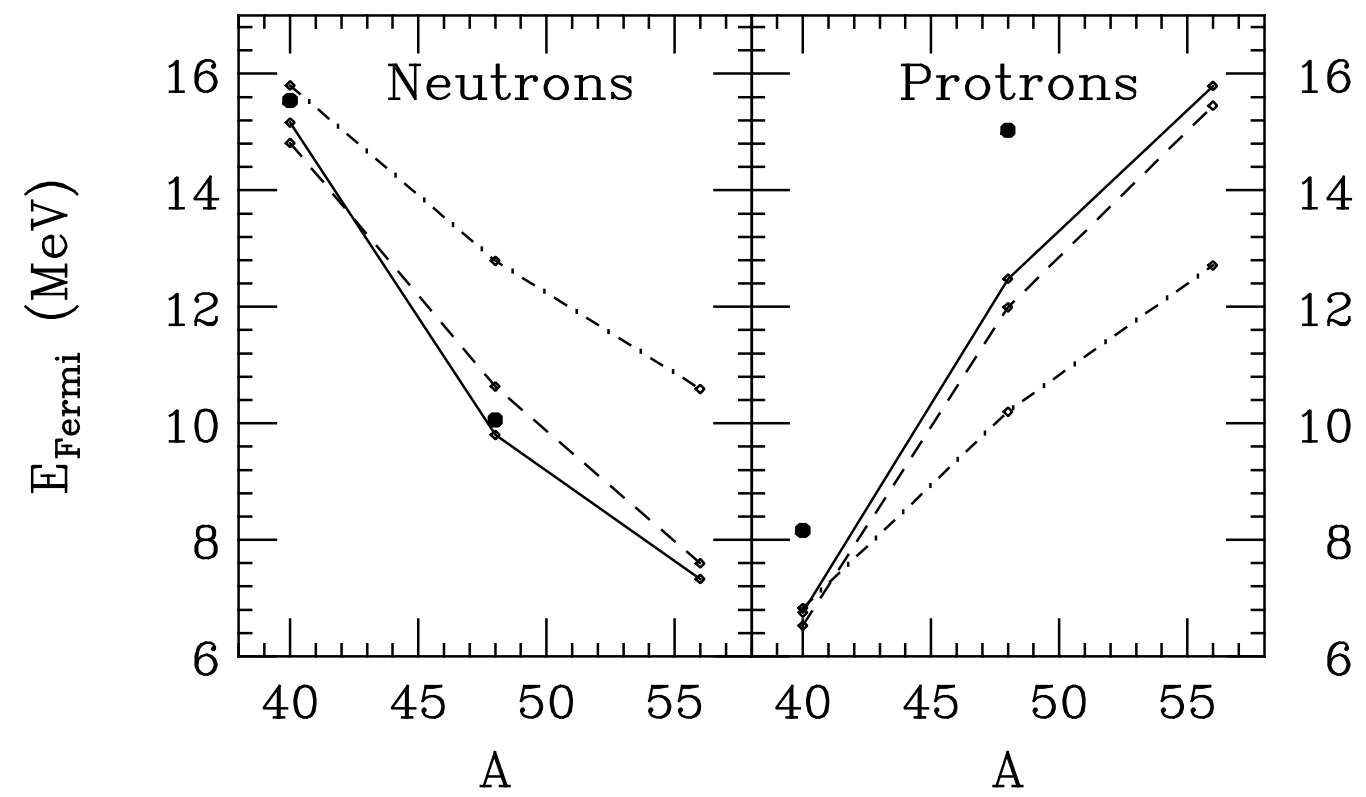

Figure 2.21: Same as Fig. 2.20 for the Fermi energies of protons and neutrons. Taken from Ref. [70].

ground state properties of neutron-rich Ca isotopes on the isospin-dependent EOS by varying the value of $c$. Fig. 2.20 shows a comparison of the calculated binding energies of three neutron-rich Ca isotopes with the experimental data shown by plus signs. The data for ${ }^{56} \mathrm{Ca}$ do not exist, its binding energy is obtained using the extended ThomasFermi with Strutinsky-integral model (ETFSI) of Pearson et al. [72]. As expected, nuclei become less bound with increasing $c$. Moreover, this effect is the strongest for the most neutron-rich nucleus ${ }^{56} \mathrm{Ca}$. However, the model tends to overbind nuclei for $c$ up to $28 \mathrm{MeV}$. The comparison of the Fermi energies of neutrons and protons for the three $\mathrm{Ca}$ isotopes are shown in Fig. 2.21. General features of the Fermi energies are seen to be correctly described by the LV model. The results from $c=$ 0 are clearly far from the data since the Coulomb energy of protons is then not properly balanced by the symmetry energy. As a result the density distribution of neutrons and protons obtained with $c=0$ is incorrect. Since the symmetry potential is repulsive for neutrons and attractive for protons, a stronger symmetry potential would deplete more neutrons from the center to the surface of a nucleus as noticed in Refs. 771, 73, 74, 75]. The demonstration by Sobotka using the BuU model and an isospin-dependent EOS based on the Skyrme II effective interaction is instructive [71]. The density distributions for neutrons and protons in ${ }^{197} \mathrm{Au}$ obtained from this study are shown in Fig. 2.22 for the case with (solid line) and without (dashed line) symmetry potential. Shown are (from top to bottom) the total nucleon density, the neutron density, and the proton density. In both cases, the total nucleon density distribution is the same. However, there are differences in the individual density distributions for protons and neutrons. Without the attractive symmetry potential 
for protons, they are pushed towards the surface by the Coulomb force, and there is almost no neutron skin. Including the symmetry potential, a neutron skin of about $1 / 3 \mathrm{fm}$ is seen, and this is consistent with the droplet model prediction [76].

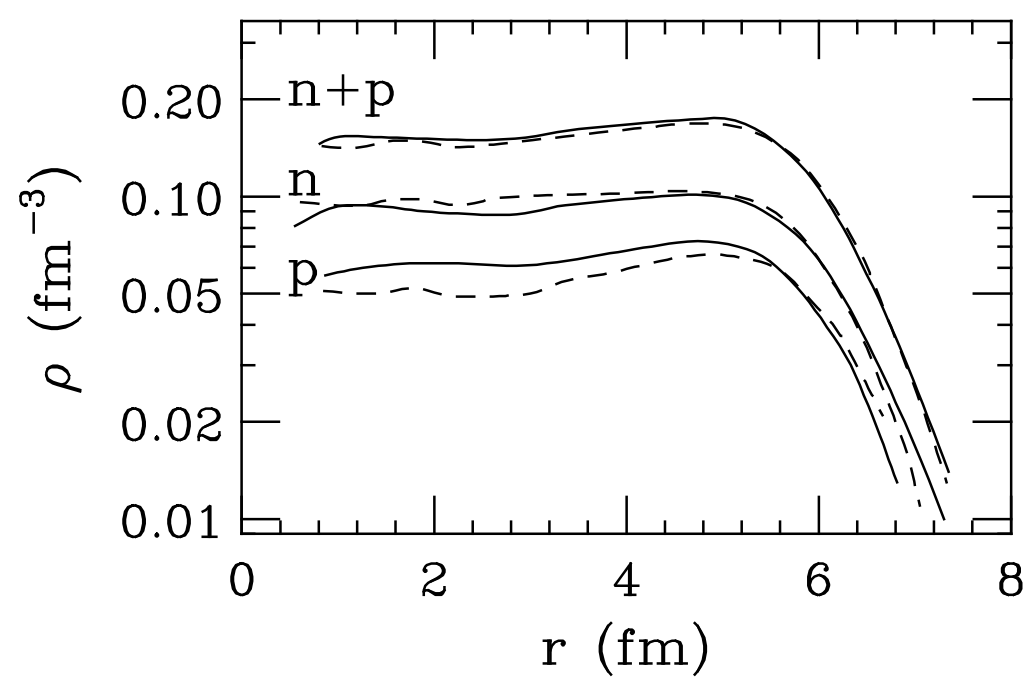

Figure 2.22: BUU model calculations of the neutron and proton densities for ${ }^{197} \mathrm{Au}$ with (solid lines) and without (dashed lines) symmetry energy. Shown are (from top to bottom) the total nucleon density, the neutron density, and the proton density. The calculations are from Ref. [71].

To summarize, properties of neutron-rich nuclei are sensitive to the the EOS of asymmetric nuclear matter at saturation density. However, to extract properties of isospin asymmetric nuclear matter at densities away from the saturation density collisions of neutron-rich nuclei will be more useful. Since semiclassical transport models can reproduce the gross properties of neutron-rich nuclei and their dependence on the isospin-dependent EOS, they thus provide a useful framework for studying collisions of neutron-rich nuclei and for extracting information about the isospindependence of the nuclear EOS.

\subsection{Chemical and mechanical instabilities in hot asymmetric nuclear matter}

Nuclear matter is not thermodynamically stable at all densities, $\rho$, temperatures, $T$, and neutron excesses, $\delta$. The necessary and sufficient conditions for the stability of an isospin-asymmetric nuclear matter can be expressed by the following inequalities 
[16, 77, 78]:

$$
\begin{aligned}
& \left(\frac{\partial E}{\partial T}\right)_{\rho, \delta}>0 \\
& \left(\frac{\partial P}{\partial \rho}\right)_{T, \delta} \geq 0, \\
& \left(\frac{\partial \mu_{n}}{\partial \delta}\right)_{P, T} \geq 0,
\end{aligned}
$$

where $E, P$ and $\mu_{n}$ are the energy per nucleon, pressure and neutron chemical potential, respectively. The first condition ensures the thermodynamic stability and is satisfied by any reasonable equation of state; the second condition prevents the mechanical instability against the growth of density fluctuations; and the last one protects the diffusive stability against the development of neutronization. The last two conditions might be violated in certain regions in the $(\rho, T, \delta)$ configuration space. Nuclear multifragmentations initiated by various mechanical instabilities, such as the volume instability, the surface instability of the Rayleigh kind, and the Coulomb instability, have been extensively studied during the last decade [79, 80, 81, 82]; for a recent review, see, e.g., [83]. With the recent advance in radioactive ion beams, a nuclear matter with a large isospin asymmetry can be created transiently in these reactions. It is thus of interest to study the chemical instability of isospin-asymmetric nuclear matter.

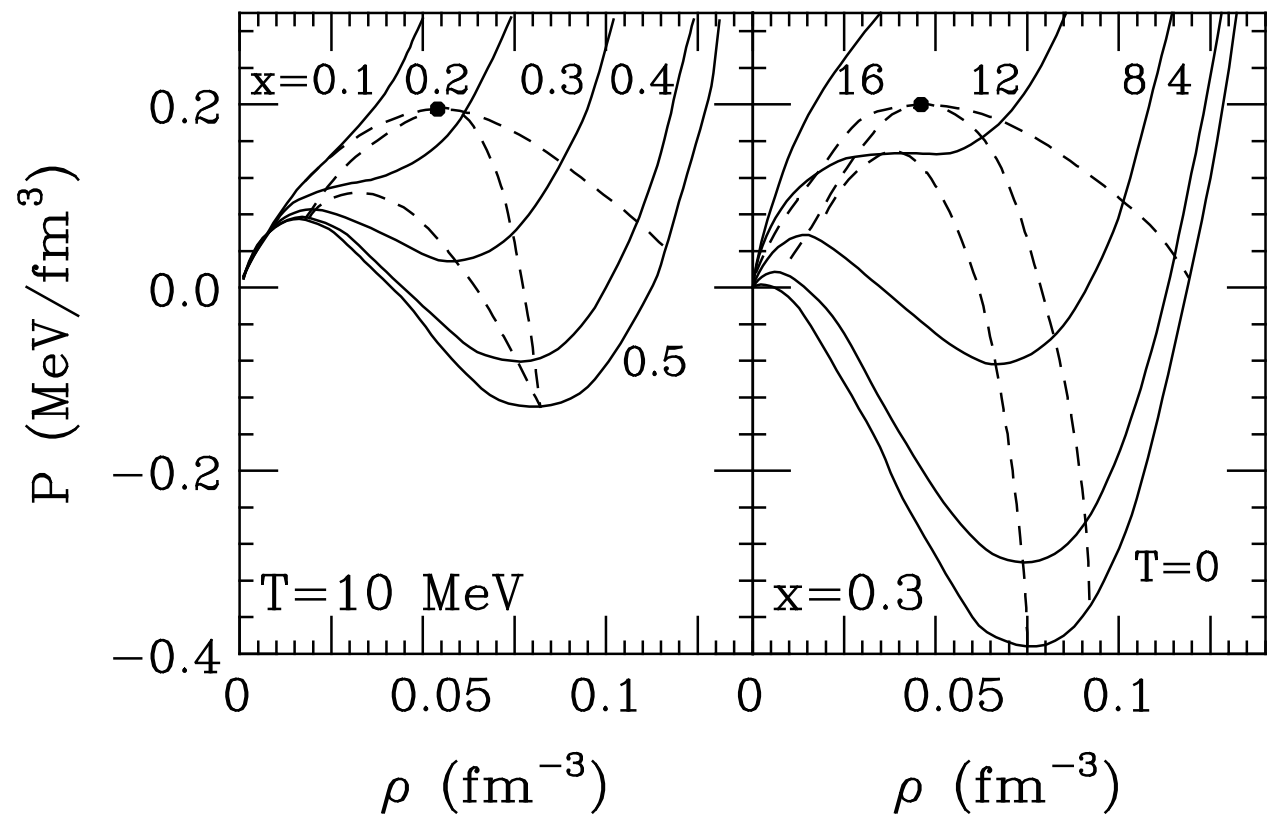

Figure 2.23: RMF predictions of pressure as a function of density at fixed temperature $T$ for various proton fraction $x$ (left window), and at fixed $x$ for various temperature $T$ (right window). Taken from Ref. [16]. 
To understand the respective roles of chemical and mechanical instabilities on the dynamics of nuclear matter, it is important to study the relative locations of these instabilities in the $(\rho, T, \delta)$ configuration space. Since the adiabatic spinodal is always enclosed by the isothermal spinodal (ITS), it is sufficient to compare the diffusion spinodal (DS) and ITS. Obviously, ; the boundaries of these instabilities depend on the equation of state. Using RMT theory Müller and Serot have found that the diffusive spinodal encloses more of the configuration space than the isothermal spinodal [16]. Fig. 2.23 shows typical results form their calculations at a constant temperature $T$ (left window) or a constant proton fraction $x$ (right window). At currently reachable region of $x \geq 0.3$ in neutron-rich nuclei induced reactions, the separation between the isothermal and diffusive spinodals in $P-\rho$ configuration space is appreciable.

As discussed in previous sections, the equation of state of asymmetric nuclear matter varies significantly among models. In particular, the RMF theory predicts a characteristic linear density dependence of the symmetry energy. It is therefore of interest to examine how the conclusions based on the RMF might change when other equations of state for asymmetric matter are considered. In the following we discuss the results for the chemical and mechanical instabilities in a thermal model using phenomenological equations of state. The advantage of a thermal model is that at modestly high temperatures, most quantities relevant to the discussion of chemical and mechanical instabilities can be evaluated analytically.

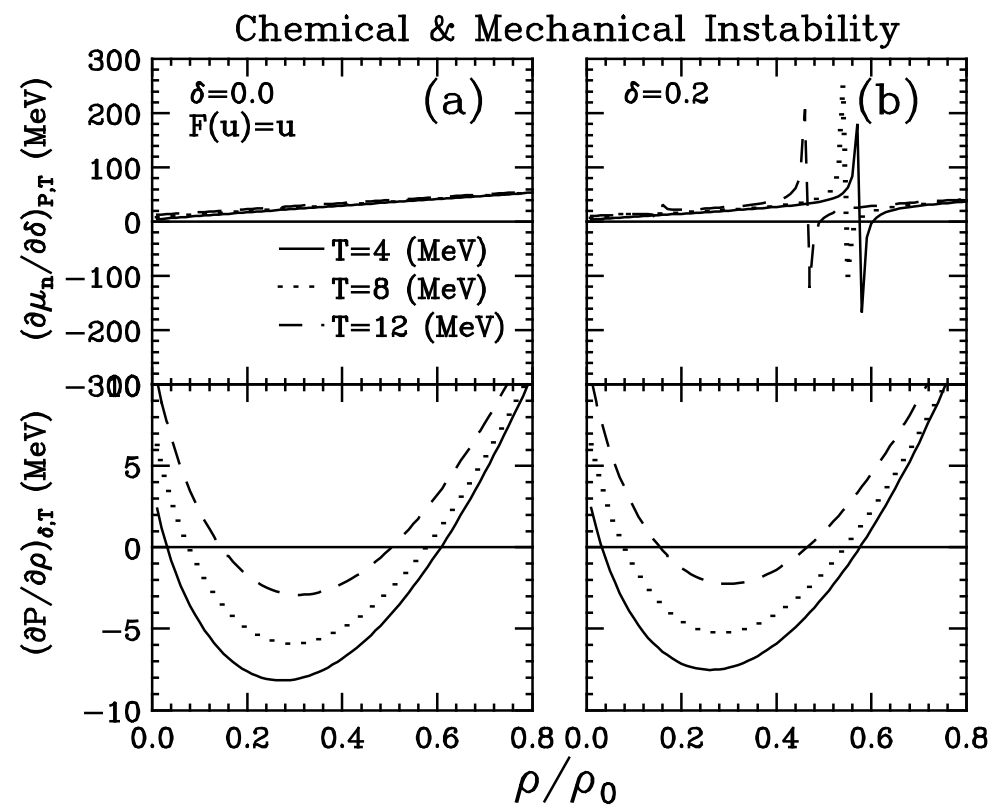

Figure 2.24: Chemical (upper window) and mechanical (lower window) stability conditions as functions of density at fixed temperatures $T=4,8$ and $12 \mathrm{MeV}$ for $\delta=0.0$ (left panel) and $\delta=0.2$ (right panel). Taken from Ref. 87]. 


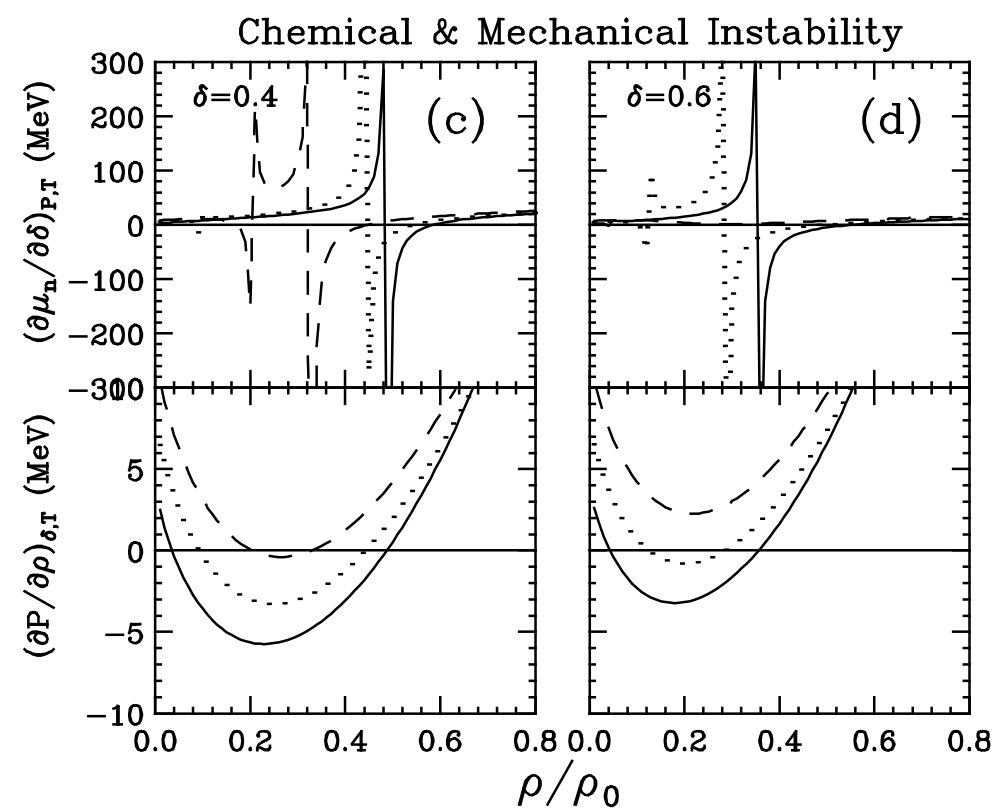

Figure 2.25: Same as Fig. 2.24 for $\delta=0.4$ (left panel) and $\delta=0.6$ (right panel). Taken from Ref. [87].

The density $\rho_{q}$ of neutrons $(q=n)$ or protons $(q=p)$ is

$$
\rho_{q}=\frac{1}{\pi^{2}} \int_{0}^{\infty} k^{2} f_{q}(k) d k,
$$

where

$$
f_{q}(k)=\left[\exp \left(e_{q}-\mu_{q}\right) / T+1\right]^{-1}
$$

is the nucleon Fermi distribution function, and $e_{q}$ is the single particle kinetic energy. At modestly high temperatures $(T \geq 4 M e V)$, this equation can be inverted analytically to obtain the chemical potential [84, 85, 86]

$$
\mu_{q}=V_{q}+T\left[\ln \left(\frac{\lambda_{T}^{3} \rho_{q}}{2}\right)+\sum_{n=1}^{\infty} \frac{n+1}{n} b_{n}\left(\frac{\lambda_{T}^{3} \rho_{q}}{2}\right)^{n}\right],
$$

where

$$
\lambda_{T}=\left(\frac{2 \pi \hbar^{2}}{m T}\right)^{1 / 2}
$$

is the thermal wavelength of the nucleon, and $b_{n}^{\prime} s$ are inversion coefficients. In Eq. (2.30) $V_{q}$ is the single particle potential energy and can be parameterized as

$$
V_{q}(\rho, \delta)=a\left(\rho / \rho_{0}\right)+b\left(\rho / \rho_{0}\right)^{\sigma}+V_{\mathrm{asy}}^{q}(\rho, \delta) .
$$


The parameters $a, b$ and $\sigma$ are determined by the saturation properties and the compressibility $K$ of symmetric nuclear matter, i.e.,

$$
\begin{aligned}
a & =-29.81-46.90 \frac{K+44.73}{K-166.32}(\mathrm{MeV}), \\
b & =23.45 \frac{K+255.78}{K-166.32}(\mathrm{MeV}) \\
\sigma & =\frac{K+44.73}{211.05} .
\end{aligned}
$$

The isospin-independent term should also contain a momentum-dependent part which is important for certain dynamical observables in heavy ion collisions, such as the collective flow (e.g., 88, 89, 90), but is not essential for observables such as the neutron to proton ratio of preequilibrium nucleons [91]. Effects of the momentumdependent interactions on thermal properties have been recently studied in Refs. [92, 93, and rather small effects on the global properties of nuclei are found. Here we neglect the momentum-dependent interaction and shall concentrate on investigating the effects of the isospin-asymmetric potential $V_{\text {asy }}^{q}$. This potential is given by

$$
V_{\mathrm{asy}}^{q}(\rho, \delta)=\partial w_{a}(\rho, \delta) / \partial \rho_{q},
$$

where $w_{a}(\rho, \delta)$ is the contribution of nuclear interactions to the symmetry energy density, i.e.,

$$
w_{a}(\rho, \delta)=e_{a} \cdot \rho F(u) \delta^{2}
$$

and

$$
e_{a} \equiv e_{\mathrm{sym}}\left(\rho_{N M}\right)-\left(2^{2 / 3}-1\right) \frac{3}{5} E_{F}^{0} .
$$

Using the three forms of $F(u)$ discussed in section 2.3, i.e., $F(u)=u^{2}, u$ and $u^{1 / 2}$, the corresponding symmetry potentials are, respectively,

$$
\begin{aligned}
& V_{\text {asy }}^{n(p)}= \pm 2 e_{a} u^{2} \delta+e_{a} u^{2} \delta^{2}, \\
& V_{\text {asy }}^{n(p)}= \pm 2 e_{a} u \delta,
\end{aligned}
$$

and

$$
V_{\mathrm{asy}}^{n(p)}= \pm 2 e_{a} u^{1 / 2} \delta-\frac{1}{2} e_{a} u^{1 / 2} \delta^{2} .
$$

With the chemical potentials determined by Eq. (2.30), it is then possible to obtain the total pressure of the nuclear matter by using the Gibbs-Duhem relation

$$
\frac{\partial P}{\partial \rho}=\frac{\rho}{2}\left[(1+\delta) \frac{\partial \mu_{n}}{\partial \rho}+(1-\delta) \frac{\partial \mu_{p}}{\partial \rho}\right] .
$$

The pressure can be separated into three parts, i.e.,

$$
P=P_{\text {kin }}+P_{0}+P_{\text {asy }},
$$


where $P_{\text {kin }}$ is the kinetic contribution

$$
P_{\text {kin }}=T \rho\left[1+\frac{1}{2} \sum_{n=1}^{\infty} b_{n}\left(\frac{\lambda_{T}^{3} \rho}{4}\right)^{n}\left((1+\delta)^{n+1}+(1-\delta)^{1+n}\right)\right],
$$

and $P_{0}$ is the contribution from the isospin-independent nuclear interaction

$$
P_{0}=\frac{1}{2} a \rho_{0}\left(\frac{\rho}{\rho_{0}}\right)^{2}+\frac{b \sigma}{1+\sigma} \rho_{0}\left(\frac{\rho}{\rho_{0}}\right)^{\sigma+1} .
$$

$P_{\text {asy }}$ is the contribution from the isospin-dependent part of the nuclear interaction, and can be written as

$$
\begin{aligned}
& P_{\text {asy }}=2 e_{a} \rho_{0}\left(\frac{\rho}{\rho_{0}}\right)^{3} \delta^{2}, \\
& P_{\text {asy }}=e_{a} \rho_{0}\left(\frac{\rho}{\rho_{0}}\right)^{2} \delta^{2},
\end{aligned}
$$

and

$$
P_{\text {asy }}=\frac{1}{2} e_{a} \rho_{0}\left(\frac{\rho}{\rho_{0}}\right)^{3 / 2} \delta^{2}
$$

for $F(u)=u^{2}, u$ and $u^{1 / 2}$, respectively.

From the chemical potential and pressure, the stability conditions can be determined. The determination of the mechanical stability condition is straightforward. However, to calculate $\partial \mu_{n} / \partial \delta$ at constant pressure $P$ and neutron excess $\delta$, the following transformation is useful:

$$
\begin{aligned}
\left(\frac{\partial \mu_{n}}{\partial \delta}\right)_{T, P} & =\left(\frac{\partial \mu_{n}}{\partial \delta}\right)_{T, \rho}-\left(\frac{\partial \mu_{n}}{\partial P}\right)_{T, \delta} \cdot\left(\frac{\partial P}{\partial \delta}\right)_{T, \rho} \\
& =\left(\frac{\partial \mu_{n}}{\partial \delta}\right)_{T, \rho}-\left(\frac{\partial \mu_{n}}{\partial \rho}\right)_{T, \delta} \cdot\left(\frac{\partial P}{\partial \rho}\right)_{T, \delta}^{-1} \cdot\left(\frac{\partial P}{\partial \delta}\right)_{T, \rho}
\end{aligned}
$$

Knowing $\left(\partial \mu_{n} / \partial \delta\right)_{T, P}$ and $(\partial P / \partial \rho)_{T, \delta}$ as functions of $\rho, T$, and $\delta$, regions of chemical and/or mechanical instability can then be identified in the configuration space. First, we show in Fig. 2.24 and Fig. 2.25 these two quantities as functions of $\rho$ at various $T$ and $\delta$. In this calculation $K=200 \mathrm{MeV}$ and $F(u)=u$ are used. For comparison results for symmetric nuclear matter are shown in the left window of Fig. 2.24. In this case there is no chemical instability and the quantity $\left(\partial \mu_{n} / \partial \delta\right)_{T, P}$ increases with both density and temperature. To understand this, we note that

$$
\begin{aligned}
\left(\frac{\partial P}{\partial \delta}\right)_{T, \rho} & =\frac{2}{\delta} P_{\mathrm{asy}}+\frac{T \rho}{2} \sum_{n=1}^{\infty}(n+1) b_{n}\left(\frac{\lambda_{T}^{3} \rho}{4}\right)^{n}\left[(1+\delta)^{n}-(1-\delta)^{n}\right] \\
\left(\frac{\partial \mu_{n}}{\partial \delta}\right)_{T, \rho} & =\left(\frac{\partial V_{a s y}^{n}}{\partial \delta}\right)_{T, \rho} \\
& +T\left[\frac{1}{1+\delta}+\sum_{n=1}^{\infty}(n+1) b_{n}\left(\frac{\lambda_{T}^{3} \rho}{4}\right)^{n}(1+\delta)^{n+1}\right],
\end{aligned}
$$


and

$$
\lim _{\delta \rightarrow 0}\left(\frac{\partial P}{\partial \delta}\right)_{T, \rho}=0
$$

Then, it is easy to show that

$$
\begin{aligned}
& \lim _{\delta \rightarrow 0}\left(\frac{\partial \mu_{n}}{\partial \delta}\right)_{T, P}=\lim _{\delta \rightarrow 0}\left(\frac{\partial \mu_{n}}{\partial \delta}\right)_{T, \rho}, \\
& =2 e_{a} F(u)+T\left[1+\sum_{n=1}^{\infty}(n+1) b_{n}\left(\frac{\lambda_{T}^{3} \rho}{4}\right)^{n}\right],
\end{aligned}
$$

which is positive as one expects for symmetric nuclear matter and increases with both density and temperature.

The mechanical instability is known to occur at intermediate densities, i.e., in the mixed phase, between the gas and liquid phases at subcritical temperatures. This is clearly demonstrated by plotting $(\partial P / \partial \rho)_{T, \delta}$ as a function of $\rho$. It is interesting to examine this quantity in the limit of low densities. From the Gibbs-Duhem relation of Eq. (2.42) and the expression for $\mu_{q}$ of Eq. (2.30), one has

$$
\lim _{\rho \rightarrow 0}\left(\frac{\partial P}{\partial \rho}\right)_{T, \delta}=\lim _{\rho \rightarrow 0}\left(T+a \frac{\rho}{\rho_{0}}\right)=T,
$$

as expected for an ideal gas.

We note from Eq. (2.49) that the quantity $\left(\partial \mu_{n} / \partial \delta\right)_{T, P}$ is singular along the boundary for mechanical instability, where $(\partial P / \partial \rho)_{T, \delta}=0$. This singularity is indicated by the spikes in Fig. 2.24 and Fig. 2.25. However, their heights at the boundary of the gas and mixed phases is very small because the last derivative in Eq. (2.49) is close to zero, i.e.,

$$
\lim _{\rho \rightarrow 0}\left(\frac{\partial P}{\partial \delta}\right)_{T, \rho}=\frac{2}{\delta} P_{\text {asy }}=0 .
$$

Furthermore, in the ideal gas limit the nuclear matter is also chemically stable, i.e.,

$$
\lim _{\rho \rightarrow 0}\left(\frac{\partial \mu_{n}}{\partial \delta}\right)_{P, T}=\frac{T}{1+\delta}>0 .
$$

As the system becomes neutron-rich, the chemical instability starts to develop, so the boundary for mechanical instability shrinks while that for chemical instability expands as $\delta$ increases. For example, in contrast to the variation of the chemical instability the mechanical instability at a temperature of $\mathrm{T}=10 \mathrm{MeV}$ gradually disappears as $\delta$ increases from 0 to 0.6 . Furthermore, in the mechanically unstable region the system is chemically stable, but at higher densities chemical instability can appear in the mechanically stable region. Therefore, in neutron-rich nuclear matter the chemical instability is a more relevant quantity in determining its properties. As shown in Fig. 2.26, where the pressure along the chemical, (DS), and mechanical, (ITS), spinodals are plotted as functions of density, both the isothermal pressure and 


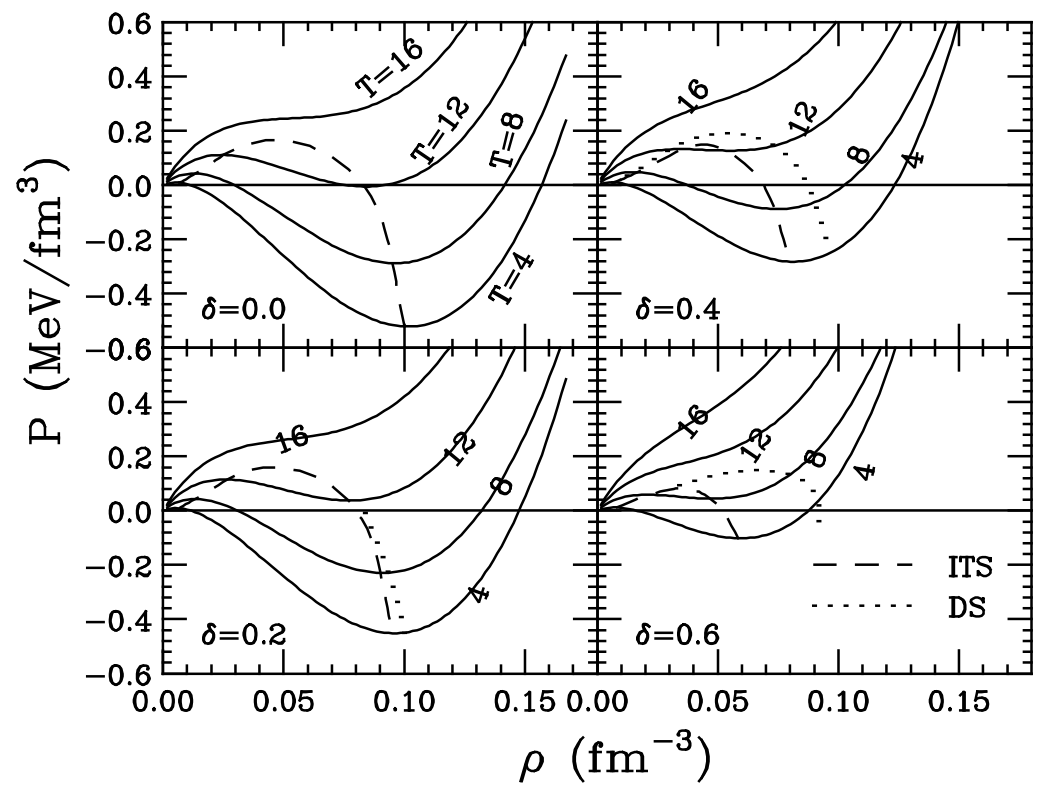

Figure 2.26: Pressure as a function of density at fixed temperatures $T=4,8,12$ and $16 \mathrm{MeV}$ for $\delta=0.0,0.2,0.4$ and 0.6. The isothermal and diffusive spinodals are shown by the dashed and dotted lines, respectively. Taken from Ref. [87.

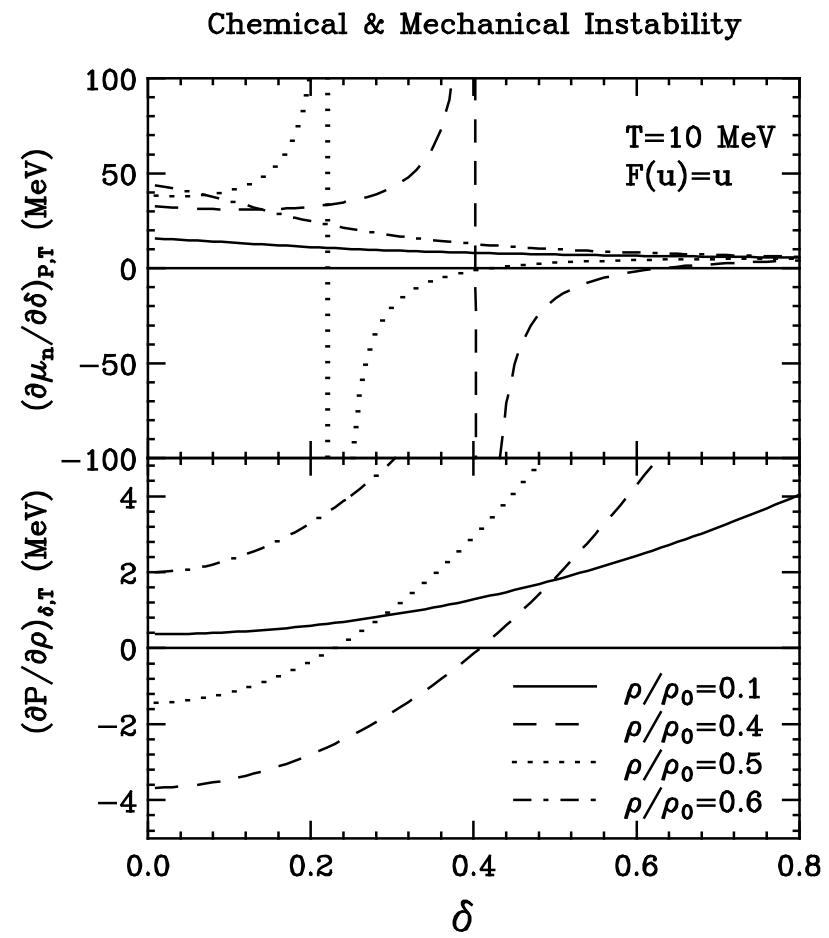

Figure 2.27: Chemical (upper window) and mechanical (lower window) instability conditions as functions of $\delta$ at a fixed temperature $T=10 \mathrm{MeV}$ and various densities. Taken from Ref. 87]. 


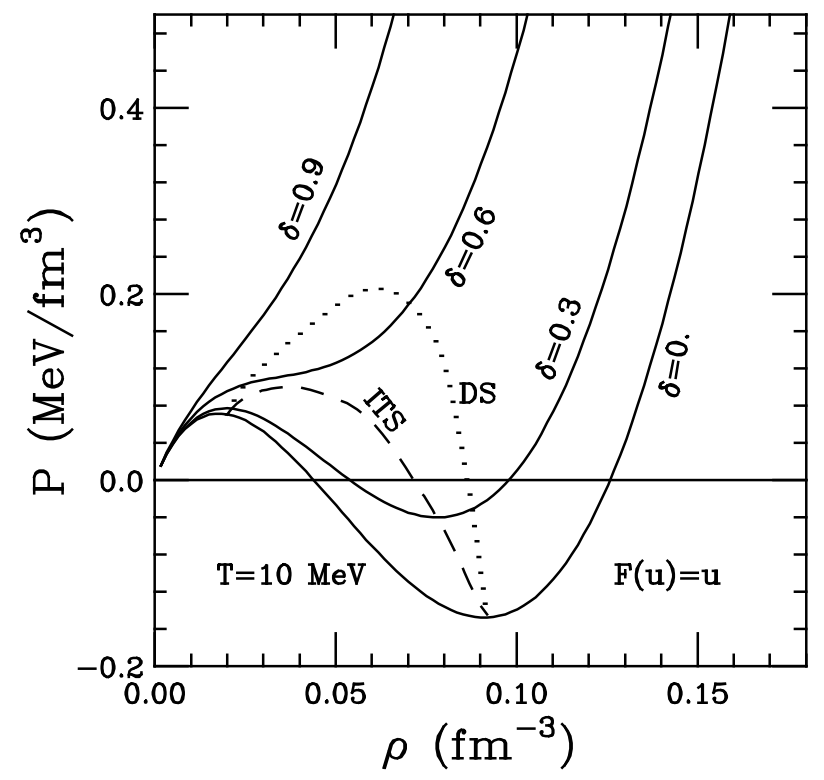

Figure 2.28: Pressure as a function of density at a fixed temperature $T=10 \mathrm{MeV}$ at various $\delta$. The isothermal and diffusive spinodals are shown by the dashed and dotted lines, respectively. Taken from Ref. [87.

the separation between the two spinodals increase with increasing $\delta$, so not only the chemical but also the mechanical instability is strongly isospin-dependent.

The isospin-dependence of mechanical and chemical instabilities at a fixed temperature are studied in more detail in Fig. 2.27 and Fig. 2.28. It is seen that nuclear matter is both mechanically and chemically stable at low and high densities. At intermediate densities (e.g., $\rho / \rho_{0}=0.4,0.5$ ) the system is mechanically unstable for small $\delta$ (e.g., $\delta \leq 0.2$ and $\delta \leq 0.4$ for $\rho / \rho_{0}=0.4$ and 0.5 , respectively.), and chemically unstable for intermediate $\delta$ (e.g. $0.2 \leq \delta \leq 0.4$ for $\rho / \rho_{0}=0.4$ and $0.4 \leq \delta \leq 0.6$ for $\rho / \rho_{0}=0.5$, respectively.) The corresponding boundaries in the pressure-density space are shown in Fig. 2.28. Again, the diffusive spinodal is more extended than the isothermal spinodal. The dependence of pressure on isospin is also shown in this figure. Its strong dependence on $\delta$ is due to the significant $P_{\text {asy }}$ contribution to the total pressure.

The above analysis thus indicates a strong isospin-dependence of both chemical and mechanical instabilities. It is therefore of interest to know how this dependence might be affected by the isospin-dependent and -independent parts of the nuclear equation of state. This is illustrated in Fig. 2.29 and Fig. 2.30, where the pressure is shown as a function of density (left panels) and $\delta$ (right panels) along the diffusive spinodals (upper windows) and isothermal spinodals (lower windows) at a constant temperature of $T=10 \mathrm{MeV}$ by using the three forms of $F(u)$ and a compressibility of 380 and $200 \mathrm{MeV}$, respectively. Several interesting observations can be made from these two figures. First, the diffusive spinodals are always more extended in 

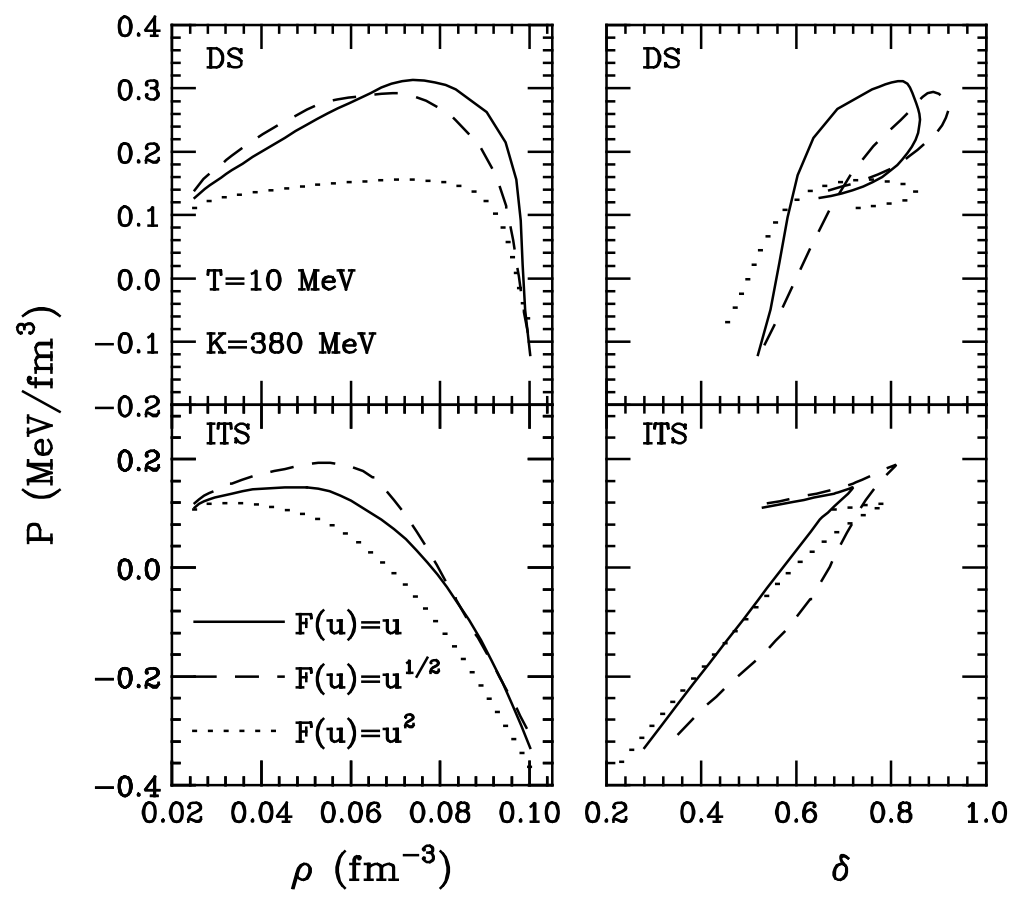

Figure 2.29: Pressure as a function of density (left panel) and isospin asymmetry (right panel) at a fixed temperature $T=10 \mathrm{MeV}$ along the boundary of diffusive spinodals (upper windows) and isothermal spinodals (lower windows) by using the three forms of $F(u)$ and a compressibility of $K=380 \mathrm{MeV}$. Taken from Ref. [87]. 

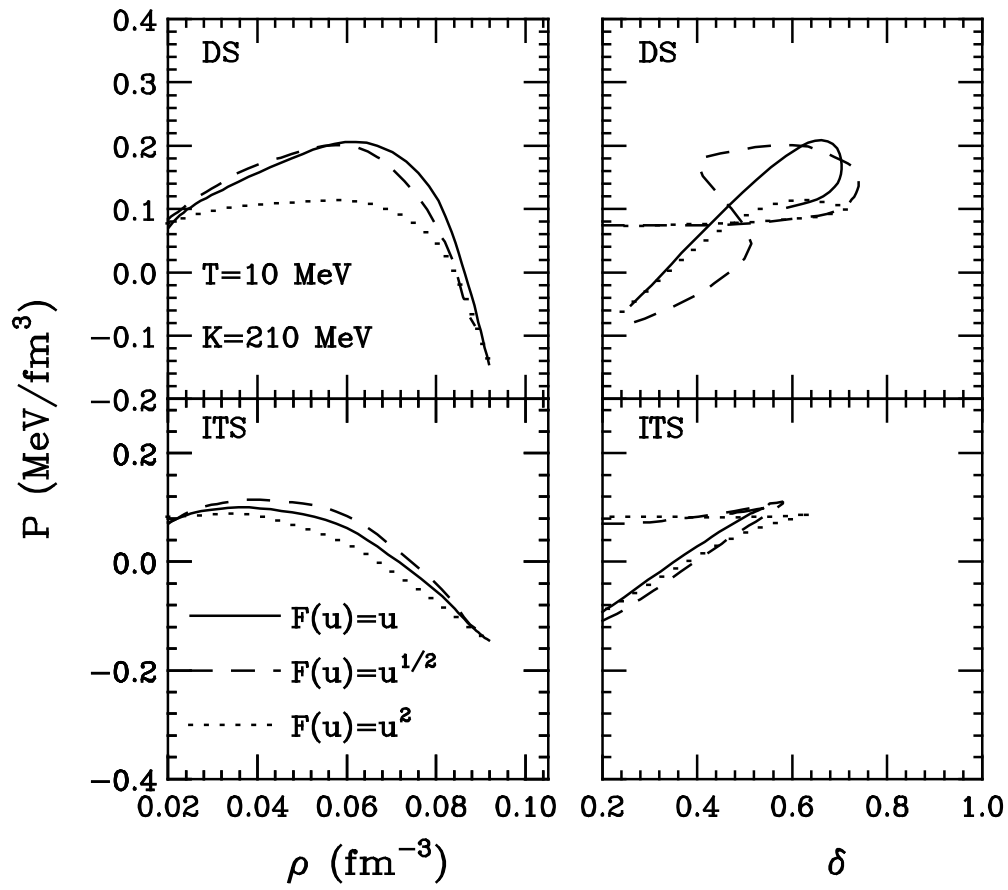

Figure 2.30: Same as Fig. 2.29 for a compressibility of $K=200 \mathrm{MeV}$. Taken from Ref. [87]. 
the configuration space than the isothermal spinodals. Second, both DS and ITS depend on the form of $F(u)$; and this dependence is stronger for the stiff equation of state with $K=380 \mathrm{MeV}$. Third, from the right panels of these two figures one sees that it is both chemically and mechanically favorable for nuclear matter to be less asymmetric (smaller $\delta$ ) in the liquid phase than in the gas phase. This result can also be obtained from energy considerations. Since the equation of state for asymmetric nuclear matter contains a $e_{\mathrm{sym}}(\rho) \delta^{2}$ term, it is therefore energetically favorable for asymmetric matter to separate into a liquid phase that is less asymmetric and a gas phase that is more asymmetric, rather than into two phases with equal asymmetry. Finally, there is a maximum asymmetry for both DS and ITS. As the density decreases from the liquid phase the asymmetry increases along both DS and ITS to a maximum value. The asymmetry then decreases towards the gas phase. As shown in these figures, the maximum asymmetry is also sensitive to both the isospin-dependent and -independent parts of the nuclear equation of state.

The results discussed in this section are more than just of academic interest. Significant differences have been observed in the multifragmentation of isospin-symmetric and isospin-asymmetric nuclear matter formed in heavy ion collisions at intermediate energies 94, 95, 96, 97. BUU calculations suggest that the abundance of neutron rich isotopes observed in experiments is primarily the result of the coalescence of the clusters with neutrons from the gas phase 97. That these neutron exist in abundance is a direct consequence of the large isospin-asymmetry in the gas phase. It is interesting, by the way, that the percolation model has difficulties in explaining the fragmentation data for neutron-rich isotopes [98], despite its success in matching a large number of other oberservables in nuclear fragmentation.

One popular explanation for nuclear multifragmentation is that the hot nuclear matter formed in the reaction expands adiabatically into the mechanical instability region where it fragments into clusters and nucleons due to the growth of density fluctuations 99. In describing this process great efforts have been devoted to incorporating effects of density fluctuations into dynamical models during the last decade 100, 101, 102, 103, 104. To describe multifragmentation in asymmetric nuclear collisions using these models, it will be necessary to extend them to include the isospin degree of freedom and its fluctuations. This will then allow one to compare the strengths of both density and isospin fluctuations and to study the relative time scales of chemical and mechanical instabilities in asymmetric nuclear matter. Indeed some interesting new features about the collective motions new the $\mathrm{n} / \mathrm{p}$ drip lines and the onset of chemical and mechanical instabilities have been found in recent studies using RPA 105 and Landau dispersion relation approaches 106].

\subsection{New features of liquid-gas phase transition in asymmetric nuclear matter}

The liquid-gas phase transition in asymmetric nuclear matter is not only more complex than in symmetric matter but also has new distinct features. This is because the 
nature of phase transitions in a matter is strongly influenced by its dimensionality. The inclusion of the isospin degree of freedom in studying the phase transitions in nuclear matter has thus attracted much attention recently 116, 56, 77, 78, 107, 108, 109, 110. These studies have shown that there are indeed new features associated with the liquid-gas phase transition in asymmetric nuclear matter.

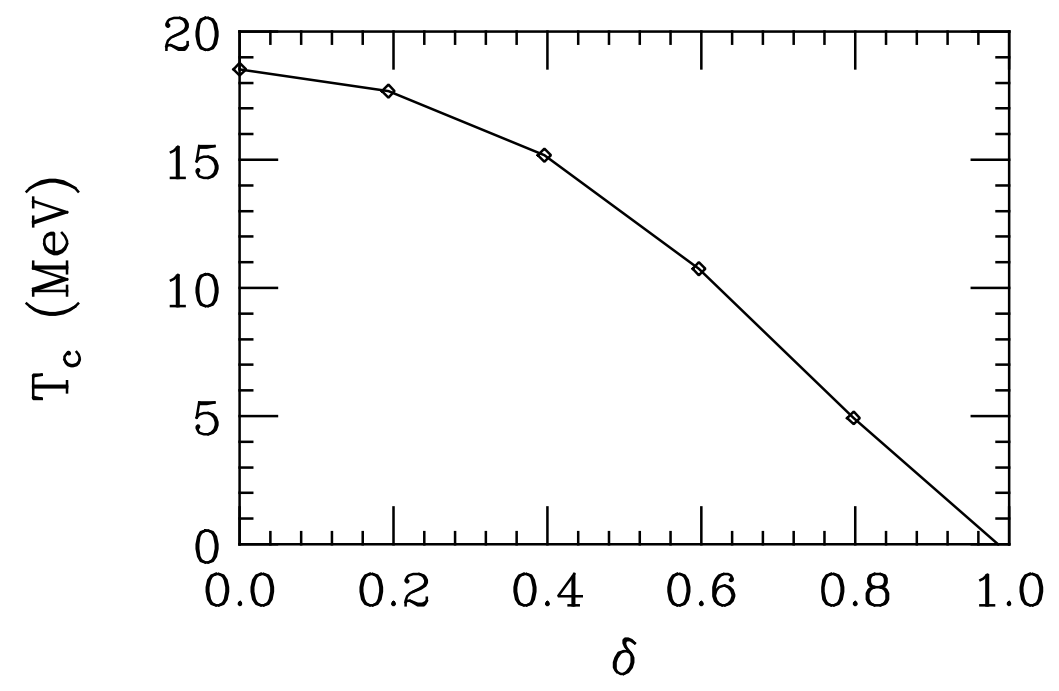

Figure 2.31: Critical temperature as a function of the asymmetry parameter $\delta$ from the isospin lattice gas model. Calculations taken from Ref. 110.

First of all, the critical temperature for the liquid-gas phase transition has been predicted to decrease with increasing neutron excess. This can be understood qualitatively in terms of the increasing contribution from the asymmetric pressure $P_{\text {asy }}$. Fig. 2.31 shows the critical temperature as a function of neutron-excess $\delta$ predicted by the isospin lattice gas model of Kuo et al. [110]. This prediction is in qualitative agreement with that based on the Skyrme interaction [77] and RMF theory [16]. Using the latter theory, Müller and Serot have studied the sensitivity of the critical point to the variation of the symmetry energy $e_{\mathrm{sym}}\left(\rho_{0}\right)$ and the bulk compressibility $K$. In Fig. 2.32 the binodal sections predicted by the RMF theory at $T=10 \mathrm{MeV}$ for $e_{\text {sym }}\left(\rho_{0}\right)=30,35$ and $40 \mathrm{MeV}$ are shown by the solid, dotted and dashed curves, respectively. As one would expect from our discussions in the previous section, the minimum proton fraction at which the phase separation occurs increases only by $10 \%$, and the critical temperature decreases by less than $1 \%$ as the symmetry energy increases from $e_{\text {sym }}\left(\rho_{0}\right)=30$ to $45 \mathrm{MeV}$. On the other hand, the critical pressure increases by about $40 \%$. However, the shape of binodal surfaces are rather similar for different values of symmetry energy. As we have stressed earlier, in the RMF theory the symmetry energy has a characteristic linear density dependence. Other models with different density dependence in the symmetry energy naturally may predict critical 


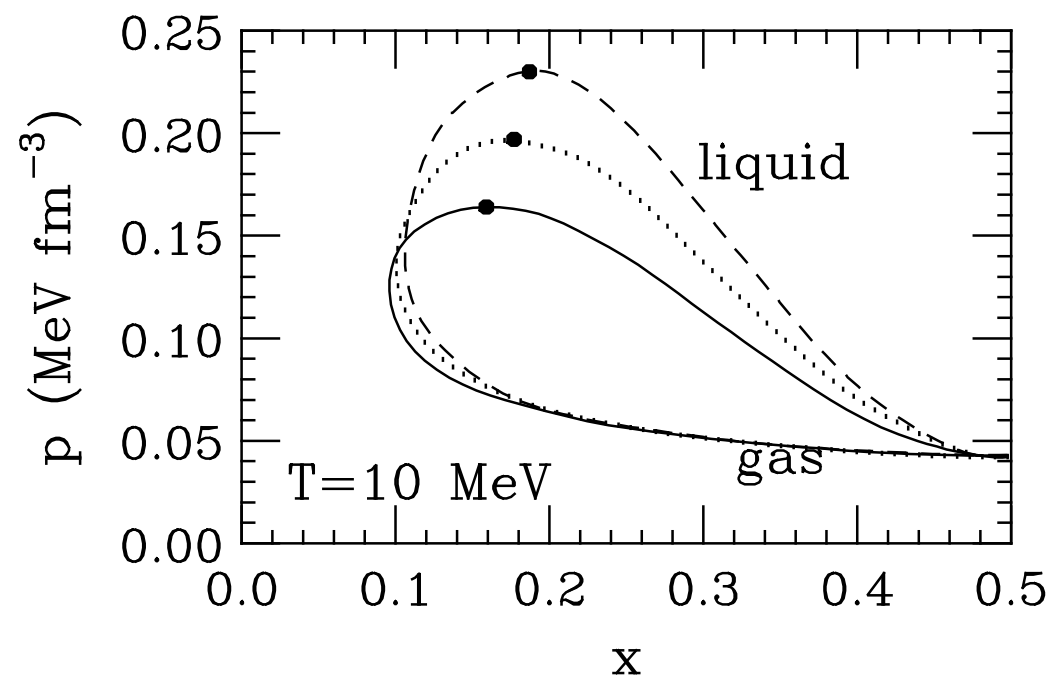

Figure 2.32: Binodal sections at $T=10 \mathrm{MeV}$ and $e_{\text {sym }}\left(\rho_{0}\right)=30,35$ and $40 \mathrm{MeV}$ shown by the solid, dotted and dashed curves, respectively. Taken from Ref. [16].

points at different temperature, pressure and proton fraction. It is well-known that the critical point of liquid-gas phase transition is sensitive to the bulk compressibility $K$. Müller and Serot have found that the critical temperature changes by about $13 \%$ when $K$ varies from 200 to $300 \mathrm{MeV}$ using the same symmetry energy $e_{\text {sym }}\left(\rho_{0}\right)=35$ $\mathrm{MeV}$. Although this variation is larger than that due to the variation of the symmetry energy, it is unlikely to have a significant effect on the liquid-gas phase transition.

An important question about the liquid-gas phase transition in asymmetric nuclear matter is its order. Glendenning [109] as well as Müller and Serot [16] have recently stressed that the isospin degree of freedom plays an important role in phase transitions. In the liquid-gas phase transition, not only the total baryon density but also the total charge density is conserved. For an asymmetric matter, the latter constraint leads to a smoother variation of the thermodynamical quantities, such as the temperature, specific entropy, and heat capacity, during the phase transition. The order of phase transition in asymmetric matter is then expected to be higher than that in symmetric nuclear matter. In Fig. 2.33, the variation of temperature and specific entropy for proton fractions of $0.5,0.45,0.4$ and 0.3 are shown. In the symmetric case, the specific entropy increases by 3 units in a temperature interval of $1.5 \mathrm{MeV}$, which increases to about $5 \mathrm{MeV}$ as the proton fraction decreases to 0.35 . Thus, both the specific entropy and temperature change continuously during the phase transition, so the heat transferred to the system is used not only to convert the liquid to vapor but also to heat the matter. The first-order liquid-gas phase transition in symmetric nuclear matter therefore changes to a second-order one in asymmetric nuclear matter.

Another important question about the liquid-gas phase transition in asymmetric 


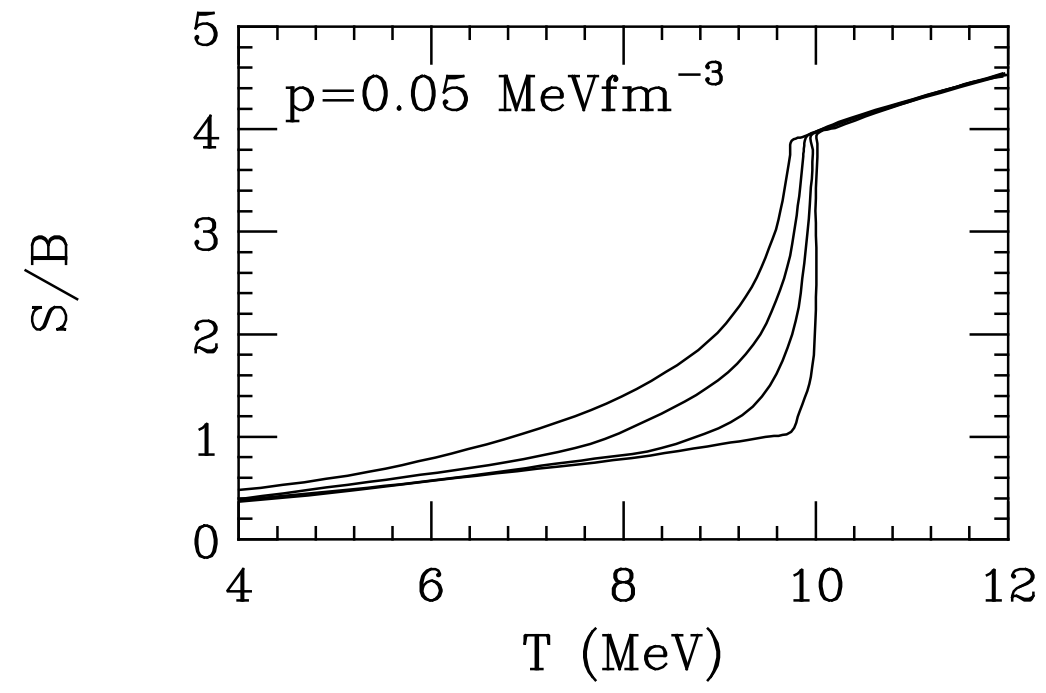

Figure 2.33: Specific entropy as a function of temperature at a constant pressure for proton fractions of $0.5,0.45,0.4$ and 0.35 , respectively. Taken from Ref. [16].

matter is the relative proton fraction in the two phases. Since the EOS of asymmetric matter contains a term $e_{\mathrm{sym}} \delta^{2}$, it is energetically favorable for the system to separate into a neutron-rich liquid phase and a proton-rich gas phase rather than two phases with equal proton concentration. The same conclusion has been reached from the study of chemical and dynamical instabilities in the previous section. However, one should be cautious in comparing this conclusion directly with experimental observations on the charge/mass ratio of heavy fragments and single particles or light clusters because the liquid-gas phase transition may occur in heavy residues during the later, expansion phase of nuclear reactions. In the latter case, the charge/mass ratio of the heavy-residue depends strongly on the symmetry potential during the early stage of the reaction. We shall discuss this in more detail in section 4.2 .

To confirm experimentally these new features of liquid-gas phase transition in asymmetric matter is challenging. One possible way is to study the dependence of nuclear multifragmentations on the charge/mass ratio of the reaction system. 


\section{Chapter 3}

\section{Isospin dependence of in-medium nucleon-nucleon cross sections}

In extracting information about the structure of radioactive nuclei, such as the radii and the distributions of the constituent neutrons and protons, the model used most is Glauber's. In the optical limit, the total interaction cross section is determined by a transmission function, which is a convolution of the nucleon-nucleon cross section and the density distribution of nucleons from both the target and projectile in the overlapping region. Knowing the proton density distribution (which can be determined from other means such as electron scattering) and the nucleon-nucleon cross sections, the neutron density distribution can then be determined. Usually, only the isospin averaged free nucleon-nucleon cross section is used as input. The effects of including the isospin-dependence of the in-medium nucleon-nucleon cross section needs to be studied. Also, the isospin-dependent in-medium nucleon-nucleon cross sections are needed in transport model to extract the isospin-dependent EOS and to understand the isospin-dependent phenomena found in heavy-ion collisions. In this chapter we will first discuss the isospin dependence of free-space nucleon-nucleon cross sections which are normally used as inputs in the isospin-dependent transport models. Then we shall review recent theoretical studies on the in-medium nucleon-nucleon cross sections based on the many-body theories discussed in the previous Chapter.

\subsection{Isospin dependence of free-space NN cross sec- tions}

It is well-known that the scattering cross section between two nucleons depends on their isospin. Fig. 3.1 compares the free-space cross sections for neutron-proton and proton-proton or neutron-neutron scattering as functions of bombarding energy. The data in the energy range of $10 \mathrm{MeV} \leq E_{\text {lab }} \leq 1000 \mathrm{MeV}$ can be parameterized by |111

$$
\begin{aligned}
& \sigma_{n p}^{\text {free }}=-70.67-18.18 \beta^{-1}+25.26 \beta^{-2}+113.85 \beta(\mathrm{mb}), \\
& \sigma_{p p}^{\text {free }}=13.73-15.04 \beta^{-1}+8.76 \beta^{-2}+68.67 \beta^{4}(\mathrm{mb}),
\end{aligned}
$$


where $\beta \equiv v / c$ is the velocity of the projectile nucleon. It is seen that below about

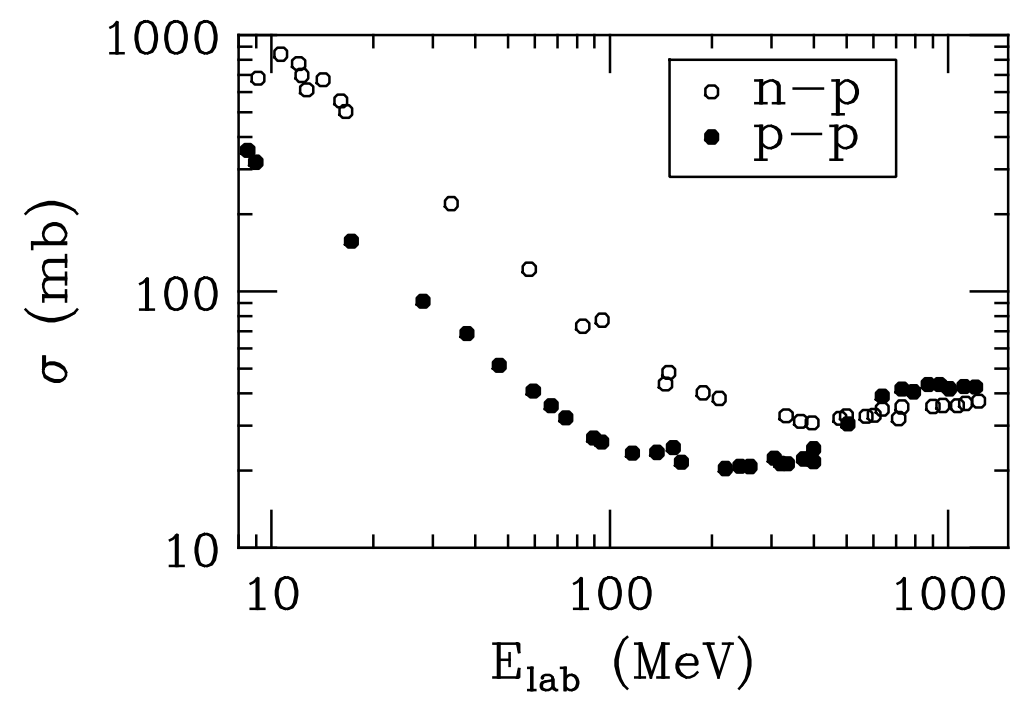

Figure 3.1: Cross sections of neutron-proton and proton-proton scatterings as functions of bombarding energy. Taken from Ref. [112].

$500 \mathrm{MeV}$ the neutron-proton cross section is about a factor of 2 to 3 higher than the proton-proton or neutron-neutron cross section, indicating that the $T$ matrix for the total isospin $I=0$ channel is much stronger than that of the $I=1$ channel. In nuclear medium, one expects that the strength of $G$ matrix, which differs from the free space $T$ matrix because of the Pauli effects and many-body effects, for these two channels are also different. How their relative strengths may change at finite density and temperature is a question of current interest. Since the reaction dynamics of heavy-ion collisions is governed by both the nuclear EOS and the in-medium nucleonnucleon cross sections, both are important and have to be studied simultaneously. In fact, the main uncertainty in the extracted nuclear EOS from heavy-ion collisions is due to our poor knowledge of the nucleon-nucleon cross sections in medium. It is thus desirable to find experimental observables that are only sensitive to either the EOS or the cross sections. This is particularly important for studying the symmetry potential since it is quite weak compared to the nuclear EOS of symmetric nuclear matter. As we shall discuss in the next chapter, transport models are very useful for such studies. 


\subsection{Isospin dependence of in-medium NN cross sections}

The study of in-medium nucleon-nucleon cross sections have been mostly based on the Bethe-Goldstone (BG) equation [22, 19, 113, 114, 115, 116, 117, 118, 119]. Medium effects appear in the BG equation mainly through the Pauli blocking factor for intermediate states and the self-energies of the two nucleons in the denominator of the propagator. However, results from these studies differ appreciably, with some models predicting a decrease of nucleon-nucleon cross sections while others an increase.

In the Dirac-Brueckner approach of Refs. [22, 117, model parameters are fixed by fitting free-space nucleon-nucleon scattering data and deuteron properties. Nucleonnucleon cross sections in nuclear medium at zero temperature have been predicted to decrease with increasing density. For example, at normal nuclear matter density and a bombarding energy of $50 \mathrm{MeV}$, both $\sigma_{n p}$ and $\sigma_{p p}$ are reduced by about a factor of two. Results of the calculations in Ref. [117] can be parameterized by

$$
\begin{aligned}
\sigma_{n p}^{\text {medium }} & =\left[31.5+0.092 a b s\left(20.2-E_{\mathrm{lab}}^{0.53}\right)^{2.9}\right] \cdot \frac{1.0+0.0034 E_{\mathrm{lab}}^{1.51} \rho^{2}}{1.0+21.55 \rho^{1.34}}(\mathrm{mb}), \\
\sigma_{p p}^{\text {medium }} & =\left[23.5+0.0256\left(18.2-E_{\mathrm{lab}}^{0.5}\right)^{4}\right] \cdot \frac{1.0+0.1667 E_{\mathrm{lab}}^{1.05} \rho^{3}}{1.0+9.704 \rho^{1.2}}(\mathrm{mb})
\end{aligned}
$$

Fig. 3.2 shows the neutron-proton and proton-proton cross sections at normal and twice normal nuclear matter density. One sees that the strong isospin dependence of nucleon-nucleon cross sections in free-space disappears gradually as the bombarding energy and density increase. Furthermore, at densities larger than about twice the normal density, the proton-proton cross section even becomes higher than the neutronproton cross section. The respective effects of the Pauli blocking and selfenergy corrections have not been studied in Ref. [117]. Bohnet et al. [114] have also studied the in-medium nucleon-nucleon cross sections during the collisions of two slabs of nuclear matter at zero temperature. The Pauli blocking factor has been estimated using two Fermi spheres separated by the beam momentum, and it is found that the in-medium cross section generally increases with density.

In the work by Alm et al. [118, 119, the cross section in a hot nuclear matter has been evaluated by including also the hole-hole collisions in the Pauli blocking operator. Fig. 3.3 shows their results at temperatures of $10 \mathrm{MeV}$ and $35 \mathrm{MeV}$ and a density of $0.5 \rho_{0}$ as a function of bombarding energy. Effects of the Pauli blocking and selfenergy corrections are separated by comparing full calculations with those by setting the Pauli blocking operator equals to 1 . First, it is seen that at both temperatures the selfenergy correction suppresses the cross section, while the Pauli blocking operator for intermediate states enhances the cross section. Second, at energies above about $200 \mathrm{MeV}$ predictions for different temperatures converge to values smaller than the free-space cross section. It is also seen that at lower temperatures the cross section 


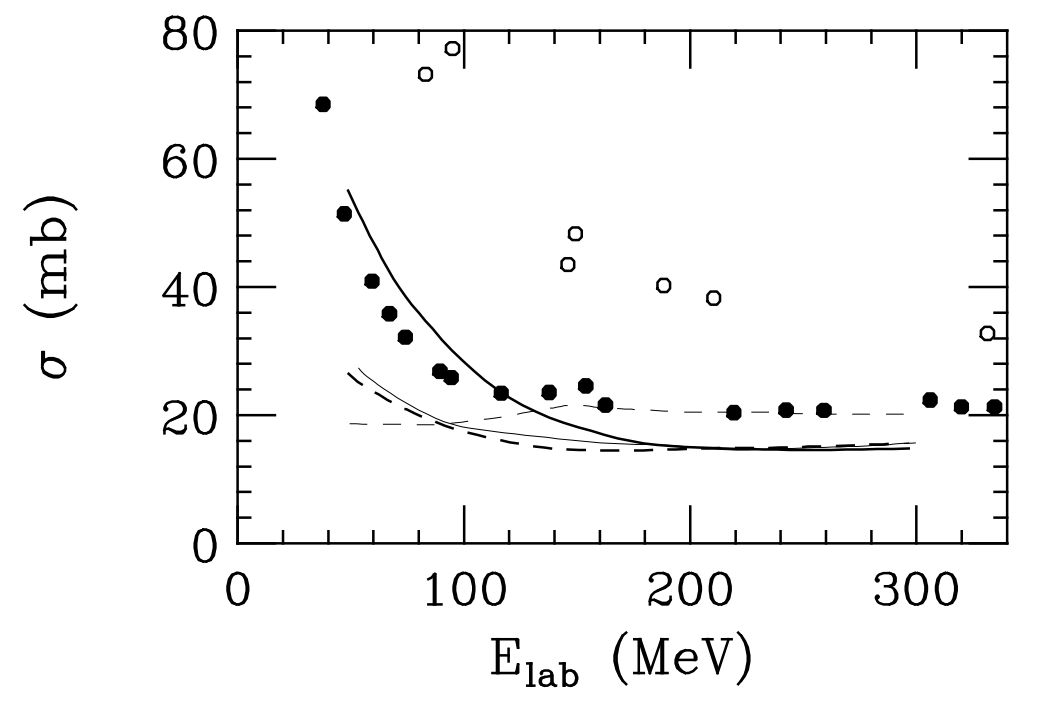

Figure 3.2: The neutron-proton (solid lines) and proton-proton (dashed lines) cross sections as functions of bombarding energy at normal (thick lines) and twice normal density (thin lines). The calculations are taken from Ref. 117 and are compared to the free cross sections of Fig. 3.1 (solid and open circles).

has a strong peak above the free-space cross section. This has been interpreted as a precursor effect of the superfluid phase transition in nuclear matter [118, 119].

In summary of this section, our current theoretical understanding about the inmedium nucleon-nucleon cross section, especially its dependence on isospin, density and temperature, is very limited. Most calculations are for nuclear matter only. Ideally, for heavy-ion collisions one should evaluate the in-medium cross sections selfconsistently at densities and temperatures determined by the reaction dynamics, and this requires much more work in the future. It is worth mentioning that recent studies on collective flow in heavy-ion collisions at intermediate energies have shown an indication of reduced in-medium nucleon-nucleon cross sections [120, 121, 122]. An empirical in-medium nucleon-nucleon cross section [121]

$$
\sigma_{N N}^{\text {medium }}=\left(1+\alpha \frac{\rho}{\rho_{0}}\right) \sigma_{N N}^{\text {free }}
$$

with the parameter $\alpha \approx 0.2$ has been found to better reproduce the flow data 120 , [122]. It is thus very promising that heavy-ion collisions may shed some light on the in-medium cross sections and their isospin dependence. 


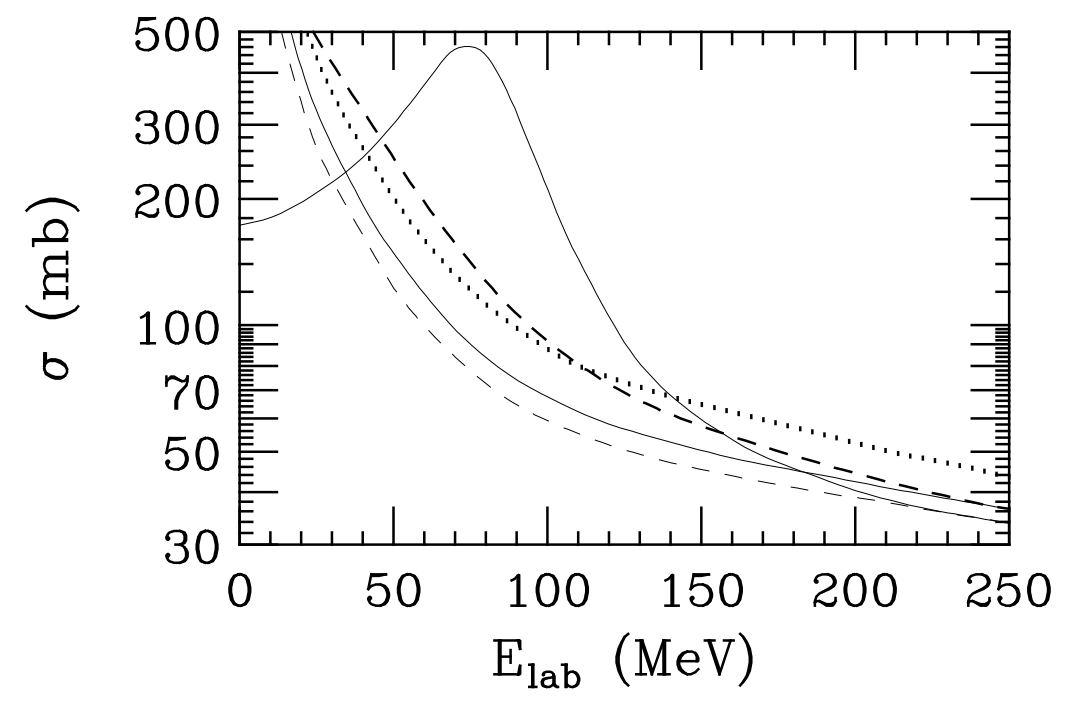

Figure 3.3: Isospin averaged nucleon-nucleon cross section as a function of bombarding energy at a density of $0.5 \rho_{0}$. Taken from Ref. [119]. The dotted line represents the free cross section, the solid lines represent the in-medium cross sections at temperatures of 10 (thin line) and $35 \mathrm{MeV}$ (thick line). The dashed lines are the corresponding cases without Pauli-blocking. 


\section{Chapter 4}

\section{Isospin-dependent phenomenology in heavy-ion collisions at intermediate energies}

Several new and interesting phenomena related to the isospin have been observed in heavy-ion collisions with radioactive beams 123. To understand such phenomena many existing models developed for heavy-ion collisions, such as the isospindependent percolation model [98], isospin-dependent lattice gas model [110] and isospin-dependent transport models, have been extended to include the isospin degree of freedom. We shall discuss in this chapter some of these phenomena and their explanations. Transport models are particularly useful for studying the isospin-dependent EOS and the in-medium nucleon-nucleon cross sections, and we shall therefore also discuss in this chapter the role of isospin in these models and their predictions.

\subsection{Isospin-dependent transport models for heavy- ion collisions}

A powerful framework to test various predictions on the isospin dependence of nuclear EOS and in-medium nucleon-nucleon cross sections is the nuclear transport theory. The isospin degree of freedom has been included in transport models at various levels of complexity [70, 71, 73, 74, 124, 125, 126, 127, 128, 129, 130. These models were developed from the well-known Boltzmann-Uehling-Uhlenbeck (BUU) transport theory [131, 132, 133, 134] or the Quantum Molecular Dynamics (QMD) theory [135]. by evolving separately the phase space distribution functions for protons and neutrons. In these models, the initial phase space distribution of nucleons in the target and projectile, nucleon-nucleon cross sections, mean-field potentials, and Pauli blocking are all isospin-dependent. For the isospin-dependent nucleon-nucleon cross section, the free-space cross sections shown in Fig. 3.1 have usually been used.

For the isospin-dependent mean-field potential, equations of state based on nuclear many-body theories discussed in section 2.2 have been used. For illustration we discuss here the properties of symmetry potential given in Eq. (2.39). For the simplest 
form of $F(u)$, i.e., $F(u)=F_{2}(u)=u$, one has

$$
V_{\text {asy }}^{n(p)}= \pm 2 e_{a} u \delta= \pm 2 e_{a} \frac{\rho_{n}-\rho_{p}}{\rho_{0}} .
$$

This is the asymmetric part of nuclear mean-field potential used in Refs. [73, 126, 129, 136, 137. The asymmetric energy density of Eq. (2.24) used in Refs. [70, 75] leads to the same mean-field potential at $\rho \approx \rho_{0}$ as Eq. (4.1). To illustrate the magnitude and variation of the symmetry potential, we show in Fig. 4.1 $V_{\text {asy }}^{n(p)}(\rho, \delta)$ using the three forms of $F(u)$ given in Eq. (2.19) and $e_{\text {sym }}\left(\rho_{0}\right)=32 \mathrm{MeV}$. It is seen that the

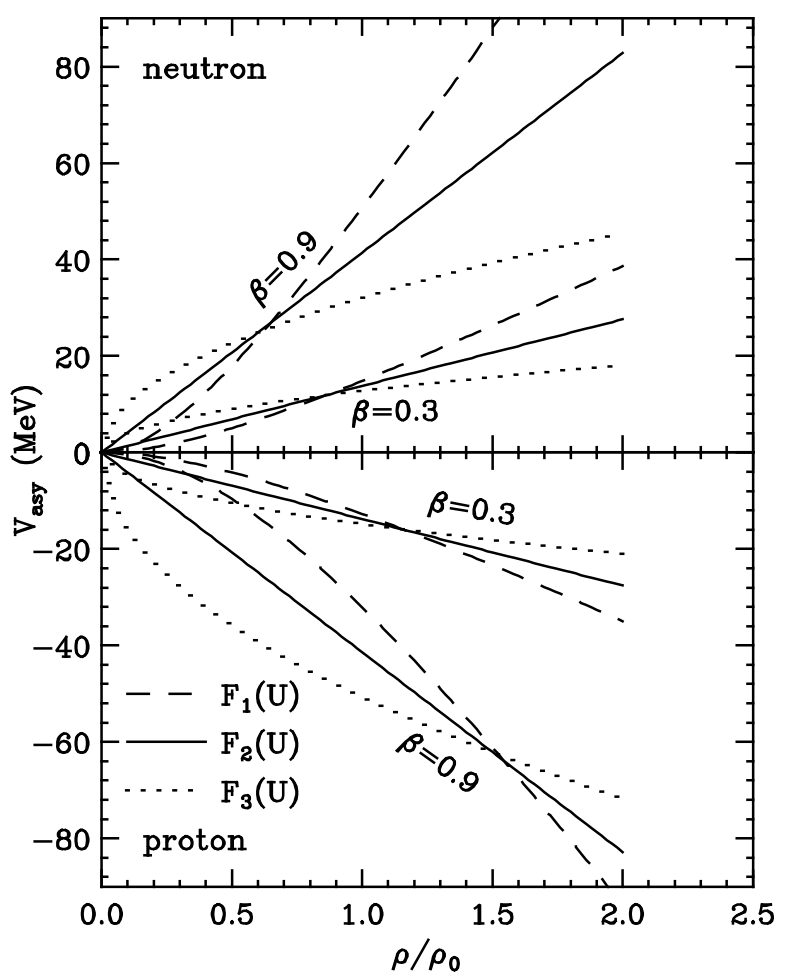

Figure 4.1: Symmetry potentials for neutrons and protons corresponding to the three forms of $F(u)$ (see text). Taken from Ref. [130].

repulsive (attractive) mean-field potential for neutrons (protons) depends sensitively on the form of $F(u)$, the neutron excess $\delta$, and the baryon density $\rho$. In collisions of neutron-rich nuclei at intermediate energies, both $\delta$ and $\rho$ can be appreciable in a large space-time volume where the isospin-dependent mean-field potentials, which are opposite in sign for neutrons and protons, are strong. This will affect differently 
the reaction dynamics of neutrons and protons, leading to possible differences in their yields and energy spectra.

In modeling heavy-ion collisions the nuclear mean-field potential should also include a Coulomb term $V_{C}^{p}$ for protons, i.e.,

$$
V^{n(p)}(\rho, \delta)=a\left(\rho / \rho_{0}\right)+b\left(\rho / \rho_{0}\right)^{\sigma}+V_{c}^{p}+V_{\text {asy }}^{n(p)}(\rho, \delta) .
$$

To extract $e_{\text {sym }}(\rho)$ from the experimental data requires observables that are sensitive to the asymmetric part but not the symmetric part of the nuclear EOS. This is necessary because the magnitude of $V_{\text {asy }}^{n(p)}$ is rather small compared to the symmetric part in Eq. (4.2). In addition, these observables should not depend strongly on other factors that affect the reaction dynamics, such as the in-medium nucleonnucleon cross sections. In this respect, it is interesting to mention that the possibility of extracting the isospin-dependent EOS from the elastic scatterings of neutron-rich nuclei has been examined recently by using a double-folding model [35, 138]. Both the isovector and isoscalar parts of the interaction potential between two neutron-rich nuclei are evaluated.

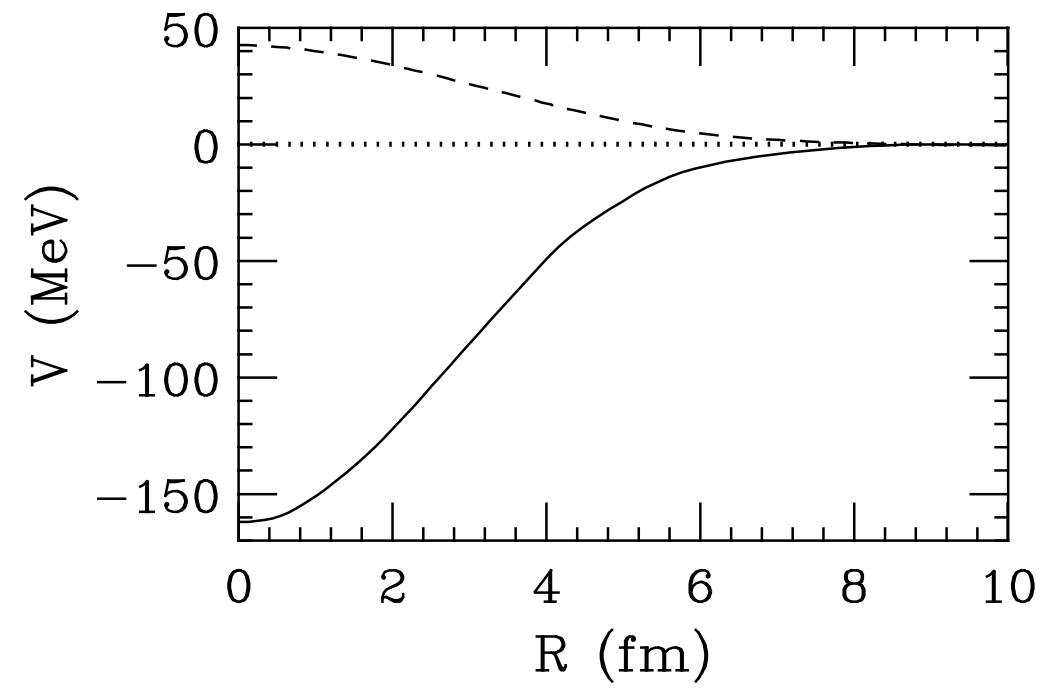

Figure 4.2: The symmetry and isoscalar potential for the scattering of ${ }^{11} \mathrm{Li}+{ }^{14} \mathrm{C}$ at a beam energy of $60 \mathrm{MeV} /$ nucleon. Shown is the calculation utilizing the BDM3Y1Paris potential. The dashed line represents the symmetry potential, and the solid line is the isoscalar potential. The results are taken from Ref. [35].

Shown in Fig. 4.2 are the isovector and isoscalar potentials for the scattering of ${ }^{11} \mathrm{Li}+{ }^{14} \mathrm{C}$ at a beam energy of $60 \mathrm{MeV} /$ nucleon. The strength of the symmetry potential (isovector) is much smaller than that of the isoscalar potential, and consequently the optical potential and thus the elastic scattering cross section is dominated by 
the isoscalar potential as shown in Fig. 4.3. As a result, it is difficult to extract the symmetry potential from elastic scattering data.

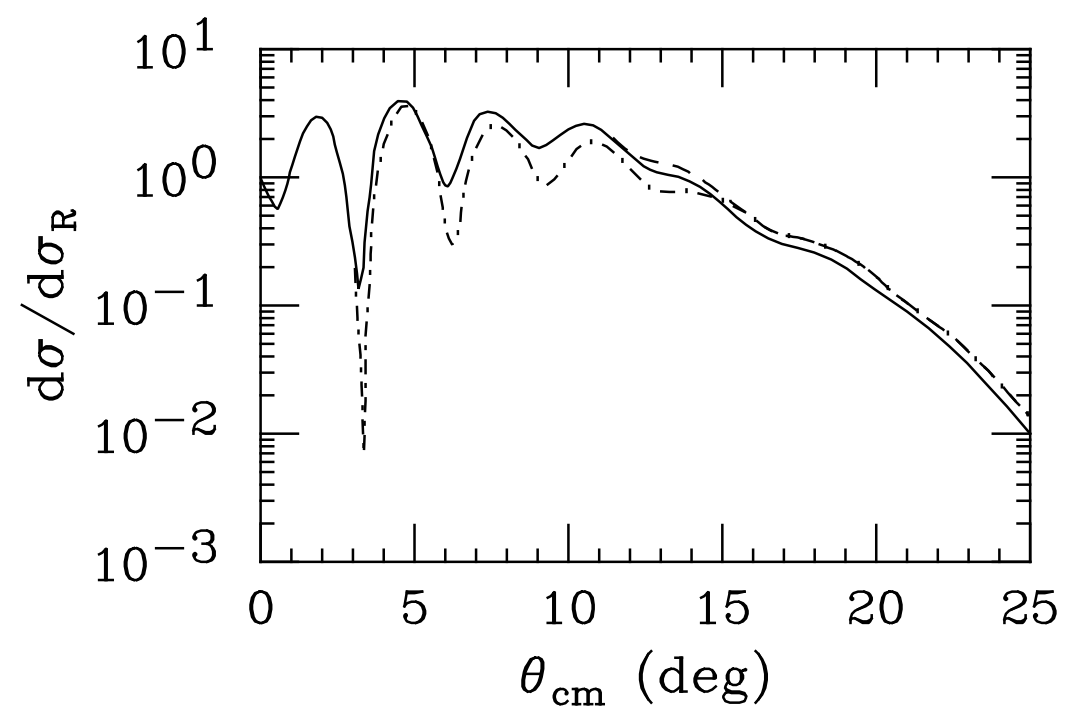

Figure 4.3: Elastic scattering cross section for ${ }^{11} \mathrm{Li}+{ }^{14} \mathrm{C}$ at a beam energy of 60 $\mathrm{MeV} /$ nucleon. Left window: the solid line is the result of a full calculation; the dashed line is obtained without the symmetry potential; and the dash-dotted line is obtained using the real potential folded with unrealistic compact density distribution for neutrons in ${ }^{11} \mathrm{Li}$. Taken from Ref. [35.

These studies further indicate the importance of selecting appropriate observables in order to learn the isospin-dependent part of the nuclear EOS. As in charge-exchange reactions, which are useful for extracting the isospin-dependent part of the nucleonnucleon interaction [139, 140, the ratio of preequilibrium neutrons to protons in heavy-ion collisions at intermediate energies have been found to be suitable for extracting the isospin-dependent EOS [91]. We shall discuss this ratio in detail in the next section using the isospin-dependent transport model of Refs. [74, 127, 128, 129, 130 .

To describe properly reactions induced by neutron-rich nuclei, it is important to use as realistic as possible initial neutron and proton distributions in the transport model. This can be achieved by initializing neutrons and protons randomly in a sphere with a radius corresponding to the rms radius of a heavy nucleus, as we have discussed in section 2.4. Then the BUU/LV model with a proper symmetry potential can describe the gross properties of the nucleus after evolving it in time, despite the fact that the model does not contain any information on its microscopic structure. Since the binding energy as well as density distributions of neutrons and protons depend on both the strength and form of symmetry potential, i.e., one obtains different density profiles using different symmetry potentials, it is thus possible to learn about the 
asymmetric EOS from studying saturation properties of neutron-rich nuclei. However, the information inferred from these studies is only around the saturation density. What is relevant for astrophysics, but poorly known, is the asymmetric EOS at other densities. These can only be reached in heavy-ion collisions. The effects of different symmetry potentials on particle emission in heavy ion collisions might be stronger than on equilibrium properties of neutron-rich nuclei. To observe these effects the same initial states for both the target and projectile have to be used in calculations using different symmetry potentials. In Refs. [129, 130], this has been achieved by using initial nucleon distributions given by the RMF theory.

\subsection{Isospin dependence of preequilibrium nucleon emission}

In this section, we will first discuss the ratio of preequilibrium neutrons to protons $\left(R_{n / p}\left(E_{\text {kin }}\right) \equiv d N_{n} / d N_{p}\right)$ from collisions of neutron-rich nuclei at intermediate energies by using the isospin-dependent BUU model. Then we will discuss experimental observations.

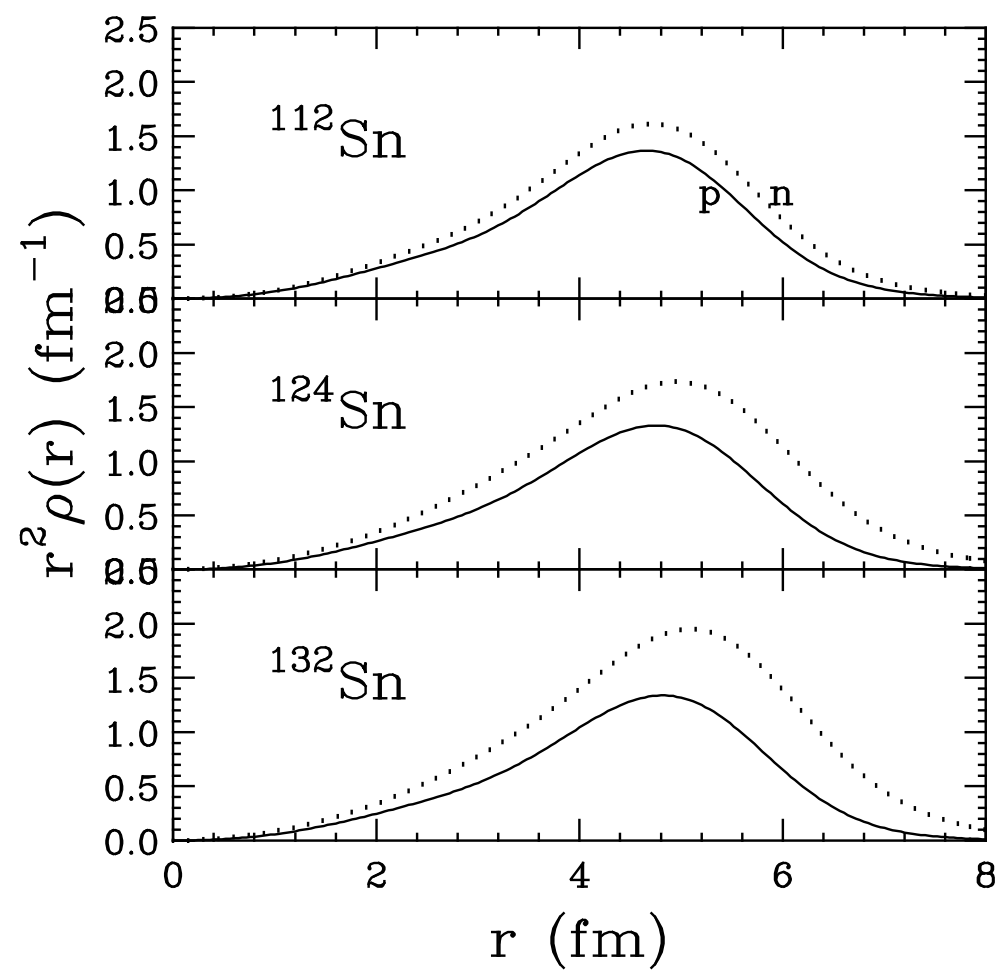

Figure 4.4: Density distributions of neutrons (dotted) and protons (solid) in Sn isotopes calculated using the RMF theory. 
As an example, collisions of ${ }^{112} \mathrm{Sn}+{ }^{112} \mathrm{Sn},{ }^{124} \mathrm{Sn}+{ }^{124} \mathrm{Sn}$ and ${ }^{132} \mathrm{Sn}+{ }^{132} \mathrm{Sn}$ at a beam energy of $40 \mathrm{MeV} /$ nucleon are studied. The first two reactions have been recently investigated experimentally at NSCL/MSU by the MSU-Rochester-WashingtonWisconsin collaboration [94, 95]. Preequilibrium particles have been measured in these experiments and are now being analyzed [141]. The last system is included for discussions and comparisons. The density distributions of neutrons and protons in the Sn isotopes are calculated using the RMF theory 142 and are shown in Fig. 4.4, and the local Thomas-Fermi approximation is then used to generate the nucleon momenta. To identify free nucleons, a phase-space coalescence method has been used at $200 \mathrm{fm} / \mathrm{c}$ after the initial contact of the two nuclei, when the quadrupole moment of the nucleon momentum distribution in the heavy residue is almost zero, indicating the approach of thermal equilibrium. A nucleon is considered as free if it is not correlated with other nucleons within a spatial distance of $\triangle r=3 \mathrm{fm}$ and a momentum distance of $\triangle p=300 \mathrm{MeV} / \mathrm{c}$. Otherwise, it is bounded in a cluster. These results are not sensitive to these parameters if they are varied by less than $30 \%$ around these values.

Effects of the compressibility $K$ of symmetric nuclear matter and the in-medium nucleon-nucleon cross sections on the ratio $R_{n / p}\left(E_{\text {kin }}\right)$ can be studied by neglecting both the Coulomb and symmetry potentials in the BUU model. In Fig. 4.5 this ratio is shown as a function of nucleon kinetic energy for central (upper window) and peripheral (lower window) collisions of ${ }^{132} \mathrm{Sn}+{ }^{132} \mathrm{Sn}$ at a beam energy of 40 $\mathrm{MeV} /$ nucleon. The total number of preequilibrium neutrons and protons and their ratios for the same reaction are shown in Fig. 4.6. When varying the compressibility $K$ from $210 \mathrm{MeV}$ (open squares) to $380 \mathrm{MeV}$ (filled circles), it is seen that although the yields of both protons and neutrons increase, their ratio remains similar for all impact parameters. This is simply because the effects of an isospin-symmetric EOS on neutrons and protons are identical.

The experimental neutron-proton cross section is about three times the neutronneutron (proton-proton) cross section in the energy range studied here. Setting the two cross sections equal (fancy squares), it has been found that the proton and neutron yields and their ratios change by less than $10 \%$ even in peripheral collisions of ${ }^{132} \mathrm{Sn}+{ }^{132} \mathrm{Sn}$. This result is also easy to understand since both colliding nucleons have the same probability to gain enough energy to become unbound [74]. Thus, the in-medium, isospin-dependent nucleon-nucleon cross sections do not affect much the ratio $R_{n / p}\left(E_{\text {kin }}\right)$. In the absence of Coulomb and symmetry potentials their ratio is thus almost independent of the nucleon kinetic energy and has a constant value of about $2.1 \pm 0.3$ in both central and peripheral collisions of ${ }^{132} \mathrm{Sn}+{ }^{132} \mathrm{Sn}$.

Including the Coulomb and the asymmetric term of the EOS in Eq. (4.2), one can then study the effects of the symmetry energy, $e_{\text {sym }}(\rho)$, since the Coulomb effect is well-known. The symmetry potential has the following effects on preequilibrium nucleons. First, it tends to make more neutrons than protons unbound. One therefore expects that a stronger symmetry potential leads to a larger ratio of free neutrons to protons. Second, if both neutrons and protons are already free, the symmetry potential makes neutrons more energetic than protons. These effects are clearly demonstrated in Fig. 4.7 where the ratios $R_{n / p}\left(E_{k i n}\right)$ are shown as a function of kinetic 


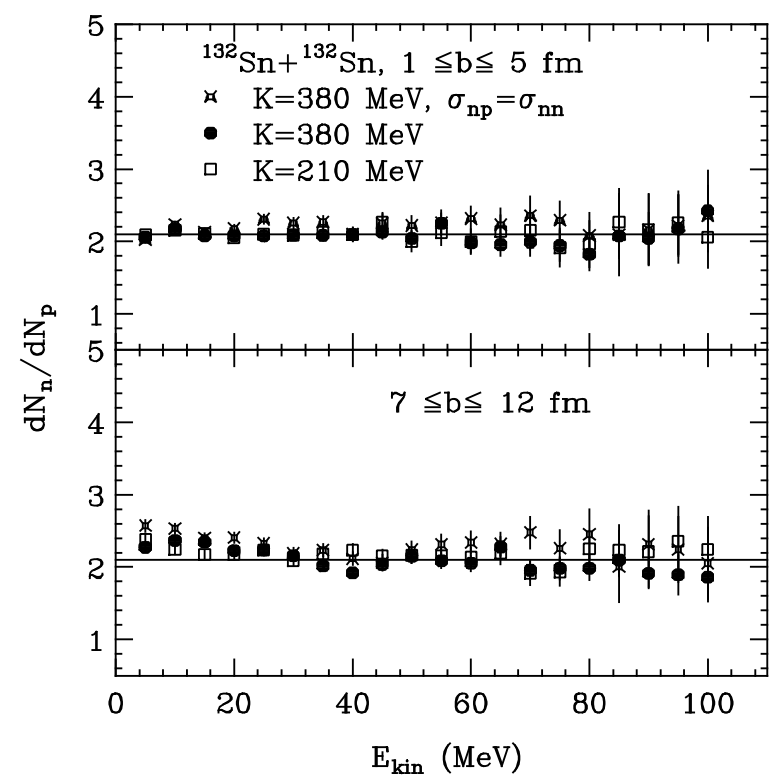

Figure 4.5: The ratio of preequilibrium neutrons to protons as a function of kinetic energy in central (upper window) and peripheral (lower window) reactions of ${ }^{132} \mathrm{Sn}+{ }^{132} \mathrm{Sn}$ at a beam energy of $40 \mathrm{MeV} /$ nucleon without the Coulomb and symmetry potentials. Taken from [91].

energy. These results are obtained by using the three forms of $F(u)$ for typical central (left windows) and peripheral (right windows) collisions of ${ }^{112} \mathrm{Sn}+{ }^{112} \mathrm{Sn},{ }^{124} \mathrm{Sn}+{ }^{124} \mathrm{Sn}$ and ${ }^{132} \mathrm{Sn}+{ }^{132} \mathrm{Sn}$, respectively. The increase of the ratios at lower kinetic energies in all cases is due to Coulomb repulsion which shifts protons from lower to higher kinetic energies. On the other hand, the different ratios calculated using different $F(u)$ 's reflect clearly the effect mentioned above, i.e., with a stronger symmetry potential the ratio of preequilibrium neutrons to protons becomes larger for more neutron rich systems. Between the central and peripheral collisions the ratios vary smoothly as a function of impact parameter. This is shown in Fig. 4.8 where the energy-dependence of the ratio $R_{n / p}\left(E_{\text {kin }}\right)$ is shown for different impact parameters for the reaction of ${ }^{124} \mathrm{Sn}+{ }^{124} \mathrm{Sn}$ using $F_{3}(u)$. The impact parameter dependence of the ratio of the total yield of neutrons to that of protons shown in Fig. 4.9 clearly demonstrates effects of the neutrons skins in more neutron-rich system at larger impact parameters.

It is interesting to note that effects due to different symmetry potentials are seen in different kinetic energy regions for central and peripheral collisions. This can be understood from the impact parameter dependence of $R_{n / p}\left(E_{\text {kin }}\right)$, shown in Fig. 4.8, and the reaction dynamics. In central collisions, effects of the symmetry potential are most prominent at higher kinetic energies. This is because most of finally observed free neutrons and protons are already unbound in the early stage of the reaction as a result of violent nucleon-nucleon collisions. The symmetry potential thus mainly affects the nucleon energy spectra by shifting more neutrons to higher 


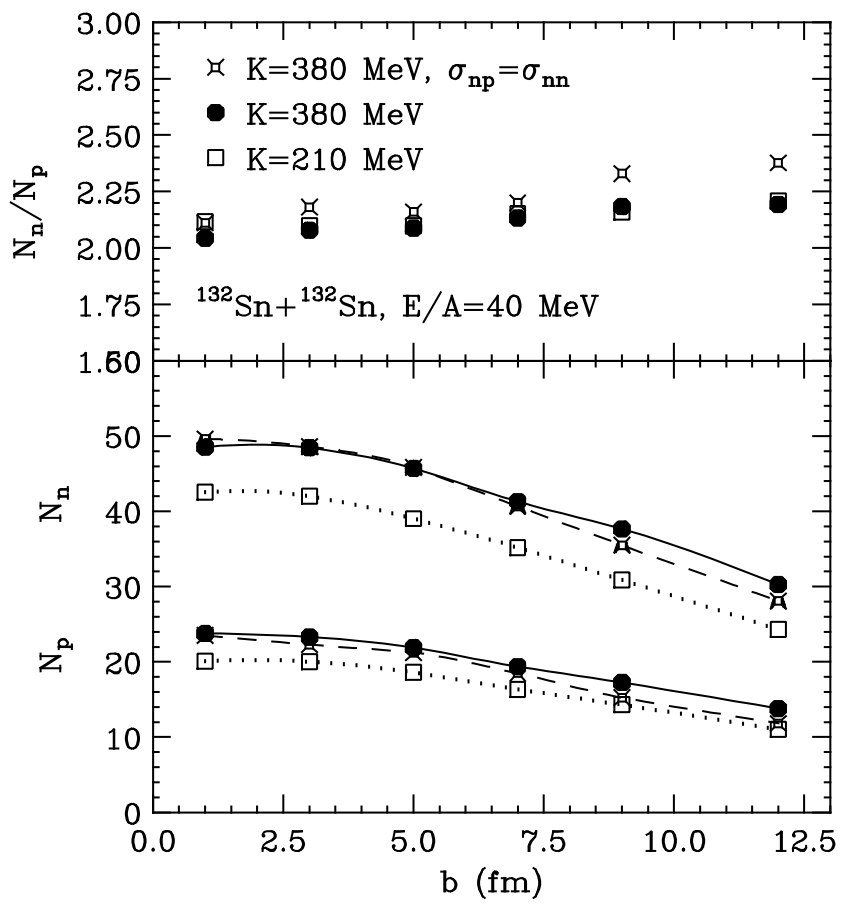

Figure 4.6: The total numbers of preequilibrium neutrons and protons (lower window) and their ratio (upper window) as functions of impact parameter for the reaction of ${ }^{132} \mathrm{Sn}+{ }^{132} \mathrm{Sn}$ at a beam energy of $40 \mathrm{MeV} /$ nucleon. 


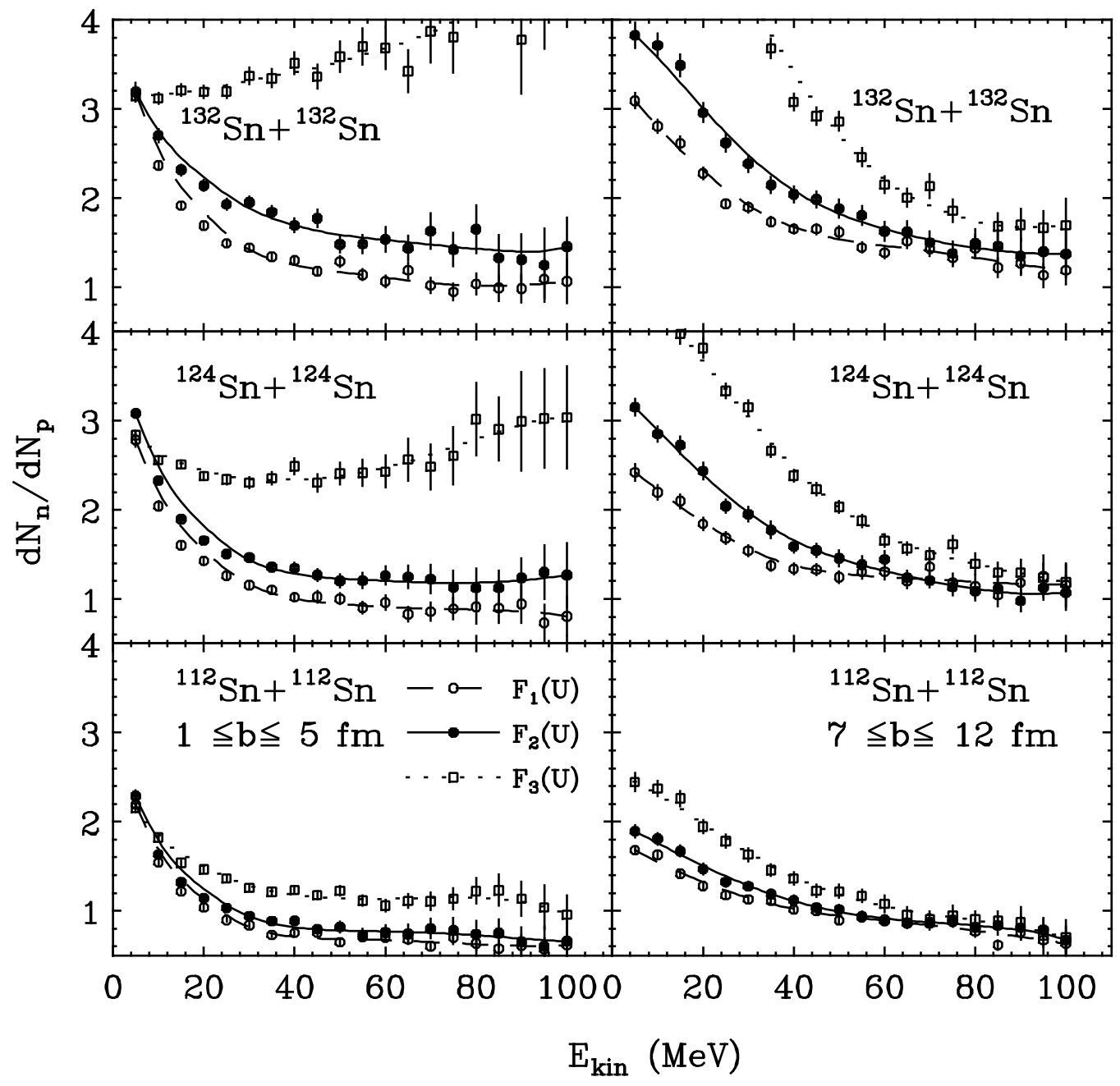

Figure 4.7: The ratio of preequilibrium neutrons to protons as a function of kinetic energy in central (left windows) and peripheral (right windows) reactions using the three forms of $F(u)$. Taken from [91]. 


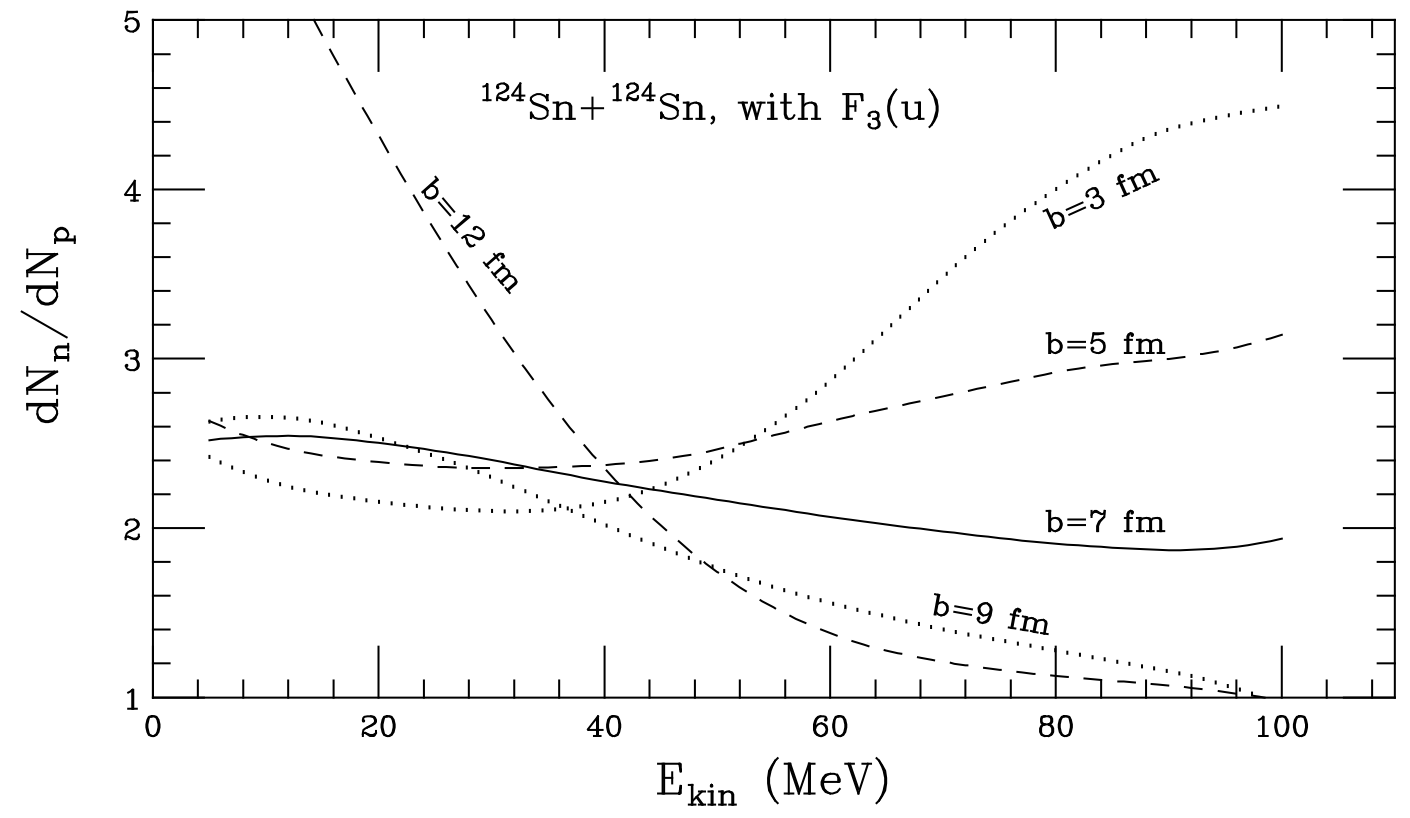

Figure 4.8: Impact parameter dependence of the ratio $R_{n / p}\left(E_{\text {kin }}\right)$ as a function of kinetic energy for the reaction of ${ }^{124} \mathrm{Sn}+{ }^{124} \mathrm{Sn}$ using $F_{3}(u)$.

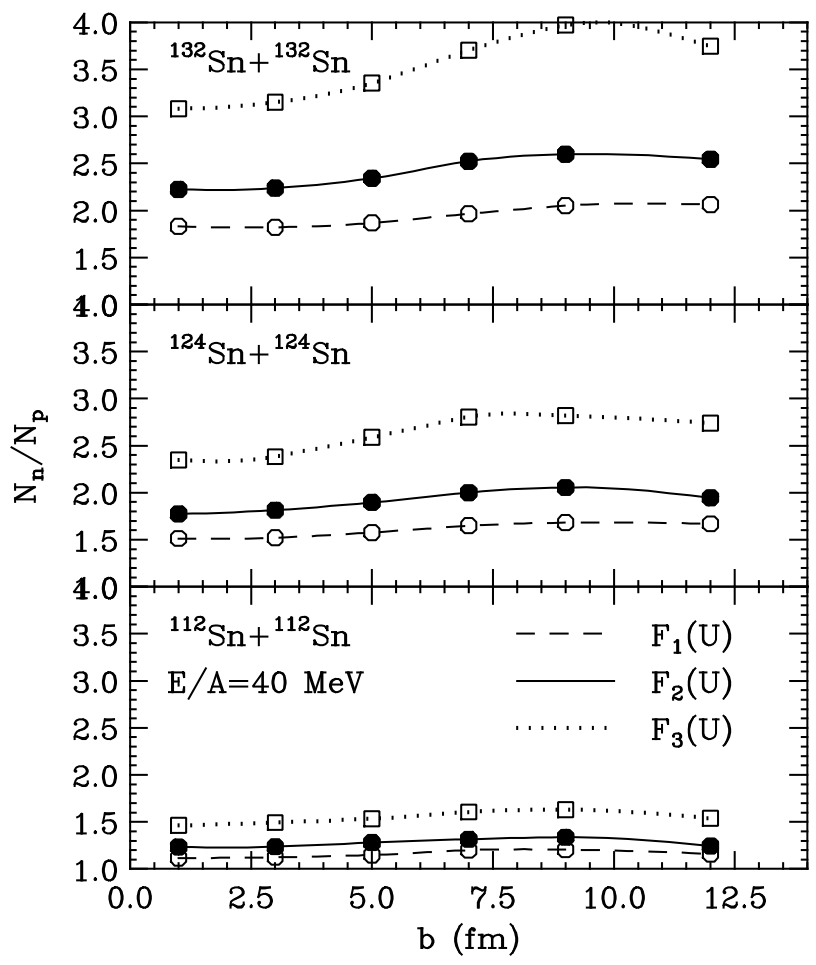

Figure 4.9: The ratio of preequilibrium neutrons to protons as a function of impact parameter using the three forms of $F(u)$. 
kinetic energies with respect to protons. In peripheral collisions, however, there are fewer nucleon-nucleon collisions; whether a nucleon can become unbound depends strongly on its potential energy. With a stronger symmetry potential more neutrons (protons) become unbound (bound) as a result of a stronger symmetry potential, but they generally have smaller kinetic energies. Therefore, in peripheral collisions effects of the symmetry potential show up chiefly at lower kinetic energies. For the two systems with more neutrons the effects of the symmetry potential are so strong that in central (peripheral) collisions different forms of $F(u)$ can be clearly distinguished from the ratio of preequilibrium neutrons to protons at higher (lower) kinetic energies. However, because of energy thresholds in detectors, it is difficult to measure low energy nucleons, especially neutrons. Furthermore, the low energy spectrum also has appreciable contribution from emissions at the later stage when the system is in equilibrium. Therefore, the measurement of the ratio $R_{n / p}\left(E_{\mathrm{kin}}\right)$ in central heavy-ion collisions for nucleons with energies higher than about $20 \mathrm{MeV}$ is more suitable for extracting the EOS of asymmetric nuclear matter. The beam energy range where

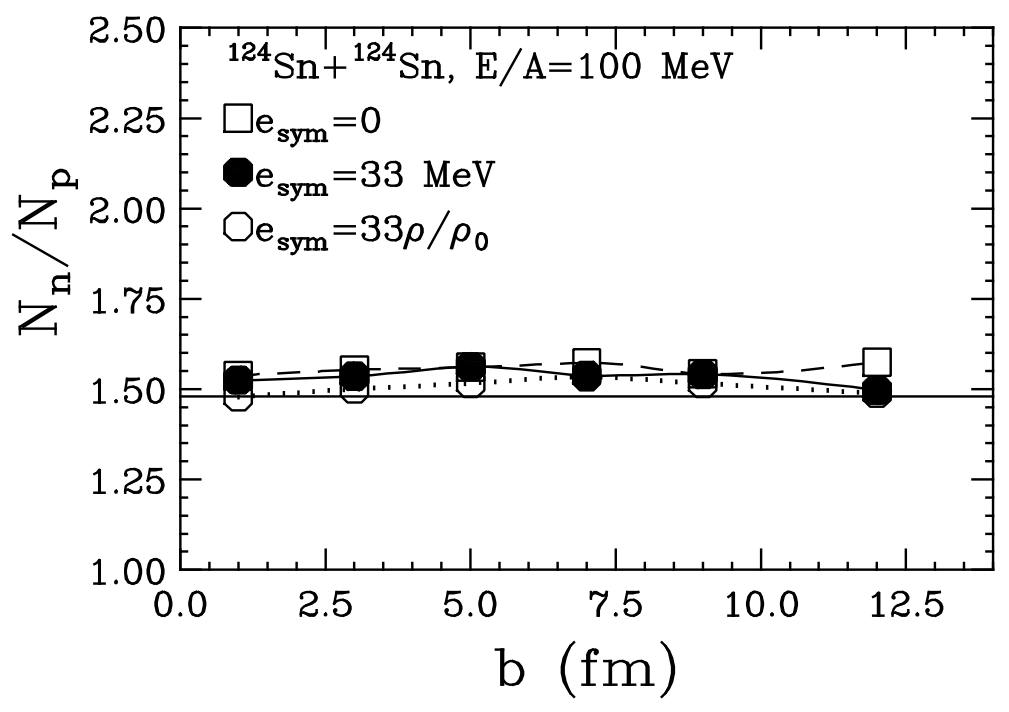

Figure 4.10: The ratio of preequilibrium neutrons to protons as a function of impact parameter for the reaction of ${ }^{124} \mathrm{Sn}+{ }^{124} \mathrm{Sn}$ at a beam energy of $100 \mathrm{MeV} /$ nucleon.

the symmetry potential is relevant for heavy-ion collisions depends on the isospin asymmetry of the reaction system and the observables to be studied. To observe effects of a weak mean-field potential, such as the symmetry potential, one needs to use relatively low beam energies so that the dynamics is not dominated by nucleonnucleon collisions. On the other hand, to study the density dependence and to reach a stronger mean-field potential the reactions should be energetic enough to achieve sufficient compression. Thus, it is necessary to study theoretically the beam energy dependence of the isospin effects on preequilibrium nucleons. Preliminary studies 
on the neutron/proton ratio in the reaction of ${ }^{124} \mathrm{Sn}+{ }^{124} \mathrm{Sn}$ indicate that the beam energy should not be greater than about $100 \mathrm{MeV}$. To illustrate this, we show in Fig. 4.10 the $R_{n / p}\left(E_{\text {kin }}\right)$ ratio as a function of impact parameter for this reaction at a beam energy of $100 \mathrm{MeV}$. Calculations corresponding to three typical cases of the symmetry energy, i.e., $e_{\text {sym }}=0,33 \mathrm{MeV}$, and $33 \rho / \rho_{0} \mathrm{MeV}$, have been carried out. It is seen that differences among results from these calculations are rather small and are all close to the $\mathrm{N} / \mathrm{Z}$ ratio of the reaction system, i.e., 1.48.

There seems already experimental evidence in heavy-ion collisions for the existence of a strong symmetry potential. For example, in experiments which measure preequilibrium nucleons, a number of puzzles have been observed, and they can be explained at least qualitatively by effects of the symmetry potential. In the following, several of these puzzles are discussed. In experiments of heavy-ion collisions around the Fermi energy [143, 144, 145, 146], Hilscher et al. have found that the neutron/proton ratio, $\left((N / Z)_{\text {free }}\right)$, of preequilibrium nucleons is consistently higher than that of the projectile-target system, $\left(N_{P}+N_{t}\right) /\left(Z_{p}+Z_{t}\right)$, and cannot be explained by the Coulomb effect. For example, in the reaction of ${ }^{12} \mathrm{C}+{ }^{165} \mathrm{Ho}$ at a beam energy of 32 $\mathrm{MeV} /$ nucleon, they have measured the neutron and proton spectra at $14^{\circ}$ and energies between 70 and $100 \mathrm{MeV}$, and found that the multiplicity of neutrons is larger than that of protons by a factor of $1.4 \pm 0.2,1.7 \pm 0.3$ and $2.4 \pm 0.3$ for linear-momentum transfers of $52 \%, 73 \%$ and $93 \%$, respectively. Therefore, the neutron to proton ratio is much higher than that of the reaction system $(N / Z)_{\mathrm{cs}}=1.42$ in central collisions corresponding to higher linear-momentum transfer, This result cannot be explained by the standard Fermi jet model for preequilibrium nucleon emission [143]. On the other hand, it is in agreement with the BUU predictions discussed above. From Fig. 4.7 and Fig. 4.9, it is seen that with a large symmetry potential the $\mathrm{n} / \mathrm{p}$ ratio for energetic nucleons can be much larger than that of the colliding system. Quantitative comparisons with the experiments will be very useful for extracting the isospin-dependent EOS. Semi-quantitative comparisons between theoretical results and some of the data have already been made using a simple symmetry potential energy density of Eq. (2.24) within the BUU/LV model [74, 75]. In Fig. 4.11 the relative neutron excess of preequilibrium nucleons $\delta_{\text {pre-eq }}=(N-Z) /(N+Z)$ is shown as a function of time for the reaction of ${ }^{12} \mathrm{C}+{ }^{165} \mathrm{Ho}$ at a beam energy of $35 \mathrm{MeV} /$ nucleon and an impact parameter of $1.0 \mathrm{fm}$. In the early stage of the reaction, the neutron excess is seen to fluctuate due to violent collisions, but reaches a constant value after about 100 $\mathrm{fm} / \mathrm{c}$. The dashed line at 0.17 is the neutron excess of the reaction system. Without the symmetry and Coulomb potential the final neutron excess of preequilibrium nucleons is about the same as that of the reaction system. In the case of including the Coulomb but not the symmetry potential $(c=0)$ more protons are emitted in the preequilibrium stage of the reaction due to the Coulomb repulsion. This effect has also been observed in other BUU calculations [70, 71, 73]. Furthermore, the ratio $(N / Z)_{\text {free }}$ increases monotonically as strength of the symmetry energy increases . To have a ratio larger than the $\mathrm{N} / \mathrm{Z}$ of the reaction system one needs to use a strength parameter $c \geq 20 \mathrm{MeV}$ corresponding to $e_{\mathrm{sym}}(0) \geq 32 \mathrm{MeV}$. More detailed comparisons with data are required to see what form and strength of the symmetry potential are needed to reproduce the data. 


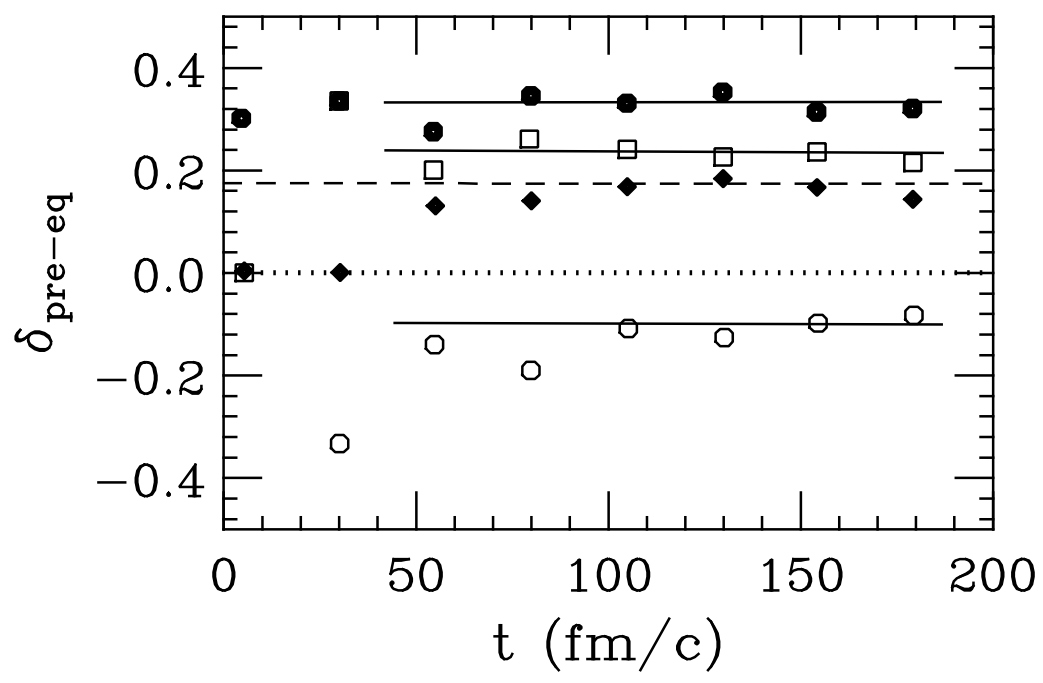

Figure 4.11: The ratio of preequilibrium neutrons to protons as a function of time for the reaction of ${ }^{12} \mathrm{C}+{ }^{165} \mathrm{Ho}$ at a beam energy of $35 \mathrm{MeV} /$ nucleon and an impact parameter of $1.0 \mathrm{fm}$. Solid circles: $c=46 \mathrm{MeV}$ with Coulomb; open squares: $c=32$ $\mathrm{MeV}$ with Coulomb; open circles: $c=0 \mathrm{MeV}$ with Coulomb; solid diamonds: $c=0$ $\mathrm{MeV}$ without Coulomb. Taken from [74]. 
A more quantitative comparison between the recent data from Ganil and the LV model calculation [75 has been carried out. In Fig. 4.12, the experimentally measured correlations between neutron multiplicities, $M_{N}$, in the forward direction $\left(6^{\circ} \leq \theta \leq 20^{\circ}\right)$ and fragment charges, $Z_{f}$, in the reaction of $\mathrm{Pb}+\mathrm{Au}$ at $E_{\text {beam }} / A=29$ $\mathrm{MeV}$ are shown as contours. The contour lines separating the areas in different shades (from white to dark gray) have the values $0.6,1,2$, and 3 , respectively. Calculations using the LV model without the symmetry potential $(c=0)$ are shown as open symbols and are seen to significantly underpredict the neutron multiplicities, especially in central collisions. In calculations with a symmetry energy strength parameter $c=20$ $\mathrm{MeV}$ (filled circles) the emission of neutrons is enhanced, so the data are better described.

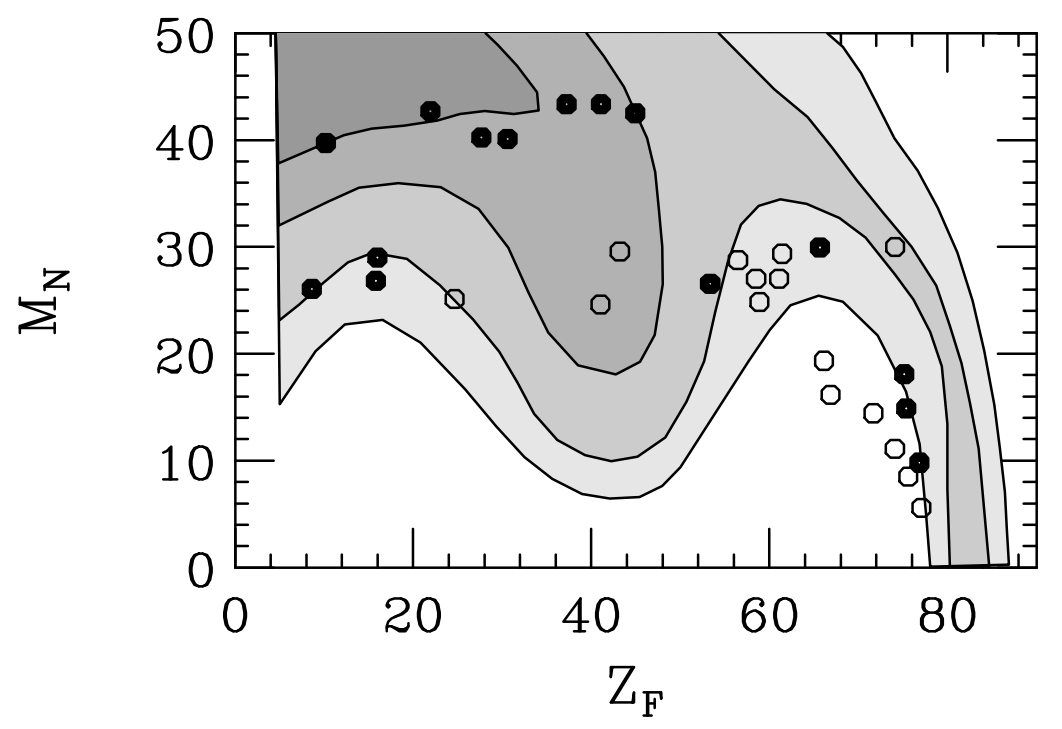

Figure 4.12: Experimental neutron multiplicity versus fragment charge for peripheral reactions of $\mathrm{Pb}+\mathrm{Au}$ at a beam energy of $29 \mathrm{MeV} /$ nucleon. LV model calculations with (without) the symmetry potential are given by filled (open) symbols. Taken from [75].

Another interesting experimental observation is the ratio of free neutrons and protons from central reactions of ${ }^{32} \mathrm{~S}+{ }^{144,154} \mathrm{Sm} \rightarrow F, F+n, p$, where $F$ denotes nuclear fragments, at $E_{\text {beam }} / A=26 \mathrm{MeV}$ as shown in Fig. 4.13. We note that protons emitted at velocities higher than the projectile velocity are mainly preequilibrium particles. Several interesting observations can be made from these data. First, the emission of protons in the neutron-richer system $\left(\mathrm{S}+{ }^{154} \mathrm{Sm}\right)$ is suppressed although both systems have the same number of protons to begin with. As we have discussed in detail in the previous section, protons feel an attractive symmetry potential, and the emission of high energy protons is thus suppressed with respect to neutrons. Correspondingly, the emission of high energy neutrons is enhanced, and this is indeed consistent with the 
neutron data at higher kinetic energies. Second, even for neutrons with lower kinetic energies the ratio of free neutrons from the two systems is about 1.23 and is much larger than the ratio (1.12) of neutrons of the two systems. More recently, Schröder et al. have studied systematically the spectra of preequilibrium neutrons and protons in both isospin symmetric and asymmetric collisions [147. It is found that the $\mathrm{n} / \mathrm{p}$ ratio of preequilibrium nucleons does not scale with the $\mathrm{N} / \mathrm{Z}$ ratio of the combined system. In particular, a preliminary analysis of the experimental data indicates that the ratio $(n / p){ }^{48} \mathrm{Ca}+{ }^{112} \mathrm{Sn} /(n / p){ }^{40} \mathrm{Ca}+{ }^{112} \mathrm{Sn}$ at a beam energy of $35 \mathrm{MeV} /$ nucleon is about 4 to 12 depending on the impact parameter. A similar observation has been made by Hilscher et al.. Both results are thus consistent with the existence of a strong symmetry potential.

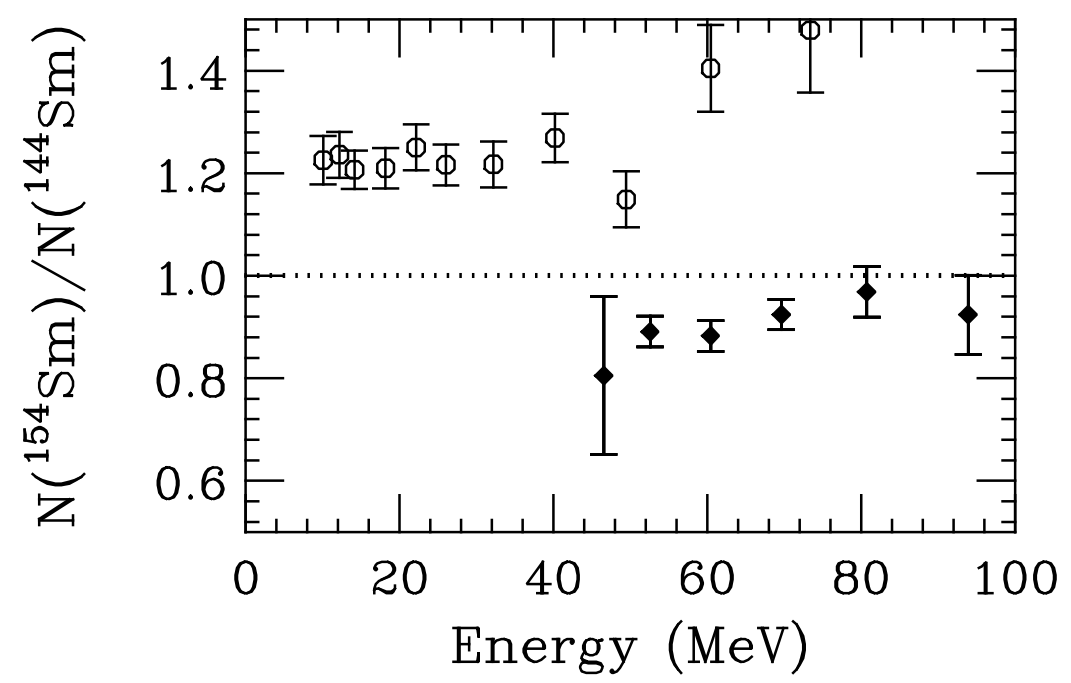

Figure 4.13: The ratio of free neutrons (open circles) and protons (solid diamond) in central reactions of ${ }^{32} \mathrm{~S}+{ }^{144,154} \mathrm{Sm}$ as a function of the kinetic energy of the emitted particle, at a beam energy of $26 \mathrm{MeV} /$ nucleon. Taken from [144].

Enhanced production of free neutrons than that predicted by the standard statistical model have also been reported in Refs. [94, 148]. In Ref. [149] it was pointed out that within the experimental and model uncertainties the production of neutrondeficient residues is much higher than the statistical model predictions. Because of charge conservation, this result is thus equivalent to an enhanced neutron emission as shown in Refs. 995, 148]. This is again consistent with the existence of a strong symmetry potential. To extract quantitative information about the nuclear symmetry potential requires, however, more detailed studies using the transport model.

To summarize, we have shown that in the isospin-dependent BUU model for heavyion collisions at intermediate energies the calculated ratio of preequilibrium neutrons to protons is very sensitive to the symmetry potential. Longstanding puzzles observed 
in preequilibrium neutrons and protons can be understood qualitatively by considering effects of the symmetry potential. More quantitative comparisons with data are still needed to constrain the form and strength of the symmetry potential.

\subsection{Isospin equilibrium as a probe of nuclear stop- ping power}

The degree of stopping in heavy-ion collisions is an important quantity in determining the outcome of a reaction. It is usually studied by measuring the rapidity distribution or the asymmetry of the nucleon momentum distribution. If we were able to tag the nucleons from projectile and target, then this task would be much easier. By using nuclei with different $\mathrm{N} / \mathrm{Z}$ ratios, such a tag can be provided. Recently, it has thus been suggested that the degree of equilibration in the isospin degree of freedom also provides a means to probe nuclear stopping in heavy ion collisions [150], as illustrated in Fig. 4.14.

The pictures on the left show the nucleon distributions in coordinate space, while that on the right are the rapidity distributions for the three scenarios of pile-up and bounce-back, stopping and mixing (isospin equilibrium), and transparency. It is seen that for a projectile and a target with very different $\mathrm{N} / \mathrm{Z}$ ratios, a comparison of the rapidity distribution of $\mathrm{N} / \mathrm{Z}$ before (shown in top picture on the right) and after the collision can give direct information about the degree of stopping between the target and projectile [129, 150]. This method thus allows one to study whether there is a transition from full stopping to transparency as the beam energy varies. Also, if isospin equilibrium can be reached in the collisions, it is then possible to determine its time scale relative to that for thermal equilibrium. Moreover, one can study the dependence of isospin equilibrium on the nuclear EOS and the in-medium nucleonnucleon cross sections. We note that this method is restricted to beam energies that are below the pion production threshold; so the total nucleon charge is conserved.

These ideas have been studied in the isospin-dependent QMD model under various

conditions as shown in Fig. 4.15. In the left window central collisions of ${ }^{50} \mathrm{Cr}+{ }^{48} \mathrm{Ca}$ and ${ }^{50} \mathrm{Cr}+{ }^{50} \mathrm{Cr}$ at $E_{\text {beam }} / A=1.0 \mathrm{GeV}$ are compared, and it shows clearly that there is a transparency in the asymmetric system. In the right window two calculations for the asymmetric system for different beam energies are shown, and it is seen that even at $150 \mathrm{MeV} /$ nucleon the asymmetric system shows transparency. Bass et al. further show that the signature for transparency seen in the N/Z ratio is not altered by cluster formation. On the other hand, the stopping power is affected by the magnitude of the nucleon-nucleon cross section. As expected, increasing the in-medium nucleonnucleon cross section by a factor of 5 results in a transition from transparency to full stopping.

The idea of using isospin to study the nature of stopping in nuclear collisions has been introduced earlier in Ref. [129] to explain the angular distribution of isotope ratios observed in experiments by Yennello et al. [151, 152, 153]. In these experiments the isotopic composition of intermediate mass fragments from central collisions of 


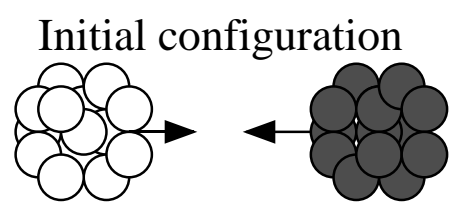

Pile-up/bounce-back

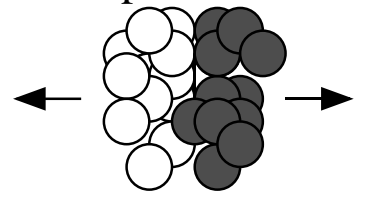

Complete equilibration

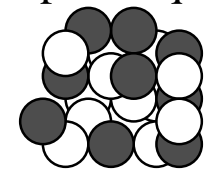

Partial transparency

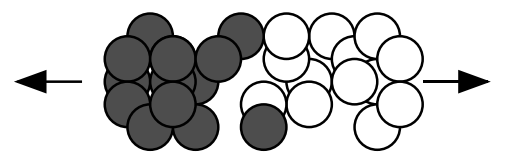

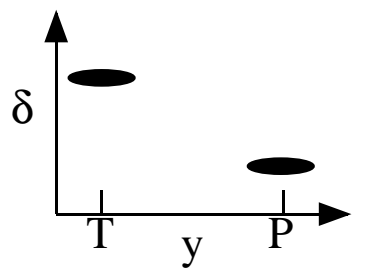
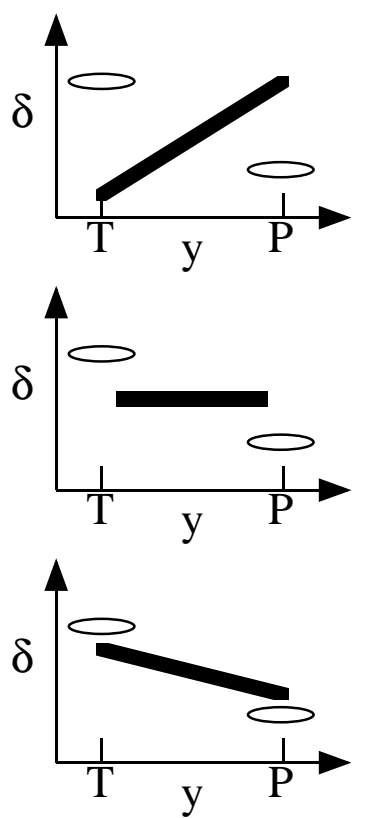

Figure 4.14: Illustration of using the rapidity distribution of neutron/proton ratio as a probe of nuclear stopping power. (A similar illustration can be found in Ref. [150].) 


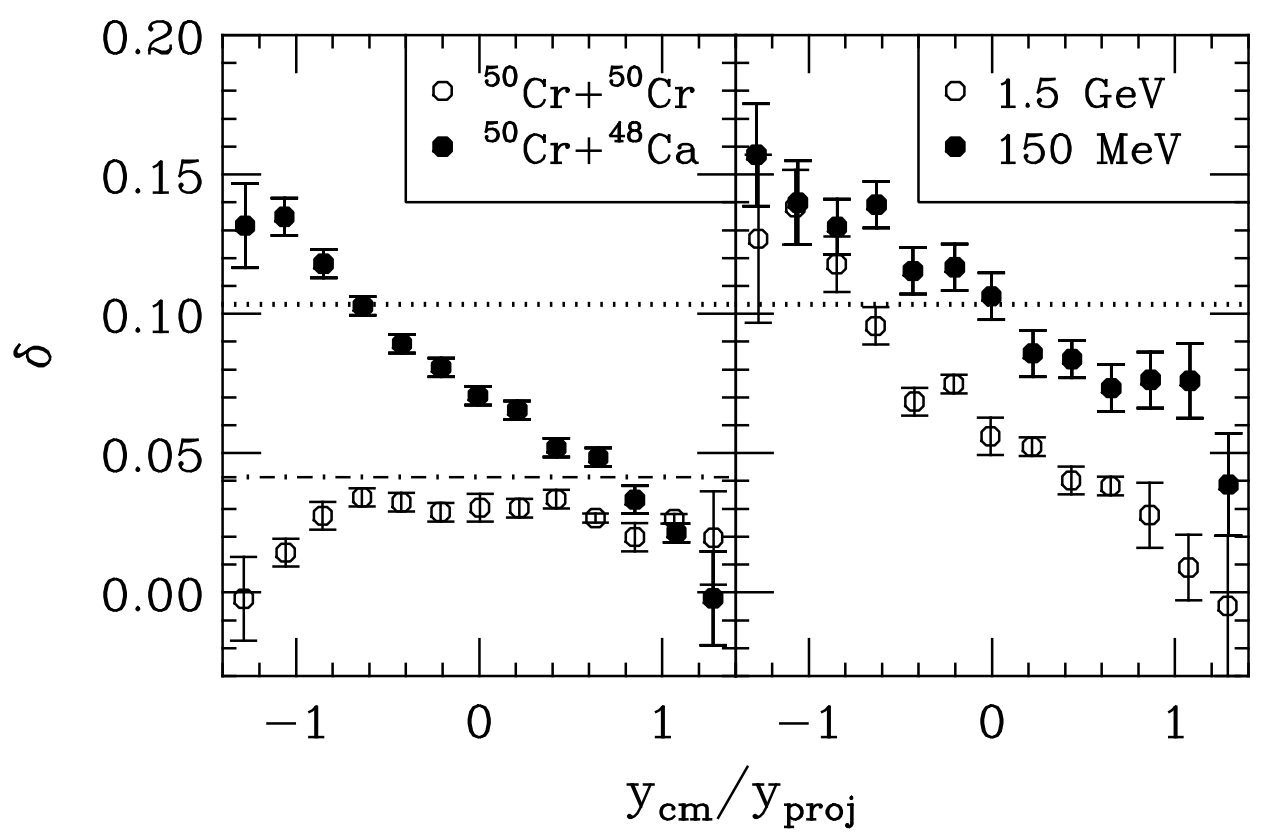

Figure 4.15: The neutron to proton asymmetry versus rapidity predicted by the QMD model. Taken from Ref. [150].

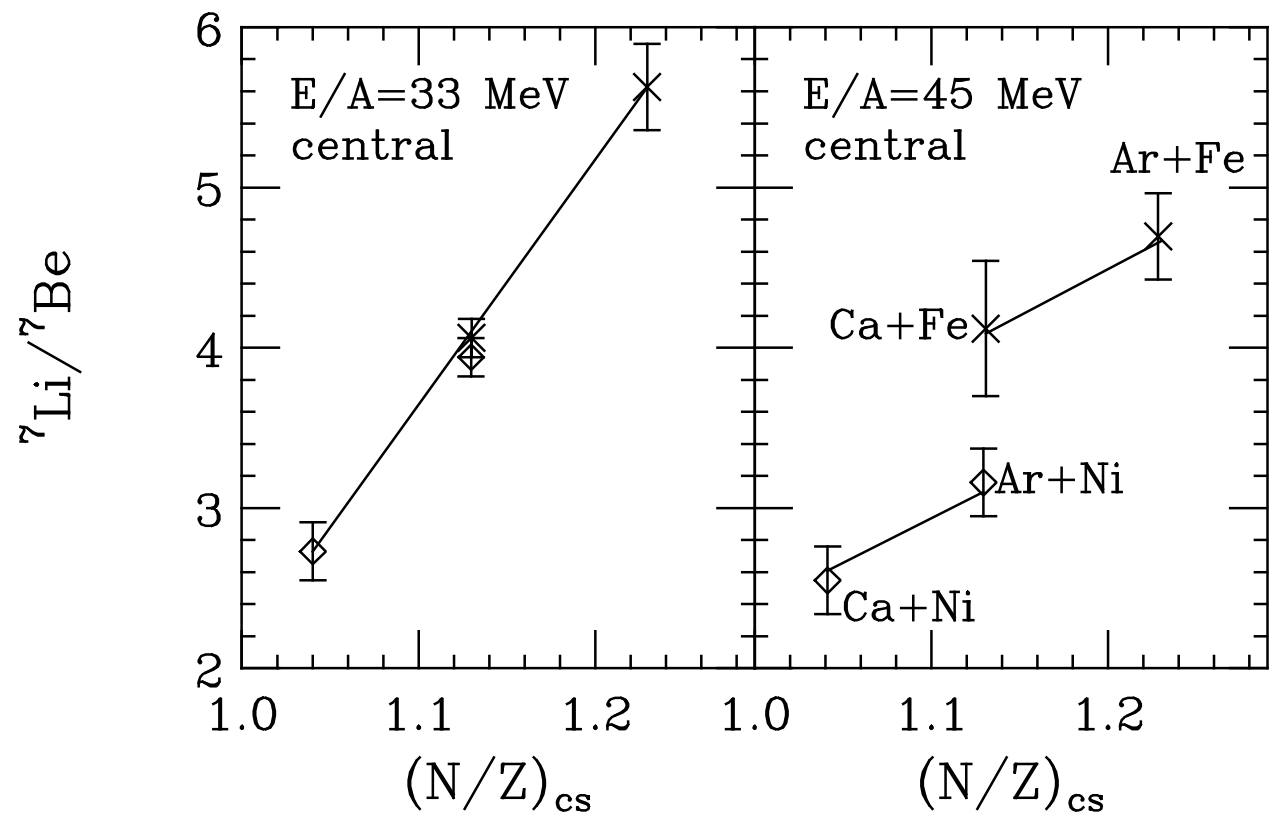

Figure 4.16: Isobaric ratios from central collisions plotted as a function of $\mathrm{N} / \mathrm{Z}$ ratio of the combined target and projectile system at $E_{\text {beam }} / A=35$ and $45 \mathrm{MeV}$. Taken from Ref. [153. 
${ }^{40} \mathrm{Cl},{ }^{40} \mathrm{Ar}$ and ${ }^{40} \mathrm{Ca}$ with ${ }^{58} \mathrm{Fe}$ and ${ }^{58} \mathrm{Ni}$ at $E_{\text {beam }} / A=25,35,45$ and $53 \mathrm{MeV}$ was studied at NSCL/MSU and TAMU. It was found that at $E_{\text {beam }} / A=25$ and $33 \mathrm{MeV}$ the isotopic ratios ${ }^{9} \mathrm{Be} /{ }^{7} \mathrm{Be},{ }^{11} \mathrm{~B} /{ }^{10} \mathrm{~B}$ and ${ }^{13} \mathrm{C} /{ }^{12} \mathrm{C}$ increase linearly with increasing $(N / Z)_{\text {cs }}$ ratio of the combined target and projectile system, but are independent of the N/Z ratio of the target or projectile. Shown in the left window of Fig. 4.16 are typical results of reactions at $33 \mathrm{MeV} /$ nucleon, which indicate that the isospin is equilibrated in the composite system formed in these reactions before the emission of fragments.

The most striking and unexpected feature was observed from the isotopic ratios in central collisions at $E_{\text {beam }} / A=45$ and $53 \mathrm{MeV}$. A typical result at $E_{\text {beam }} / A=45$ $\mathrm{MeV}$ is shown in the right window of Fig. 4.16. It is seen that the isotopic ratios depend on the $\mathrm{N} / \mathrm{Z}$ ratio of the target and projectile in reactions with target-projectile combinations having the same $(N / Z)_{C S}$ ratio, such as ${ }^{40} \mathrm{Ca}+{ }^{58} \mathrm{Fe}$ and ${ }^{40} \mathrm{Ar}+{ }^{58} \mathrm{Ni}$. In ref. [153], similar results are also shown for other isotope ratios.

Moreover, data at very forward and backward angles show that the isotope ratios do not simply depend on $(N / Z)_{\mathrm{cs}}$. Instead, light fragments at backward angles are seen to have a much stronger dependence on $(N / Z)_{\text {target }}$, while at forward angles they

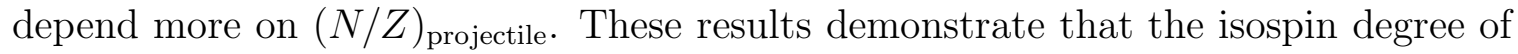
freedom in reactions at $E_{\text {beam }} / A=45$ and $53 \mathrm{MeV}$ is not equilibrated during the time when fragments are emitted. Therefore, a transition from isospin equilibration to non-equilibration is observed as the beam energy increases from below to above the Fermi energy.

The above observation has profound implications on the reaction mechanism leading to multifragmentation. It not only establishes the relative time scale for multifragmentation in these reactions but also indicates that the assumption of isospin equilibrium taken for granted in various statistical models for nuclear multifragmentation at intermediate energies is not valid. Indeed, a statistical model study was made and failed to show any entrance channel effect [151]. Calculations using an intranuclear cascade code ISABEL [155] show that the N/Z ratio of the residue is very close to that of the initial combined system [151] and thus also fails to reproduce those features observed at $E_{\text {beam }} / A=45$ and $53 \mathrm{MeV}$. Although the exact origin of this failure is not clear, one expects that the reaction dynamics at these relatively low energies cannot be described by the nucleon-nucleon cascade alone, and should include also nuclear mean-field potential.

The experimental observation discussed above can be explained by the isospindependent BUU model outlined in section 4.1. Calculations based on this model have been performed over a range of impact parameters. For peripheral collisions it shows a memory of the initial target and projectile, which is, however, gradually lost as the collisions become more central. A calculation at $b=0$ thus gives the most interesting test of any non-equilibrium effect. In Fig. 4.17, we show the energy dependence of the density contours in the reaction plane at $\mathrm{t}=200 \mathrm{fm} / \mathrm{c}$ in head-on collisions of $\mathrm{Ar}+\mathrm{Ni}$ at $E_{\text {beam }} / A=25,35,45$ and $55 \mathrm{MeV}$. The solid contours with $\rho=\rho_{0} / 8$ correspond essentially to bound composite systems or heavy residues formed in the reactions. The dotted contours with $\rho=0.05 \rho_{0}$ are free nucleons emitted mainly before thermal equilibrium is reached. The most interesting feature in Fig. 4.17 is the 


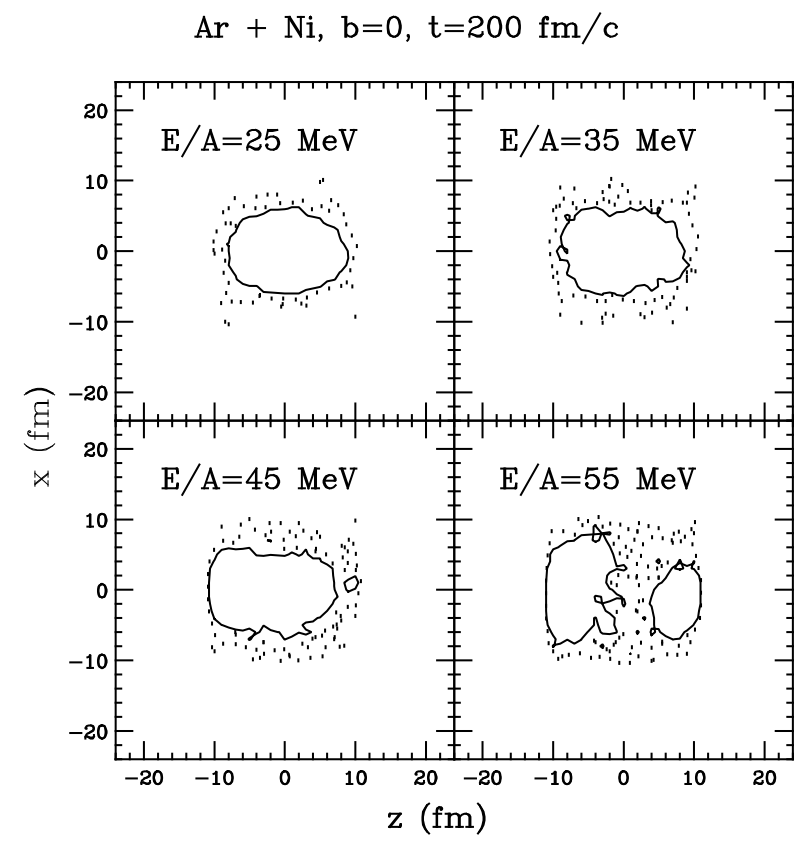

Figure 4.17: Density contours in the reaction plane at $\mathrm{t}=200 \mathrm{fm} / \mathrm{c}$ in head-on collisions of $\mathrm{Ar}+\mathrm{Ni}$ at $E_{\text {beam }} / A=25,35,45$ and $55 \mathrm{MeV}$. Taken from Ref. 129. 
formation of two heavy residues in the reaction at $E_{\text {beam }} / A=55 \mathrm{MeV}$. For reactions at $E_{\text {beam }} / A=25,35$ and $45 \mathrm{MeV}$, these heavy residues do not break up even after $300 \mathrm{fm} / \mathrm{c}$.

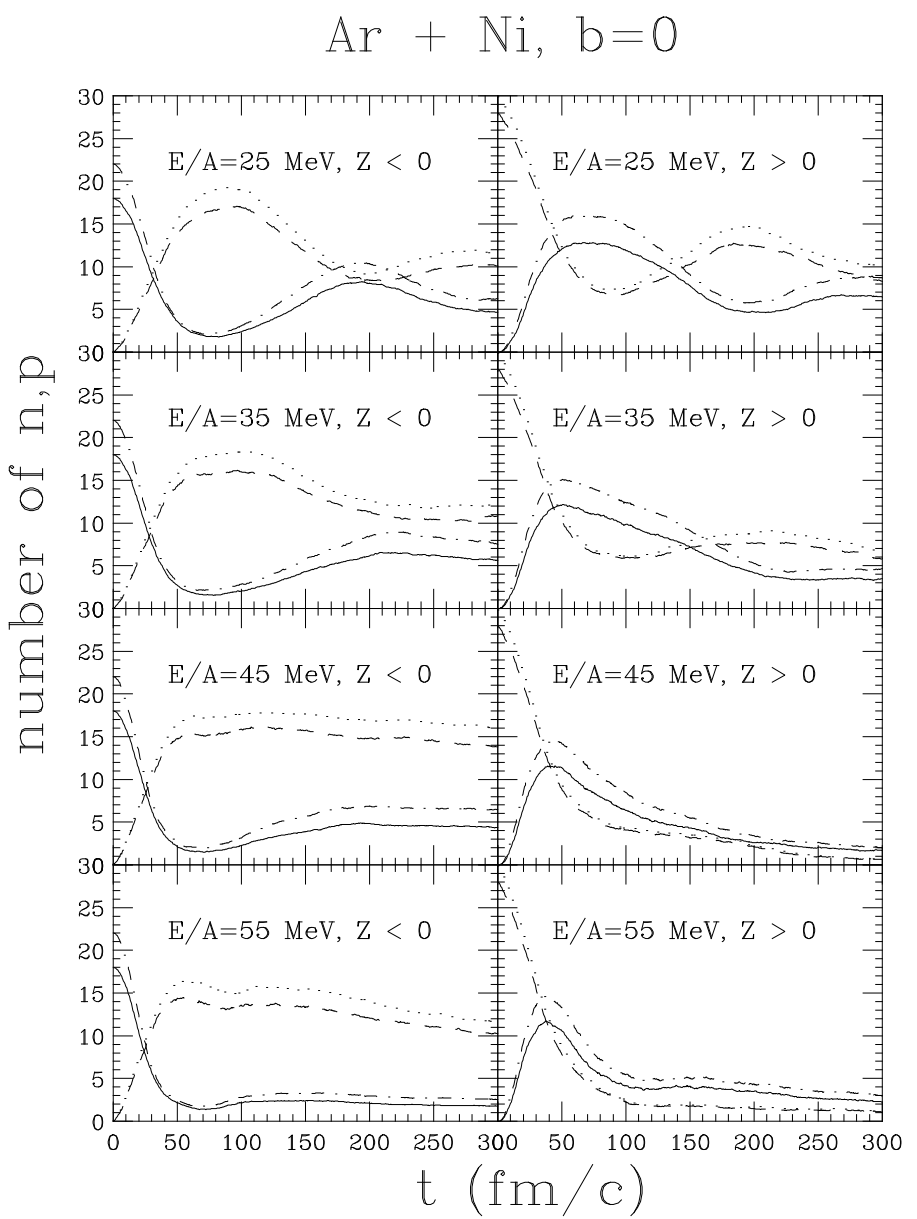

Figure 4.18: The neutron and proton numbers in the residues on the left $(Z<0)$ and right $(Z \geq 0)$ side of the origin. Solid lines are the proton number from the projectile, while dot-dashed lines are the neutron number from the projectile which moves towards the right. Dashed lines are the proton number from the target, while dotted lines are the neutron number from the target which moves towards the left. Taken from Ref. 129 .

Studies on similar systems at much lower energies show that the isospin degree of freedom is one of the fastest to equilibrate [156]. To see if the heavy residues observed above is also in isospin equilibrium, we show in Fig. 4.18 the neutron and proton numbers in the residues on the left $(Z<0)$ and right $(Z \geq 0)$ side of the origin in head-on reactions of $\mathrm{Ar}+\mathrm{Ni}$ at $E_{\text {beam }} / A=25,35,45$ and $55 \mathrm{MeV}$. The solid and dot-dashed lines are, respectively, the proton and neutron numbers from 
the projectile, which is incident from the left. The dashed and dotted lines are, respectively, the proton and neutron numbers from the target, which moves from the right. It is seen that the neutron and proton numbers on both sides not only decrease but also fluctuate. The decreases is mainly due to nucleon-nucleon collisions and particle emissions, while the fluctuation is due to both the restoring force from the mean-field potential and nucleon-nucleon collisions. At $E_{\text {beam }} / A=25 \mathrm{MeV}$ the neutron and proton numbers on the two sides become very close to each other and the amplitude of oscillation is rather small by the time of $300 \mathrm{fm} / \mathrm{c}$. This indicates that the heavy residue is very close to isospin equilibrium, i.e., the proton and neutron distributions are independent of space-time. The damping of the oscillation is faster at $E_{\text {beam }} / A=35 \mathrm{MeV}$ so the particle distribution reaches isospin equilibrium also sooner. The isotopic composition of fragments emitted from the residues in these low energy reactions after about $300 \mathrm{fm} / \mathrm{c}$ would therefore essentially reflect the $(N / Z)_{\mathrm{cs}}$ ratio of the initial composite system, and there is little forward-backward asymmetry. These features are in good agreement with those found in the data of $E_{\text {beam }} / A=25$ and $35 \mathrm{MeV}$ 152, 153, 154].

At higher energies, such as $E_{\text {beam }} / A=45$ and $55 \mathrm{MeV}$, there is little oscillation in the overlapping region between the target and projectile. This is mainly because the incoming momenta of projectile-nucleons and target-nucleons are very large so the mean-field potential cannot reverse the directions of motion of many nucleons during a relatively short reaction time. As a result, there exists a large isospin asymmetry or non-equilibration at these two energies. In particular, on the left side of the origin the $\mathrm{N} / \mathrm{Z}$ ratio of the residue is more affected by that of the target while on the right side it is more affected by that of the projectile. However, the $\mathrm{N} / \mathrm{Z}$ ratios on both sides are not simply those of the target and projectile but a combination of the two depending on the complicated reaction dynamics. In the case of $E_{\text {beam }} / A=55 \mathrm{MeV}$, at the time of about $200 \mathrm{fm} / \mathrm{c}$ the heavy residue has broken up into two pieces with some longitudinal collectivity. The forward moving residue has an average $\mathrm{N} / \mathrm{Z}$ ratio of about $6 / 5$ and an excitation energy of about $8.6 \mathrm{MeV} /$ nucleon, while the backward moving residue has an average $\mathrm{N} / \mathrm{Z}$ ratio of about 16.5/14 and an excitation energy of about $6.8 \mathrm{MeV} /$ nucleon. Both residues are thus in approximate thermal equilibrium in their own center of mass frame [129 but not in thermal equilibrium with each other.

The signature of nuclear transparency from studying the isotopic ratios is consistent with that from studying the kinematic observables, such as the scaled quadrupole moment $Q_{Z Z} / A_{\text {res }}$ defined as

$$
Q_{z z} / A_{\text {res }}(t)=1 / A_{\text {res }} \int \frac{d \vec{r} d \vec{p}}{(2 \pi)^{3}}\left(2 p_{z}^{2}-p_{x}^{2}-p_{y}^{2}\right) f(\vec{r}, \vec{p}, t),
$$

where $f(\vec{r}, \vec{p}, t)$ is the Wigner function from the BUU model calculations and $A_{\text {res }}$ is the mass of the residue. A zero value of $Q_{z z}$ is a necessary but not a sufficient condition for thermal equilibrium, and a positive value of $Q_{Z Z}$ signals transparency. The quadrupole moments of heavy residues formed in the reactions at $E_{\text {beam }} / A=25$ and $35 \mathrm{MeV}$ are about zero by the time of about $300 \mathrm{fm} / \mathrm{c}$, indicating the establishment of thermal equilibrium. Since the heavy residue formed in the reaction at $E_{\text {beam }} / A=$ 
$55 \mathrm{MeV}$ has already broken up into two pieces at about $200 \mathrm{fm} / \mathrm{c}$, it is interesting to examine more closely the evolution of $Q_{z z} / A_{\text {res }}$ in this reaction, which is shown in Fig. 4.19 for particles at local densities larger than $\rho_{0} / 8$ for the reactions at $\mathrm{E} / \mathrm{A}=45$ and $55 \mathrm{MeV}$. It is seen that $Q_{z z}$ has a small positive value when the system breaks up,

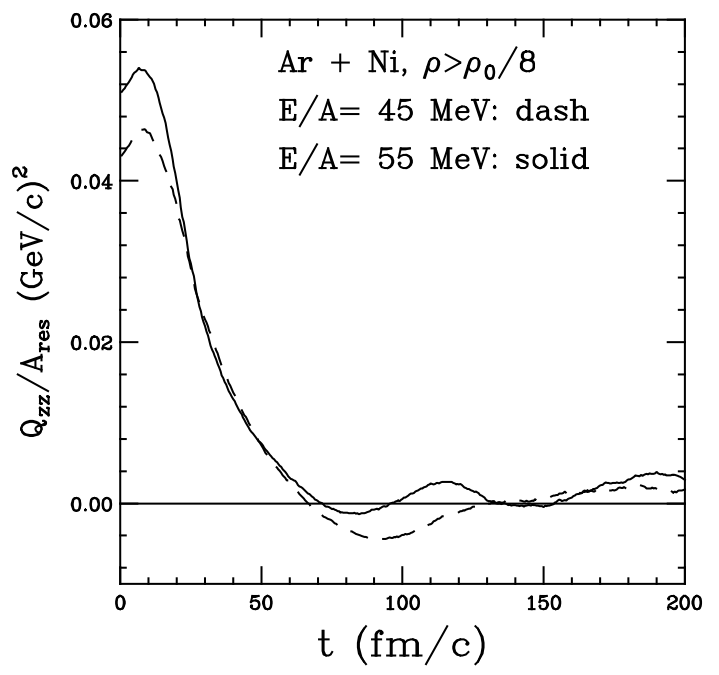

Figure 4.19: The scaled quadrupole moment of heavy residues formed in head-on collisions of $\mathrm{Ar}+\mathrm{Ni}$ at $E_{\text {beam }} / A=45$ and $55 \mathrm{MeV}$. Taken from Ref. [129].

indicating thus a transparency in the collisions. This finding is also in good agreement with the kinematic signature of transparency found recently at Ganil for the same reaction system at similar beam energies using the INDRA detector [157. We note that in recent calculations using the Antisymmetrized Molecular Dynamics (AMD) transparency was found at $35 \mathrm{MeV} /$ nucleon for head-on collisions of ${ }^{40} \mathrm{Ca}+{ }^{40} \mathrm{Ca}$ [158. Moreover, the signatures of transparency from both the isotopic ratios and the quadrupole moments are also in good agreement with earlier BUU predictions on the systematics of nuclear stopping power [159].

Effects of the symmetry potential on isospin equilibrium have been studied at lower beam energies where isospin equilibrium is usually reached. Within the LV model using the simple symmetry potential energy density of Eq. (2.24) [70], it has been found that a stronger symmetry potential enhances the degree of isospin equilibrium. Shown in Table 4.1 are the values of $N-Z$ in the quasitarget (QT) and quasiprojectile (QP) formed in the reaction of ${ }^{56} \mathrm{Ca}+{ }^{40} \mathrm{Ca}$ at an impact parameter of $7 \mathrm{fm}$ and a beam energy of $15 \mathrm{MeV} /$ nucleon. For comparisons, results from reactions of a symmetric system ${ }^{40} \mathrm{Ca}+{ }^{48} \mathrm{Ca}$ are also listed in the table. In both reactions the total mass and charge numbers are the same. The difference in the values of $N-Z$ for the symmetric system is completely due to the numerical fluctuations of the calculations. In the asymmetric reaction the initial value of $N-Z$ is 16 and 0 for the projectile 
Table 4.1: Values of N-Z for the quasitarget(QT) and quasiprojectile (QP)

\begin{tabular}{|c|c|c|c|}
\hline Reaction/N-Z & $\mathrm{c}=0$ & $\mathrm{c}=20$ & $c=28$ \\
\hline \multirow[t]{2}{*}{${ }^{56} \mathrm{Ca}+{ }^{40} \mathrm{Ca}$} & 3.74 & 4.64 & 5.02 \\
\hline & 8.76 & 5.82 & 4.92 \\
\hline \multirow[t]{2}{*}{${ }^{48} \mathrm{Ca}+{ }^{48} \mathrm{Ca}$} & 6.52 & 5.78 & 5.52 \\
\hline & 6.96 & 5.84 & 5.12 \\
\hline
\end{tabular}

and target, respectively. It is seen that significant stopping is achieved even in the case of no symmetry potential. However, without the symmetry potential isospin equilibrium cannot be reached, and the degree of isospin equilibrium increases with the strength of the symmetry potential.

In summary, isospin equilibration in heavy-ion collisions can be used to study the nature of stopping. The degree of isospin equilibrium depends sensitively on both the in-medium nucleon-nucleon cross section and the EOS of asymmetric nuclear matter. In both experiments and BUU model calculations a transition from isospin equilibrium at lower energies to nonequilibrium at higher energies has been observed. The isospin nonequilibrium signals the onset of nuclear transparency. For head-on collisions of the system $A_{\text {pro }}+A_{\text {tar }} \approx 40+58$ the transparency starts at a beam energy as low as $45 \mathrm{MeV} /$ nucleon.

\subsection{Isospin dependence of nuclear collective flow}

Nuclear collective flow in heavy-ion collisions at intermediate energies has been a subject of intensive theoretical and experimental studies during the last decade; for a general introduction and overview see Ref. [160]. The study of the dependence of collective flow on entrance channel parameters, such as the beam energy, mass number and impact parameter, have revealed much interesting physics about the properties and origin of collective flow. In particular, from studying the beam energy dependence it has been found that the transverse collective flow changes from a negative one to a positive one at an energy $E_{\text {bal }}$, defined as the balance energy, due to the competition between the attractive nuclear mean-field potential and the repulsive nucleon-nucleon collisions 1161, 162, 163, 164, 165, 166, 167, 168, 169. The balance energy has been found to depend sensitively on the mass number, impact parameter and properties of the colliding nuclei, such as the thickness of their surfaces [121]. Furthermore, detailed theoretical studies using transport models (for a review see e.g. [131, 173, 134]) have 
shown that both the strength of transverse flow and the balance energy can be used to extract information about the nuclear equation of state and in-medium nucleonnucleon cross sections [88, 89, 126, 136, 137, 170, 171, 172, 173, 174, 175, 176.

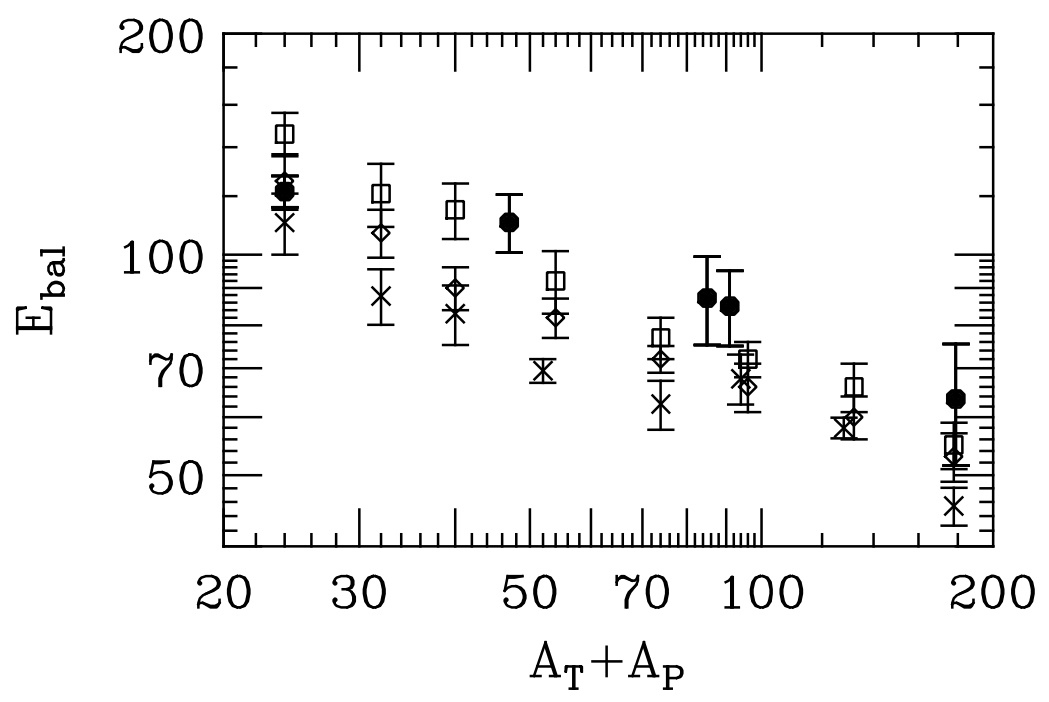

Figure 4.20: The balance energy as a function of the combined mass. The experimental data are compared with BUU calculations using a soft EOS and a reduction of in-medium nucleon-nucleon cross section by $0 \%$ (crosses), 10\% (diamonds) and 20\% (squares) are compared with the data (filled circles). Taken from Ref. [120.

Shown in Fig. 4.20 is the mass dependence of the balance energy measured by Westfall et al. in comparison with the predictions of Klakow et al. using the BUU model [121]. It is seen that BUU calculations reproduce the measured scaling of balance energy with mass, but underpredict the values of balance energy. Using an inmedium cross section of Eq. (3.6) with a parameter of $\alpha=-0.2$ the BUU calculations can better reproduce the data. This results thus indicate that the collective flow and balance energy depend strongly on the in-medium nucleon-nucleon cross section.

Nuclear transverse flow is expected to be isospin-dependent for several reasons. First, it is well-known that nucleon-nucleon collisions cause repulsive collective flow, and this effect is roughly proportional to the number of collisions in the overlapping region. While the number of particles in this region might not be so strongly isospindependent, the number of collisions during the reaction of two neutron-rich nuclei is smaller. This is due to both the smaller neutron-neutron cross sections (see Chapter 3) and the larger Pauli blocking of neutron-neutron collisions in these reactions. This effect is stronger in peripheral collisions of neutron-rich nuclei where two thick neutron skins overlap during the reaction. Second, the Coulomb potential also causes repulsive scatterings, and this effect is obviously weaker in the neutron-richer system. Third, the isospin-independent part of the nuclear mean-field potential is attractive at 
densities up to about $2 \rho_{0}$ and $4 \rho_{0}$, respectively, for the stiff $(K=380 \mathrm{MeV})$ and soft $(K=200 \mathrm{MeV})$ EOS. Since the mean field effect is approximately proportional to the total surface area of the colliding system [121], collisions of neutron-rich nuclei are expected to be influenced by stronger attractive mean-field potentials due to their more extended surfaces. Finally, the generally repulsive symmetry potential is stronger for collisions of neutron-rich nuclei. However, since the magnitude of the symmetry potential is smaller than the isospin-independent mean-field potential, effects of the symmetry potential on the collective flow are expected to be small. Although a more quantitative study on the relative importance of the above mechanisms remains to be worked out, one expects that the overall isospin effect is that the neutron-richer system will have a stronger negative flow below the balance energy and a weaker positive flow at higher energies. Moreover, the relative importance of different contributions to the collective flow depends on the beam energy. As the beam energy increases the repulsive nucleon-nucleon collisions become dominant and effects of the neutron skin is thus less important. Also, the isospin dependence of the nucleon-nucleon cross sections becomes weaker at higher energies as discussed in Chapter 3. It is therefore understandable that the isospin effects on the collective flow decrease as the beam energy increases. These discussions thus show that both the strength of flow and the balance energy are expected to be isospin-dependent.

The isospin dependence of collective flow and balance energy has recently been studied theoretically with isospin-dependent BUU models [130, 177] and experimentally by Pak et al. at NSCL/MSU [178, 179. Within the isospin-dependent BUU model the standard transverse momentum analysis [170] (see also [160]) are carried out for two reaction systems, ${ }^{48} \mathrm{Cr}+{ }^{58} \mathrm{Ni}$ and ${ }^{48} \mathrm{Ca}+{ }^{58} \mathrm{Fe}$, which have the same mass number of $48+58$ but different neutron/proton ratios of 1.04 and 1.30 , respectively. Typical results for central collisions at an impact parameter of $2 \mathrm{fm}$ and beam energies of 50 , 60 and $70 \mathrm{MeV} /$ nucleon are shown in Fig. 4.21. At a beam energy of $50 \mathrm{MeV} /$ nucleon, the transverse flow in the reaction of ${ }^{48} \mathrm{Ca}+{ }^{58} \mathrm{Fe}$ is still negative while that in the reaction of ${ }^{48} \mathrm{Cr}+{ }^{58} \mathrm{Ni}$ becomes already positive. The difference disappears at beam energies above $70 \mathrm{MeV} /$ nucleon.

More quantitatively, one can compare the flow parameter $F$ defined as the slope of the transverse momentum distribution at the center of mass rapidity $y_{\mathrm{cm}}$. The beam energy dependence of the flow parameter for the two reaction systems at impact parameters of 2 and $5 \mathrm{fm}$ are shown in Fig. 4.22. The lines are the least-square fit to calculations using linear functions, i.e., $F(\mathrm{Ca}+\mathrm{Fe})=-32.2+0.55 E / A$ and $F(\mathrm{Cr}+\mathrm{Ni})=-23.9+0.48 E / A$ at $b=2 \mathrm{fm}$; and $F(\mathrm{Ca}+\mathrm{Fe})=-35.9+0.22 E / A$ and $F(\mathrm{Cr}+\mathrm{Ni})=-23.2+0.18 E / A$ at $b=5 \mathrm{fm}$. It is seen that in both central and peripheral collisions the neutron-rich system ${ }^{48} \mathrm{Ca}+{ }^{58} \mathrm{Fe}$ shows systematically smaller flow parameters, indicating thus a stronger attractive interaction during the reaction. The effect is more appreciable in peripheral collisions - as expected. Consequently, the balance energy in ${ }^{48} \mathrm{Ca}+{ }^{58} \mathrm{Fe}$ reaction is higher than that in the reaction of ${ }^{48} \mathrm{Cr}+{ }^{58} \mathrm{Ni}$ by about 10 to $30 \mathrm{MeV} /$ nucleon. The difference between flow parameters in the two systems decreases as the beam energy increases and finally disappears as the beam energy rises far above the balance energy.

The experimental investigation of the isospin dependence of transverse collective 


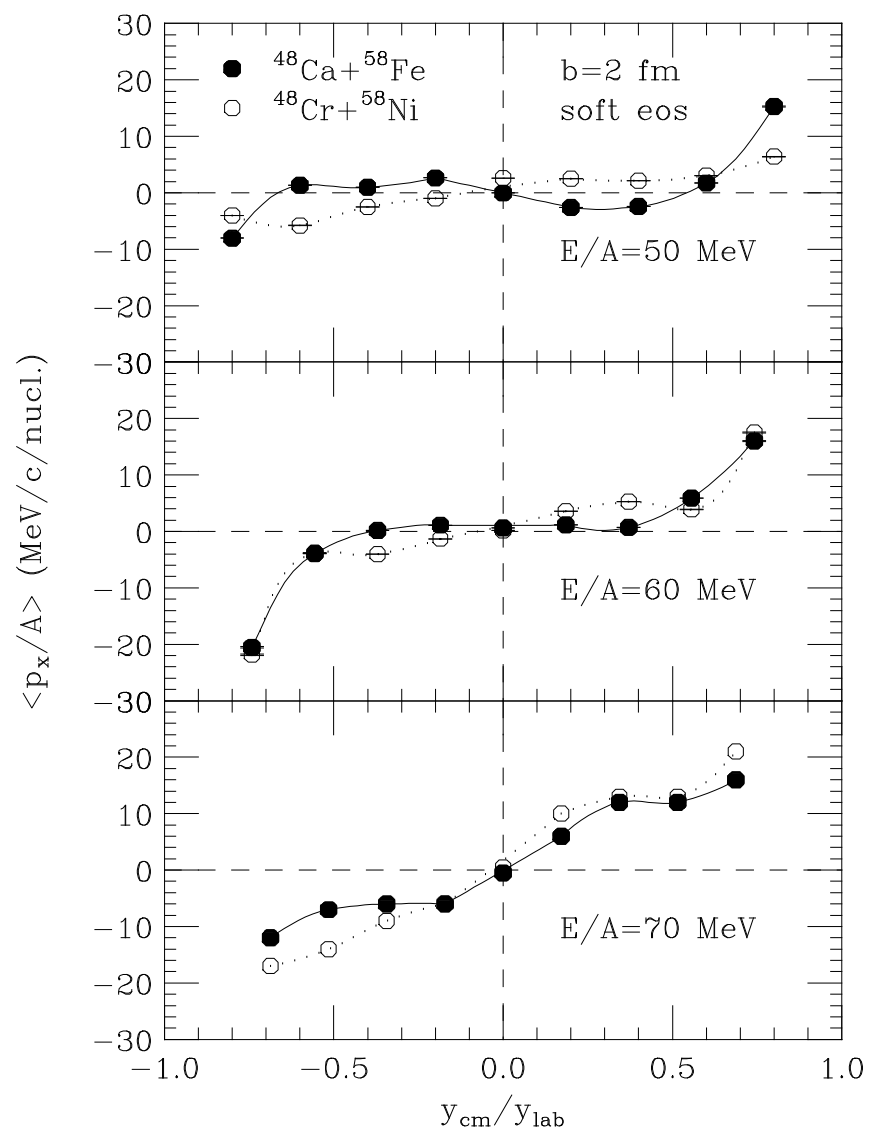

Figure 4.21: The transverse momentum distribution in the reactions of ${ }^{48} \mathrm{Cr}+{ }^{58} \mathrm{Ni}$ and ${ }^{48} \mathrm{Ca}+{ }^{58} \mathrm{Fe}$ at an impact parameter of $2 \mathrm{fm}$ and beam energies of 50, 60 and 70 $\mathrm{MeV} /$ nucleon, respectively. Taken from Ref. [130]. 


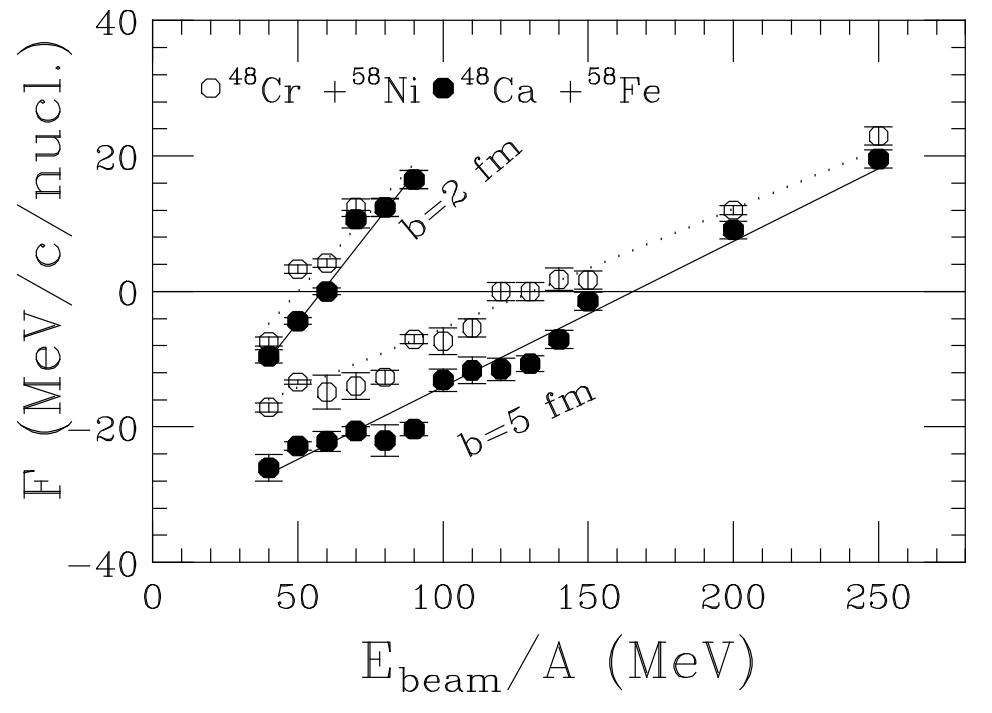

Figure 4.22: Flow parameters for the reactions of ${ }^{48} \mathrm{Cr}+{ }^{58} \mathrm{Ni}$ and ${ }^{48} \mathrm{Ca}+{ }^{58} \mathrm{Fe}$ at impact parameters of 2 and $5 \mathrm{fm}$. Taken from Ref. [130].

flow has been recently carried out by Pak et al. et al. at NSCL/MSU. Since one cannot identify the direction of flow using the transverse momentum analysis in experiments, the absolute value of flow parameter is usually extracted. Shown in Fig. 4.23 are the flow parameters of particles with charge $Z=1, Z=2$ and $Z=3$ as functions of reduced impact parameter $b / b_{\max }$ for the reactions of ${ }^{58} \mathrm{Fe}+{ }^{58} \mathrm{Fe}$ and ${ }^{58} \mathrm{Ni}+{ }^{58} \mathrm{Ni}$ at a beam energy of $55 \mathrm{MeV} /$ nucleon. Note that at this beam energy flow is still dominated by the attractive mean-field potential and is thus negative if the same convention as in the BUU calculations is used. It is seen that the flow parameter for the neutron-richer system is consistently higher and is in agreement with the BUU predictions. Pak et al. have also studied the flow parameter as a function of the isotope ratio of the composite projectile plus target system for three different fragment types from three isotopic entrance channels. Shown in the upper window of Fig. 4.24 is the mean transverse momentum in the reaction plane versus the reduced c.m. rapidity for $\mathrm{Z}=2$ fragments from impact-parameter-inclusive ${ }^{58} \mathrm{Mn}+{ }^{58} \mathrm{Fe}$ collisions at $55 \mathrm{MeV} /$ nucleon. The flow parameter extracted for the impact-parameter-inclusive events is plotted in the lower window of Fig. 4.24 as a function of the ratio of neutrons to protons of the combined system $(N / Z)_{\mathrm{cs}}$. The flow parameter increases linearly with the ratio $(N / Z)_{\mathrm{cs}}$ for all three types of particles. Fig. 4.25 shows the measured energy dependence of the flow parameter for midcentral collisions. The curves are drawn to guide the eyes. In the whole energy range the flow parameter shows dependence on the isospin asymmetry of the colliding 


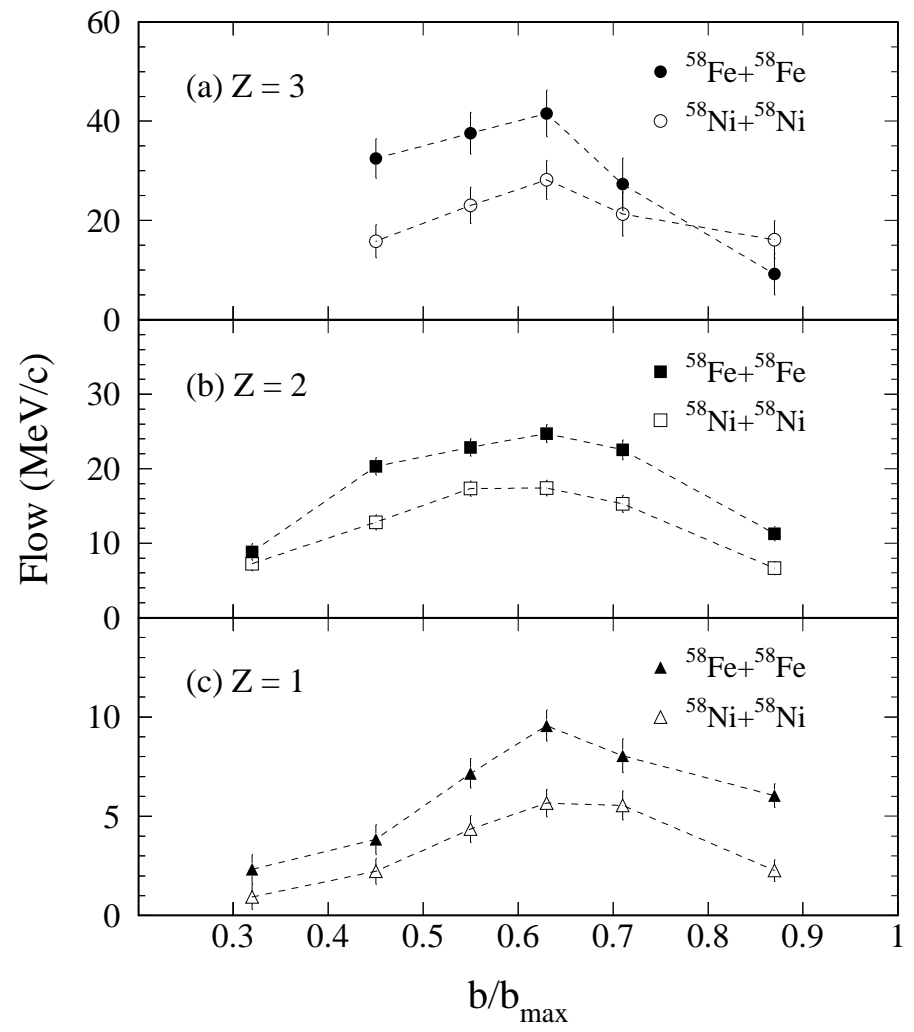

Figure 4.23: Flow parameters for the reactions of ${ }^{58} \mathrm{Fe}+{ }^{58} \mathrm{Fe}$ and ${ }^{58} \mathrm{Ni}+{ }^{58} \mathrm{Ni}$ as a function of the reduced impact parameter at a beam energy of $55 \mathrm{MeV} /$ nucleon. Taken from Ref. 178. 

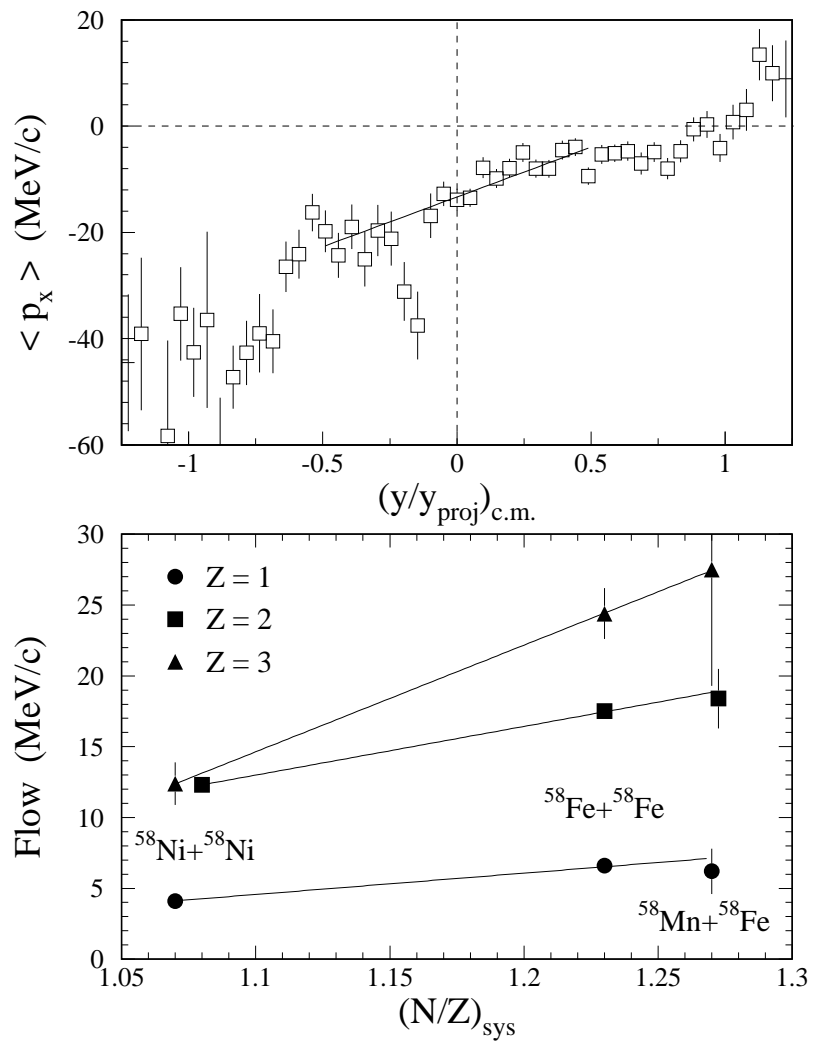

Figure 4.24: (Upper window) Mean transverse momentum in the reaction plane versus the reduced c.m. rapidity for $\mathrm{Z}=2$ fragments from impact-parameter-inclusive ${ }^{58}+{ }^{58}$ $\mathrm{Fe}$ collisions at $55 \mathrm{MeV} /$ nucleon. (Lower window) Isospin dependence of the flow parameter for inclusive collisions at a beam energy of $55 \mathrm{MeV} /$ nucleon. Taken from Ref. 178. 


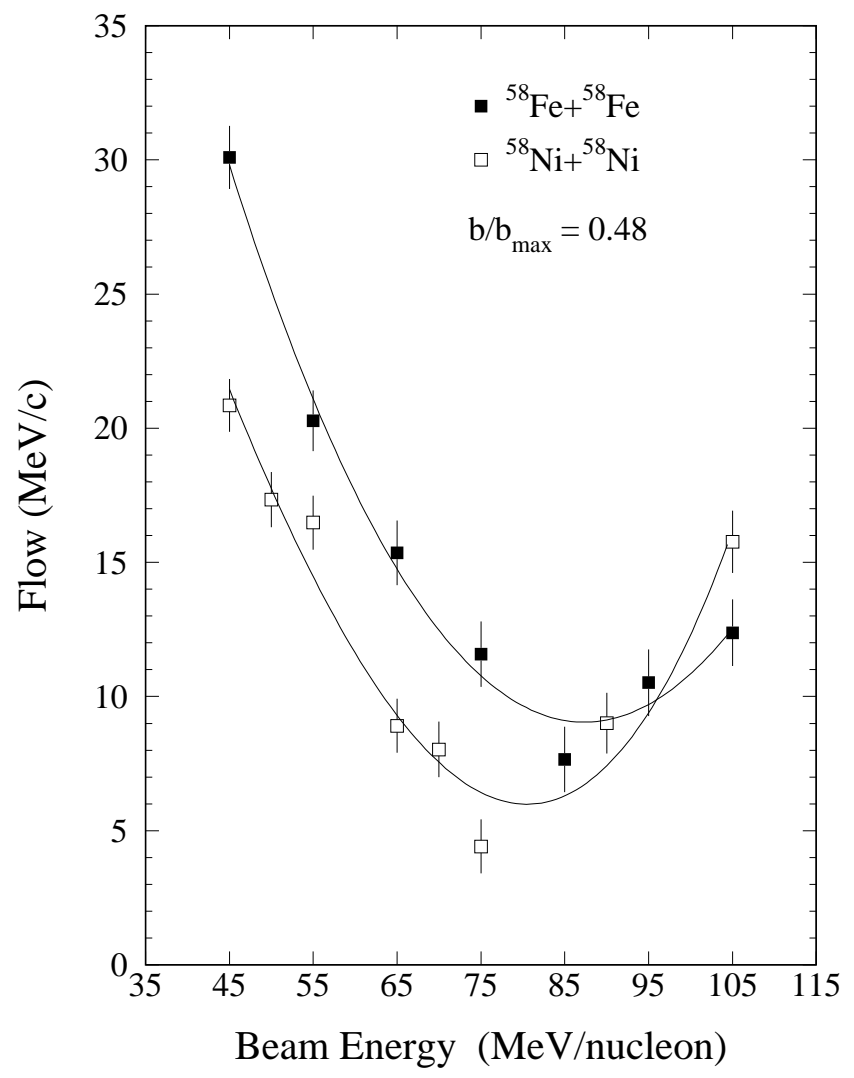

Figure 4.25: Energy dependence of the flow parameter for midcentral collisions of ${ }^{58} \mathrm{Fe}+{ }^{58} \mathrm{Fe}$ and ${ }^{58} \mathrm{Ni}+{ }^{58} \mathrm{Ni}$, respectively. Taken from Ref. [179]. 
system. The minima of the curves correspond to the balance energies where the flow changes sign. Because of the isospin dependence of nucleon-nucleon cross sections, there are less number of nucleon-nucleon collisions in the neutron-rich system. Below the balance energy attractive mean-field potentials dominate. There fewer collisions mean less kinetic pressure and help generate more attractive flow in the neutron-rich system. Above the balance energy, where the repulsive nucleon-nucleon collisions dominate, less collisions in the neutron-rich system results in a smaller flow parameter. For both systems studied in the experiment the balance energy occurs at about $E_{\text {beam }} / A=90 \mathrm{MeV}$. These features in the measured energy dependence are also in quantitative agreement with those predicted in Fig. 4.22 using the BUU model.

The comparison of theoretical predictions on collective flow with the experimental data is shown in Fig. 4.26. In the upper window the measured and predicted balance energies are compared for both systems, and in the lower window the measured and predicted differences in balance energies of the two systems are compared. One observes that the difference of balance energies, i.e., the $\delta E_{\mathrm{bal}}$, is well reproduced. The model, however, underpredicts the balance energy in central collisions and overpredicts the data in peripheral collisions. These discrepancies can be understood as follows [120, 122, 180]. We mentioned earlier in this section that in central collisions nucleon-nucleon collisions are important, and a reduction of nucleon-nucleon cross sections by about $20 \%$ was needed to reproduce the measured balance energy - see Fig. 4.20. Once one includes the isospin dependence, we expect a different reduction of the in-medium nucleon-nucleon cross sections is needed to reproduce the data. This remains to be studied. In the case of peripheral collisions, the mean-field potential has a more important effect on flow than nucleon-nucleon collisions. In particular, the repulsive momentum-dependent mean-field potential [88, 90, 92, 93, which is not included in the calculations presented here, is expected to reduce the balance energy in heavy ion collisions at large impact parameters [180].

It is encouraging that both model calculations and experiments show that the transverse flow is sensitive to the isospin asymmetry of the colliding systems. Studies using the isospin-dependent BUU model indicate, however, that effects of the symmetry potential on the transverse flow and balance energy are not appreciable, because they are small compared to those of the isospin-independent part of the nuclear EOS. By neglecting the Coulomb interaction, the balance energies for both systems increase, but the difference remains about the same in central collisions and becomes larger in peripheral collisions. Thus, the observed isospin dependence of the transverse flow and balance energy is mainly due to the isospin-dependent in-medium nucleonnucleon cross sections. The isospin-dependent BUU model reproduces the measured isospin dependence of the balance energy, but fails to reproduce the magnitude of the balance energy. It thus indicates the need of a reduced in-medium nucleon-nucleon cross section and a momentum-dependent mean-field potential. More detailed work in refining the comparison between model calculations and the data remains to be done to infer useful information about the in-medium nucleon-nucleon cross sections. 


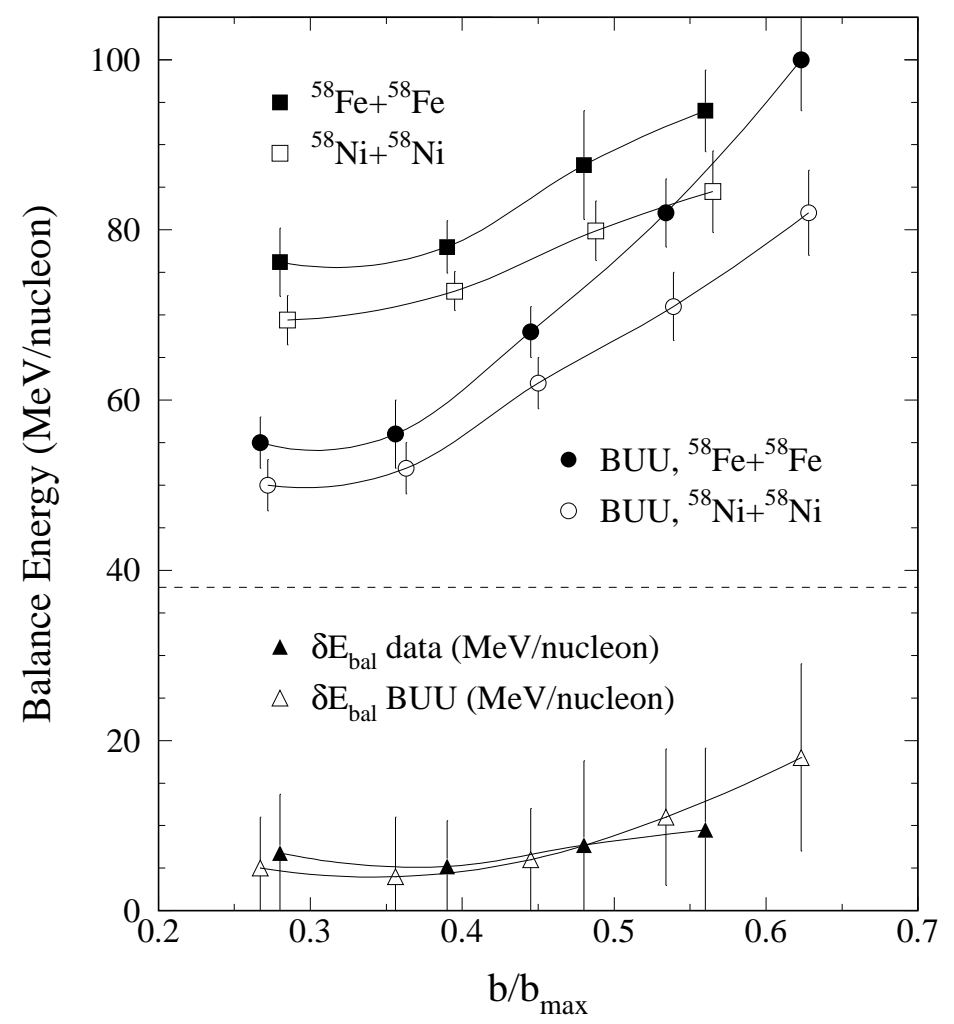

Figure 4.26: Upper window: comparison of the measured and calculated balance energies for collisions of ${ }^{58} \mathrm{Fe}+{ }^{58} \mathrm{Fe}$ and ${ }^{58} \mathrm{Ni}+{ }^{58} \mathrm{Ni}$ as functions of impact parameter. Lower window: comparison of the difference in balance energies of the two systems as a function of impact parameter. Taken from Ref. [179]. 


\subsection{Isospin dependence of total reaction cross sec- tions and radii of neutron-rich nuclei}

We discuss, in this section, the isospin dependence of total reaction cross sections of neutron-rich nuclei. The total reaction cross section provides direct information about the size of nuclei involved in the reaction, and has been one of the standard techniques for studying the size and structure of radioactive nuclei [5]. Theoretical analyses of the measured reaction cross sections have been done almost exclusively by using the Glauber model or eikonal approximation with various modifications. Shown in Fig. 4.27 is the total reaction cross section for ${ }^{12} \mathrm{C}+{ }^{12} \mathrm{C}$ reactions at beam energies from about 10 to $2000 \mathrm{MeV} /$ nucleon [181]. The model works surprisingly well in the whole energy range, although it should not be an appropriate model at low energies. Indeed, recent Glauber model calculations underpredict significantly the interaction cross sections for ${ }^{11} \mathrm{Li}+\mathrm{C}$ reactions at beam energies below about 100 $\mathrm{MeV} /$ nucleon [182, 183]. Furthermore, one expects that both the nuclear EOS and the

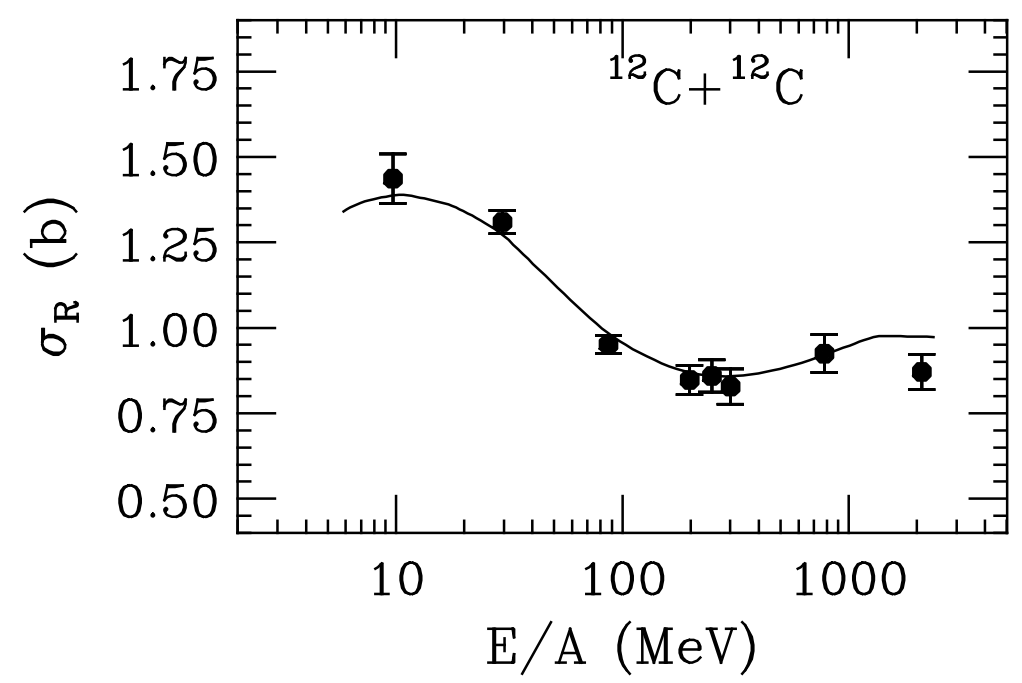

Figure 4.27: The energy dependence of the total reaction cross section for the reaction of ${ }^{12} \mathrm{C}+{ }^{12} \mathrm{C}$. Taken from Ref. [181].

in-medium nucleon-nucleon cross sections, which one has learned from intermediate energy heavy-ion collisions, should have effects on the total reaction cross section. In the following, we shall first review the observed isospin dependence of total reaction cross sections, and then comment on the feasibility of applying isospin-dependent transport models to study the total reaction cross section. As we shall show that results from these studies have already demonstrated the possibility of learning the nuclear EOS, in-medium nucleon-nucleon cross section, and the size of nuclei from the 
total reaction cross sections.

Several empirical formulae for describing the systematics of the total reaction cross sections have been developed based on the Glauber model [181, 185, 184]. For example, Kox's parameterization for the total reaction cross section can be written as 181

$$
\sigma_{R}(E)=\pi r_{0}^{2} f(E)
$$

where $f(E)$ is an empirical expression describing the degree of transparency, and depends on the masses of the colliding nuclei and the beam energy. The strongabsorption radius parameter $r_{0}$ contains information about the size of both the target and projectile. A constant value of $r_{0} \approx 1.05 \mathrm{fm}$ independent of the beam energy and projectile-target combination has been obtained by analyzing a large amount of $\sigma_{R}(E)$ data for collisions between $\beta$-stable nuclei at energies from 33 to 2100 $\mathrm{MeV} /$ nucleon. Using the same parameterization for reactions involving neutron-rich nuclei $r_{0}^{2}$ has been found to increase with increasing neutron excess in the reaction system [5, 184, 186]. Shown in Fig. 4.28 are the extracted values of $r_{0}^{2}$ as a function

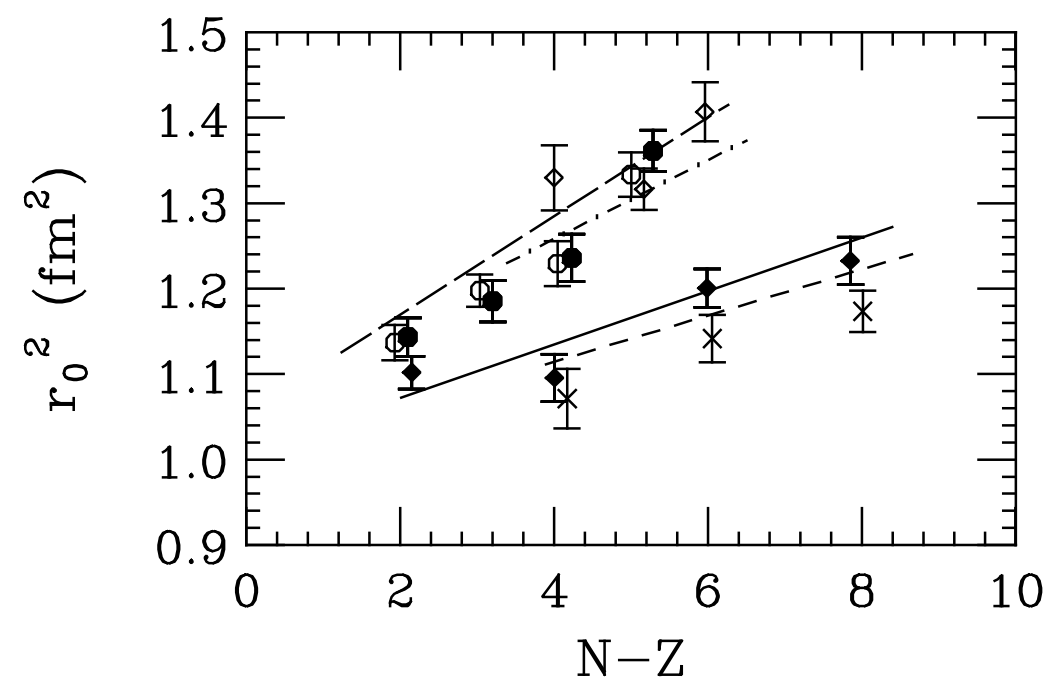

Figure 4.28: Square of the radius parameter of the interacting nuclei $r_{0}^{2}$ as a function of $N-Z$ of the reaction system. The open circles, filled diamonds, open diamonds, crosses and filled circles are for ${ }^{16-18} \mathrm{~N}+{ }^{28} \mathrm{Si},{ }^{26-28} \mathrm{Na}+{ }^{28} \mathrm{Si},{ }^{12} \mathrm{C}+{ }^{62-68} \mathrm{Zn}$ and ${ }^{18-21} \mathrm{O}+{ }^{28} \mathrm{Si}$, respectively. Taken from Ref. [184].

of the neutron excess $N-Z$ of the reaction system [186]. The linear dependence of $r_{0}^{2}$ on $N-Z$ can be explained using an extended Glauber model where nucleons are assumed to have a Fermi-type distribution with the following isospin-dependent surface diffuseness for neutrons [184]

$$
t_{n}=2.4+10 \cdot\left(\delta-\delta_{\beta}\right)(\mathrm{fm}),
$$


where $\delta_{\beta} \equiv((N-Z) / A)_{\beta}$ is the relative neutron excess along the $\beta$ stability line.

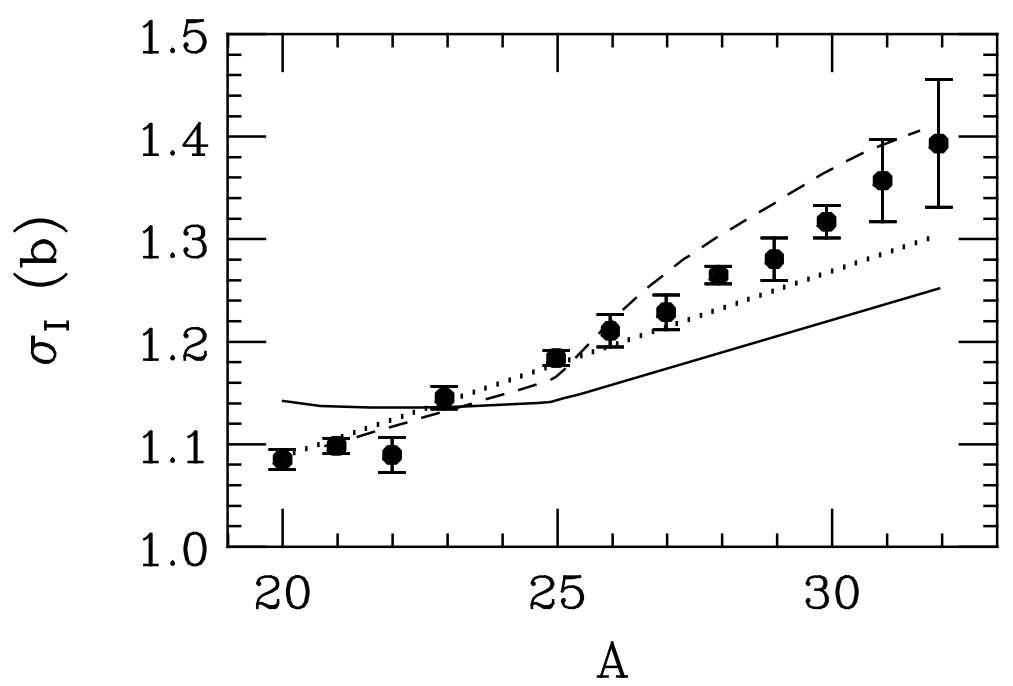

Figure 4.29: Interaction cross section for $\mathrm{Na}$ isotopes on carbon at a beam energy of $950 \mathrm{MeV} /$ nucleon. Taken from Ref. [187.

More recently, Suzuki et al. have measured the interaction cross section of $\mathrm{Na}$ isotopes $\left({ }^{20-23,25-32} \mathrm{Na}\right)$ on a carbon target at a beam energy of $950 \mathrm{MeV} /$ nucleon [187. In Fig. 4.29 the measured cross section (filled circles) is plotted as a function of mass number of the $\mathrm{Na}$ isotopes. The dotted line is obtained from the expression

$$
\sigma_{I}=\pi\left(R_{I}(C)+r_{0} A^{1 / 3}\right)^{2}
$$

where $R_{I}(C)=2.61 \mathrm{fm}$ is the interaction radius of ${ }^{12} \mathrm{C}$ and $r_{0}$ is chosen to reproduce the ${ }^{23} \mathrm{Na}+{ }^{12} \mathrm{C}$ data. It is seen that as the projectile becomes more neutron-rich this simple scaling starts to deviate from the data. This result is consistent with that observed in Refs. [184, 186], although different formulae have been used there to describe the reaction cross section. The results from the Glauber model are also shown in the figure. The solid line is obtained by assuming that the density distribution for neutrons is the same as that for protons, except a normalization factor $(\mathrm{N} / \mathrm{Z})$. With this assumption the model underpredicts the cross section for neutron-rich nuclei but overpredicts that for the neutron-poor nuclei. Using the neutron and proton density distributions predicted by the RMF theory, the data can be well reproduced as shown by the dashed line. The increasing difference between the dashed and solid lines reflects the effects due to the larger neutron-skin of $\mathrm{Na}$ isotopes. In fact, a gradual increase of the neutron-skin up to $0.4 \mathrm{fm}$ for neutron-rich $\mathrm{Na}$ isotopes has been deduced from fitting the total reaction cross sections using the Glauber model 187. 
In the optical limit, the Glauber model predicts a reaction cross section

$$
\sigma_{R}=2 \pi \int_{0}^{\infty}[1-T(b)] b d b,
$$

where $T(b)$ is the transmission function at an impact parameter $b$. In the standard Glauber model, $T(b)$ is evaluated from a convolution of the nucleon-nucleon cross section $\sigma_{k l}$, with the density distributions of target $(T)$ and projectile $(P)$ nucleons in the overlapping region 185, i.e.,

$$
T(b)=\exp \left(-\sum_{k l} \sigma_{k l} \int \rho_{T k}^{z}(\vec{s}) \rho_{P l}^{z}(\vec{b}-\vec{s}) d \vec{s}\right),
$$

where the indices $k, l$ are used to distinguish neutrons and protons and $\rho^{z}$ is the nucleon density distribution along the beam direction, i.e.,

$$
\rho^{z}(\vec{s})=\int_{-\infty}^{\infty} \rho\left(\sqrt{s^{2}+z^{2}}\right) d z .
$$

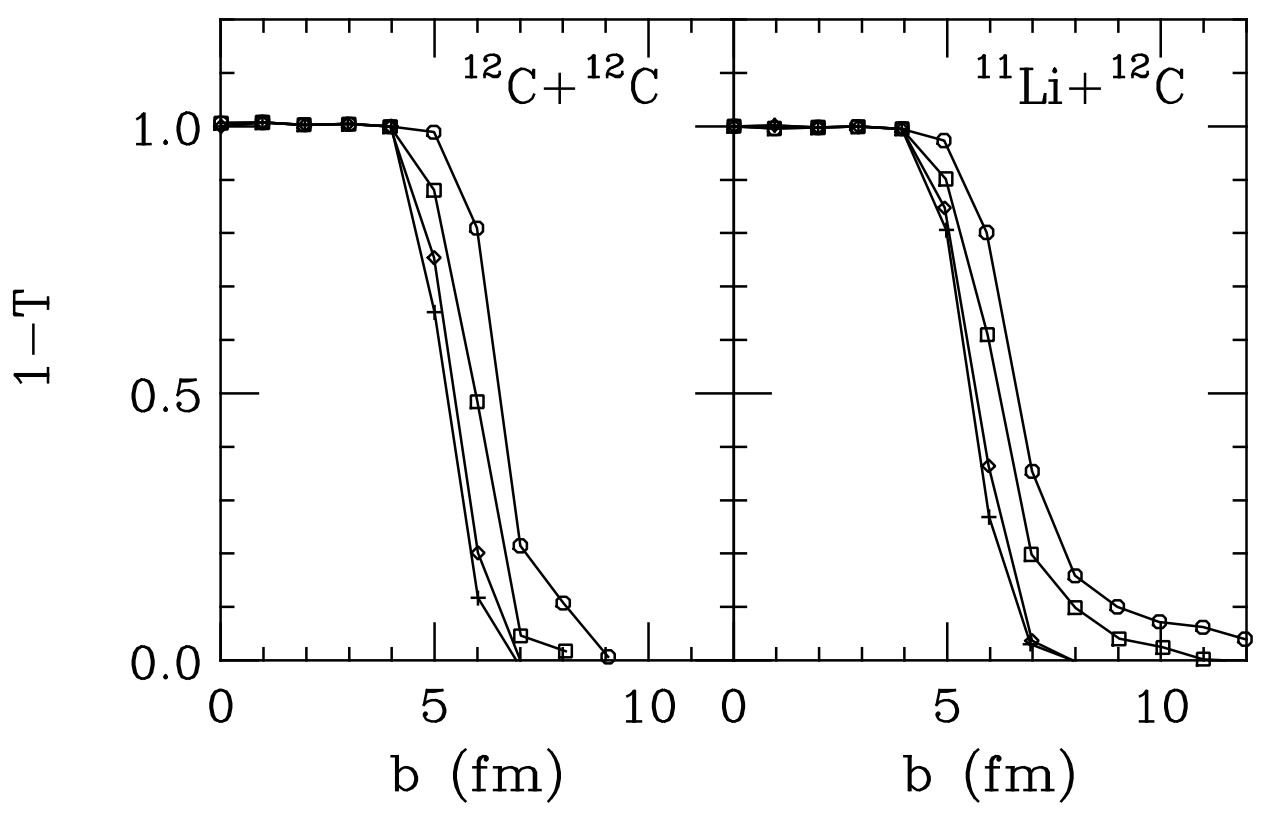

Figure 4.30: The impact parameter dependence of the reaction probability $1-T(b)$ for the reactions of ${ }^{12} \mathrm{C}+{ }^{12} \mathrm{C}$ (left window) and ${ }^{11} \mathrm{Li}+{ }^{12} \mathrm{C}$ (right window) at four beam energies. Circles, squares, diamonds, and crosses represent the results for E/A $=43,75,400$, and $800 \mathrm{MeV}$, respectively. Taken from Ref. [191].

In transport models, the transmission function $T(b)$ can be calculated directly by simply counting events in which no nucleon-nucleon collision have occurred. This approach, however, is hampered by large statistical fluctuations. It is thus numerically more reliable to determine $T(b)$ by using the observation that the number of 
nucleon-nucleon collisions encountered by a nucleon in heavy ion collisions at all impact parameters and for both low and high beam energies can be very well described by a Poisson distribution [159, 188, 189, 190]. Therefore, the transmission function at a given impact parameter $b$ can be simply expressed by the average number of collisions per nucleon $\bar{N}(b)$, i.e.,

$$
T(b)=\exp (-\bar{N}(b)) \text {. }
$$

The isospin dependence of the reaction dynamics is contained in $\bar{N}(b)$. However, the isospin-dependent transport models have not been used to calculate the total reaction cross section. Instead, the normal isospin-independent BUU model was used by Shen et al. in calculating the total cross sections [191]. In their study neutrons and protons are distributed randomly in a sharp sphere with a radius of $r=r_{0} A^{1 / 3}$. By varying the nuclear EOS, in-medium nucleon-nucleon cross section and the radius parameter $r_{0}$ Shen et al. have already demonstrated the usefulness of transport models in studying the total reaction cross section. Fig. 4.30 shows the reaction probability $1-T(b)$ as a function of impact parameter for the reaction of ${ }^{12} \mathrm{C}+{ }^{12} \mathrm{C}$ and ${ }^{11} \mathrm{Li}+{ }^{12} \mathrm{C}$ at the four beam energies from 43 to $800 \mathrm{MeV} /$ nucleon. It is seen that in central collisions these nuclei behave as black spheres. The observed energy dependence of the total reaction cross section is due to the energy dependence of partial transparency in peripheral collisions.

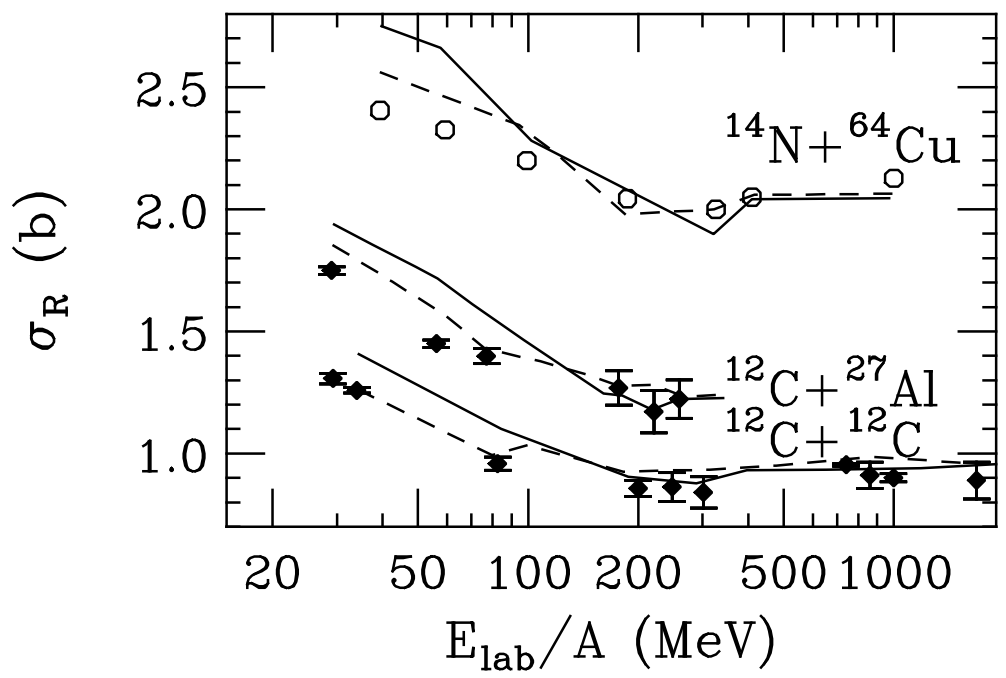

Figure 4.31: The energy dependence of the total reaction cross section for three different reactions. The data are shown by filled circles while the solid (dashed) lines are calculations with the stiff (soft) nuclear EOs. Taken from Ref. [191].

The calculated and measured energy dependence of the total reaction cross section are compared in Fig. 4.31. The filled circles are the experimental data, while the 
solid (dashed) lines are the BUU calculations with a stiff (soft) nuclear EOS. Both calculations can reproduce the data at beam energies above about $100 \mathrm{MeV} /$ nucleon. The dependence of the total cross section to the nuclear Eos appears at lower beam energies, but this is exactly where the Glauber-type models underpredict the total reaction cross sections [182, 183].

BUU calculations generally overpredict the measured cross sections at beam energies below $100 \mathrm{MeV} /$ nucleon. This might be related to the in-medium nucleonnucleon cross sections used in the model. In Ref. [191 a parameterization which fits the proton-proton data and has a cutoff of $55 \mathrm{mb}$ at lower beam energies has been used for all nucleon-nucleon collisions. If the nucleon-nucleon cross section is modified in medium, then the total reaction cross section is expected to be affected as well. This effect is shown in Fig. 4.32, where it is seen that for all incident energies a $20 \%$ variation of the nucleon-nucleon cross section results in about $15 \%$ variation of the total reaction cross section. This variation appears to be larger than that found in Ref. 192 using the Glauber model where a 7\% reduction has been found by reducing the effective, energy-independent nucleon-nucleon cross section $40 \mathrm{mb}$ by $20 \%$. Furthermore, a 20\% reduction the BUU model can better reproduce the data. This seems to be consistent with that from studying the balance energy [120] and the impact parameter dependence of the flow parameter [122]. However, one should note that the $20 \%$ reduction of the proton-proton cross section used in Ref. [191 for all nucleon-nucleon collisions corresponds to a larger reduction of the in-medium nucleon-nucleon cross section if its isospin dependence is taken into account.

Transport models may also be useful for studying the isospin-dependence of the radius parameter $r_{0}$. Keeping the $r_{0}$ parameter fixed at $1.33 \mathrm{fm}$ for ${ }^{12} \mathrm{C}$ the calculated total reaction cross section for ${ }^{11} \mathrm{Li}+{ }^{12} \mathrm{C}$ has been found to be very sensitive to the $r_{0}$ parameter for ${ }^{11} \mathrm{Li}$. Shown in Fig. 4.33 are the $r_{0}$ dependence of the total reaction cross section. As one expects, the total reaction cross section increases significantly with increasing $r_{0}$ for ${ }^{11} \mathrm{Li}$. A radius parameter $r_{0}=1.60 \mathrm{fm}$ is needed to fit the cross section data.

From the above discussion it is clear that transport models are useful for studying the total reaction cross section, especially at low beam energies where Glauber-type models are not valid. So far only the isospin-independent BUU model has been used to study the total reaction cross section and its dependence on the nuclear EOs, inmedium nucleon-nucleon cross section, and the size of neutron-rich nuclei. It will be interesting to repeat such studies using the isospin-dependent transport models.

\subsection{Isospin dependence of subthreshold pion pro- duction}

The multiplicity ratio $\pi^{-} / \pi^{+}$of charged pions in heavy-ion collisions is closely related to the ratio $N / Z$ in the participant region. A systematic study of the relation between the two ratios are useful not only for extracting information about the nucleon distributions but also for studying the production mechanism of subthreshold pions. 


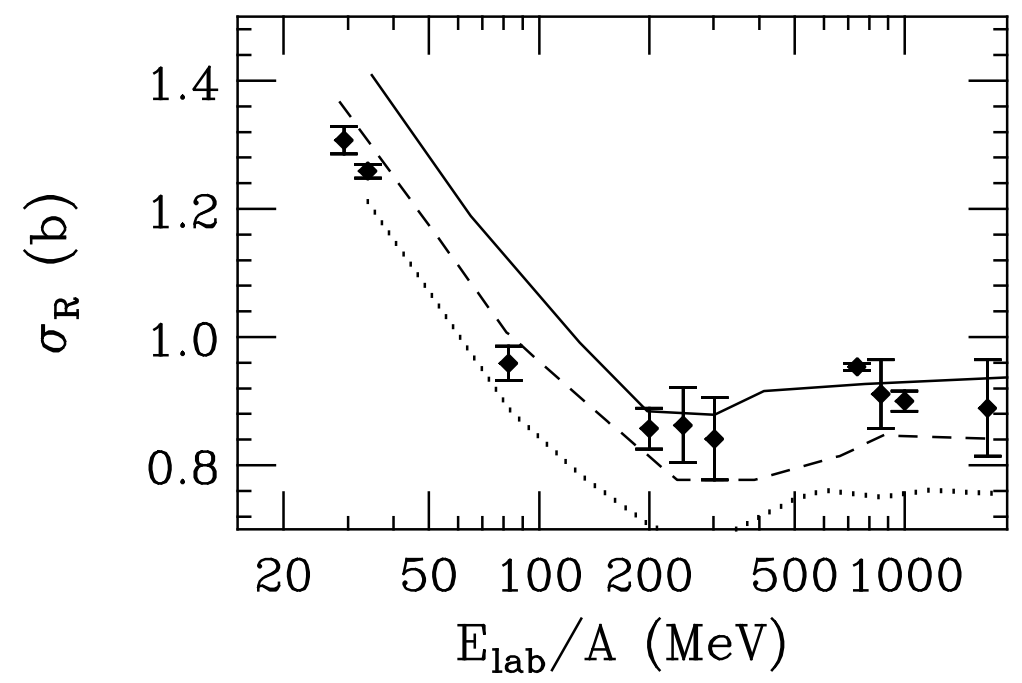

Figure 4.32: Effects of in-medium nucleon-nucleon cross sections on the energy dependence of total reaction cross section for the reaction of ${ }^{12} \mathrm{C}+{ }^{12} \mathrm{C}$. The data are plotted with filled circles. All calculations are carried out with the stiff nuclear EOs. The solid line is obtained using the free-space $p p$ cross section; while the dashed and dotted lines are calculated with a reduction of the cross section by $20 \%$ and $40 \%$, respectively. Taken from Ref. [191]. 


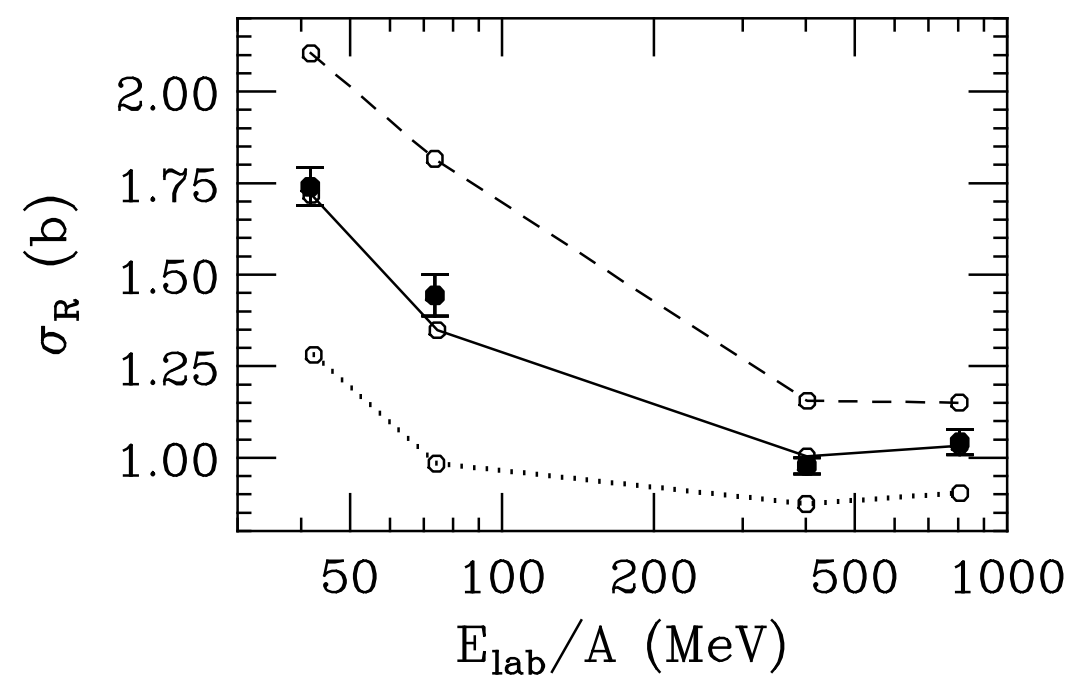

Figure 4.33: Effects of radius parameter $r_{0}$ on the energy dependence of total reaction cross section for the reaction of ${ }^{11} \mathrm{Li}+{ }^{12} \mathrm{C}$. The data are plotted with filled circles. The calculations are represented by the open symbols and connected by the dashed, solid, and dotted lines, representing the choices of $r_{0}=1.8,1.6$, and $1.33 \mathrm{fm}$, respectively. All calculations are carried out with the stiff nuclear EOs. Taken from Ref. [191].

Assuming that pions are produced through the intermediate $\Delta(1232)$ resonance, i.e., $N N \rightarrow N \Delta(1232)$ and $\Delta \rightarrow N+\pi$, the ratio $\pi^{-} / \pi^{+}$can be estimated [193, 194] and is given by

$$
\frac{\pi^{-}}{\pi^{+}}=\frac{5 N^{2}+N Z}{5 Z^{2}+N Z} \approx\left(\frac{N}{Z}\right)^{2}
$$

This simple scaling formula is supported by data from relativistic heavy-ion experiments at the BEVALAC [193, 195]. Fig. 4.34 shows the $\pi^{-} / \pi^{+}$ratio as a function of $N / Z$ ratio of the participants in the reaction of $\mathrm{Ar}+\mathrm{KCl}$ at a beam energies of $2.1 \mathrm{GeV} /$ nucleon. It is seen that the $\pi^{-} / \pi^{+}$ratio scales well with $(N / Z)^{2}$ of the participants. At subthreshold energies, however, it has been found that the ratio $\pi^{-} / \pi^{+}$scales approximately linearly with $N / Z$ from in reactions induced by ${ }^{12} \mathrm{C}$ on ${ }^{7} \mathrm{Li},{ }^{12} \mathrm{C},{ }^{116} \mathrm{Sn}$ and ${ }^{124} \mathrm{Sn}$ at $E_{\text {beam }} / A=60-85 \mathrm{MeV}$ [196, 197]. For example, at $E_{\text {beam }} / A=85 \mathrm{MeV}$ the relative ratio for reactions with ${ }^{124} \mathrm{Sn}$ and ${ }^{116} \mathrm{Sn}$ targets is

$$
\left(\frac{\sigma_{\pi^{-}}}{\sigma_{\pi^{+}}}\right)\left({ }^{124} \mathrm{Sn}\right) /\left(\frac{\sigma_{\pi^{-}}}{\sigma_{\pi^{+}}}\right)\left({ }^{116} \mathrm{Sn}\right) \approx 1.2 \pm 0.3
$$

which is compatible with the ratio of neutrons in the two targets. Such a linear dependence of $\pi^{-} / \pi^{+}$on $N / Z$ at subthreshold energies is in contrast to the relation found at relativistic energies, although the available data is rather limited at both energies. It is also interesting to note that an anomalously large ratio of $\pi^{-} / \pi^{+} \approx$ 7 has been observed in the reaction of ${ }^{12} \mathrm{C}+{ }^{208} \mathrm{~Pb}$ at $E_{\text {beam }} / A=85 \mathrm{MeV}$ 197. 


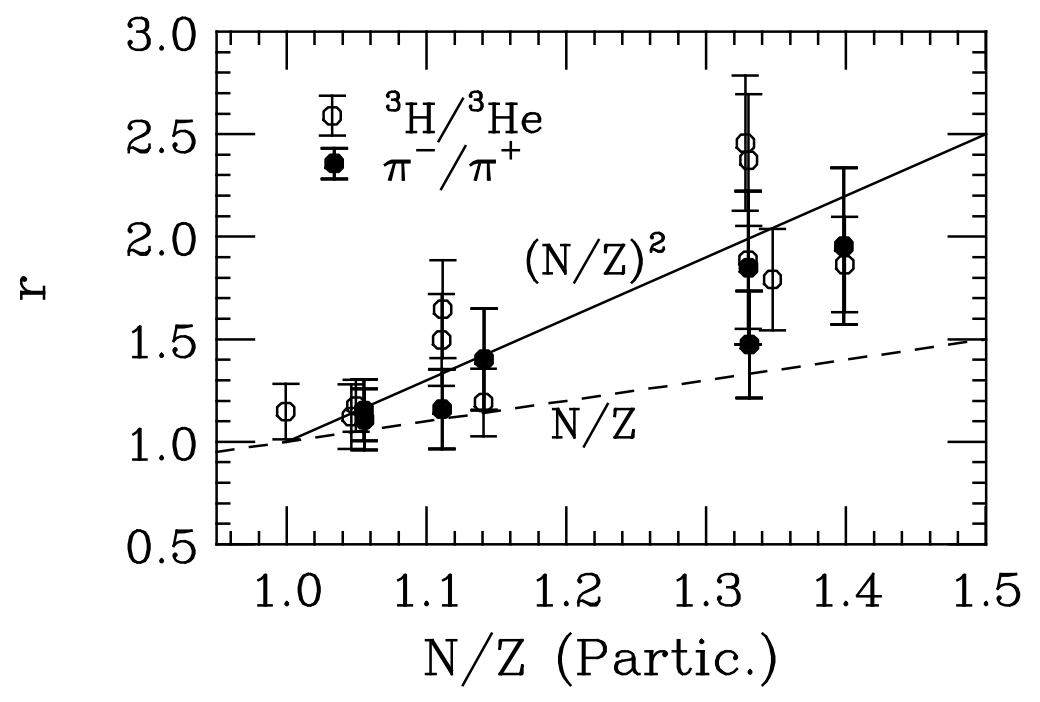

Figure 4.34: The ratio of $\pi^{-} / \pi^{+}$as a function of $N / Z$ ratio of the participants in the reaction of $\mathrm{Ar}+\mathrm{KCl}$. Taken from Ref. [195].

This ratio cannot be explained by the neutron-excess in the ${ }^{208} \mathrm{~Pb}$ target. Thus, the relation between $\pi^{-} / \pi^{+}$and $N / Z$ of the participants has not been well established experimentally at subthreshold energies. The simple scaling law of Eq. (4.11) thus needs to be tested in a wide range of beam energies and $N / Z$ ratios. High intensity beams of neutron-rich or radioactive nuclei are useful for this purpose.

The possibility of using the $\pi^{-} / \pi^{+}$ratio to extract information about the distribution of neutrons and protons in neutron-rich nuclei has been studied using the Glauber model [198, 199, 200. Under the assumption that the nucleon density distribution is a Gaussian function, i.e.,

$$
\rho(r)=\rho(0) \exp \left(-\frac{r^{2}}{a^{2}}\right),
$$

the integration in Eq. (4.8) can be carried out analytically to yield the following result

$$
T(b)=\exp (-\overline{N(b)}),
$$

with the average number of nucleon-nucleon collisions per nucleon in the reaction of $A+B$ at an impact parameter $b$

$$
\overline{N(b)}=\frac{\bar{\sigma} \pi^{2} \rho_{A}(0) \rho_{B}(0) a_{A}^{3} a_{B}^{3}}{a_{A}^{2}+a_{B}^{2}} \cdot \exp \left(-\frac{b^{2}}{a_{A}^{2}+a_{B}^{2}}\right) .
$$

In the above $\bar{\sigma}$ is the isospin-averaged nucleon-nucleon cross section. Similarly, the average number of proton-proton, neutron-neutron and proton-neutron collisions can 
be obtained. If one assumes that pions are produced through $\Delta(1232)$ resonances, the inclusive $\pi^{+}$and $\pi^{-}$cross sections can be written as 199, 201]

$$
\begin{aligned}
\frac{d \sigma_{\mathrm{inc}}^{\pi^{+}}}{d \Omega} & =\left|f_{N \Delta}\right|^{2} Z_{A} Z_{B} \frac{\pi^{2} \rho_{Z_{A}}(0) \rho_{Z_{B}}(0) a_{Z_{A}}^{3} a_{Z_{B}}^{3}}{a_{Z_{A}}^{2}+a_{Z_{B}}^{2}} \\
& \cdot 2 \pi \int_{0}^{\infty} b d b \cdot \exp \left(-\frac{b^{2}}{a_{Z_{A}}^{2}+a_{Z_{B}}^{2}}\right) \cdot P_{s}, \\
\frac{d \sigma_{\mathrm{inc}}^{\pi^{-}}}{d \Omega} & =\left|f_{N \Delta}\right|^{2} N_{A} N_{B} \frac{\pi^{2} \rho_{N_{A}}(0) \rho_{N_{B}}(0) a_{N_{A}}^{3} a_{N_{B}}^{3}}{a_{N_{A}}^{2}+a_{N_{B}}^{2}} \\
& \cdot 2 \pi \int_{0}^{\infty} b d b \cdot \exp \left(-\frac{b^{2}}{a_{N_{A}}^{2}+a_{N_{B}}^{2}}\right) \cdot P_{s},
\end{aligned}
$$

where

$$
P_{s} \equiv \exp \left[-\frac{\bar{\sigma}(A B-1) \rho_{A}(0) \rho_{B}(0) a_{A}^{3} a_{B}^{3}}{a_{A}^{2}+a_{B}^{2}} \cdot \exp \left(-\frac{b^{2}}{a_{A}^{2}+a_{B}^{2}}\right)\right],
$$

is the elastic survival probability, $\rho_{N_{i}}$ and $\rho_{Z_{i}}$ are the neutron and proton densities of the nucleus $i=A$ or $B$, and $f_{N \Delta}$ is the amplitude for the reaction $N+N \rightarrow N+\Delta$. Knowing the density distribution of neutrons and protons in both the target and projectile, one can then calculate the ratio of $\pi^{-} / \pi^{+}$from the above relations. It has been found that this ratio is rather sensitive to the difference between the neutron and proton density distributions. Shown in Table 4.2 are the ratio $E(\bar{\sigma})$ defined as

$$
E \equiv \frac{\sigma_{\text {inc }}^{\pi^{-}}-\sigma_{\text {inc }}^{\pi^{+}}}{\sigma_{\text {inc }}^{\pi^{-}}+\sigma_{\text {inc }}^{\pi^{+}}}
$$

In this calculation [199 density distributions from the binding energy adjusted shell model [192] are used. Results using two isospin-averaged nucleon-nucleon cross sections of $40 \mathrm{mb}$ and $25 \mathrm{mb}$ are compared. It is seen that the ratio $E$ is not so sensitive to the cross section $\bar{\sigma}$, but is sensitive to the difference between the density distributions of neutrons and protons. This prediction has attracted some experimental interest 202] and can be tested in future experiments with high intensity radioactive beams. 
Table 4.2: Normalized difference between $\pi^{-}$and $\pi^{+}$production cross sections for reactions of $\mathrm{Li}$ isotopes on ${ }^{12} \mathrm{C}$

\begin{tabular}{ccccc}
\hline Ratio E $(\bar{\sigma})$ & ${ }^{7} L i+{ }^{12} C$ & ${ }^{8} L i+{ }^{12} C$ & ${ }^{9} L i+{ }^{12} C$ & ${ }^{11} L i+{ }^{12} C$ \\
$\bar{\sigma}=40 \mathrm{mb}$ & 0.1153 & 0.2221 & 0.2955 & 0.3951 \\
$\bar{\sigma}=25 \mathrm{mb}$ & 0.1143 & 0.2210 & 0.2939 & 0.3927 \\
\hline
\end{tabular}




\section{Chapter 5}

\section{Overview and perspectives}

Calculations based on microscopic many-body theories have predicted many interesting new physics phenomena in isospin-asymmetric nuclear matter. These theoretical studies have shown that the equation of state of isospin-asymmetric nuclear matter depends quadratically on the neutron excess $\delta$. The coefficient of the quadratic term in the EOS of asymmetric nuclear matter, i.e., the symmetry energy, and especially its density dependence vary widely among theoretical predictions. In particular, the symmetry energy at high densities suffers the most severe uncertainties among all properties of dense nuclear matter.

Theoretical studies also indicate that the isospin-dependent EOS has significant influence on the properties of neutron stars as well as on those of radioactive nuclei. The softening of the equation of state in isospin-asymmetric nuclear matter is important for the prompt explosion of a type II supernova. The proton concentration in neutron stars at $\beta$ equilibrium is almost completely determined by the symmetry energy. Consequently, the cooling rate and neutrino emissions of neutron stars are affected significantly by the symmetry energy, especially its density dependence. The binding energy, radii and deformation of radioactive nuclei are also shown to depend critically on the isospin-dependence of the nuclear Eos. The nucleon-nucleon cross sections in free space are known to be also strongly isospin-dependent. Nuclear many-body theories have predicted that the isospin-dependence of nucleon-nucleon cross sections might be significantly altered by the nuclear medium.

Because of the additional degree of freedom, isospin, new phenomena are predicted to occur in isospin-asymmetric nuclear matter. In particular, the chemical instability is predicted to occur in a larger configuration space than the mechanical instability. Consequently, the spinodal decomposition in asymmetric nuclear matter might be triggered by fluctuations in the isospin asymmetry rather than baryon density. This may have significant effects on the mechanism for nuclear multifragmentations in nuclear reactions involving isospin asymmetric nuclear matter. The liquid-gas phase transition in asymmetric nuclear matter has been predicted to be second-order rather than first-order as in symmetric nuclear matter. Moreover, the critical temperature of liquid-gas phase transition gradually decreases to zero from symmetric nuclear matter to pure neutron matter.

The predicted new physics phenomena in isospin asymmetric nuclear matter not 
only motivates further theoretical studies but also calls for dedicated experiments to test them. Energetic collisions of stable and radioactive neutron-rich nuclei can create transiently nuclear matter with appreciable isospin asymmetry and matter compression in the overlap region, thus providing a testing ground for studying isospin physics. In fact, many interesting isospin effects have already been observed in heavyion collisions. For example, isospin non-equilibrium has been observed in the angular distribution of isotope ratios. Interesting isospin-dependence of nuclear multifragmentation has been observed from comparing reactions of isospin-symmetric systems and highly isospin-asymmetric ones. Abnormally preferential emission of preequilibrium neutrons have been observed in many experiments. Very recently, the isospindependence of nuclear collective flow and balance energy has been first predicted by using isospin-dependent transport models and later confirmed by experiments. The theoretical understanding of the observed isospin-dependent phenomena has been limited to the qualitative level in most cases. For example, isospin-dependent transport models, though very useful for predicting and explaining many observations qualitatively, are still unable to explain the data quantitatively. Nonetheless, these models are very useful in establishing connections between the experimental observables and the isospin-dependent equation of state and in-medium cross sections. For instance, it has been shown that the neutron/proton ratio of preequilibrium nucleons is very sensitive to the density dependence of the symmetry energy, but not sensitive to the in-medium nucleon-nucleon cross sections. On the other hand, the isospin-dependence of the collective flow and balance energy is mainly caused by the isospin-dependence of the in-medium nucleon-nucleon cross sections.

The main goal of studying isospin physics in heavy-ion collisions is to explore the properties of nuclear matter in the transition region between symmetric nuclear matter and neutron matter and its equation of state. Dedicated radioactive beam facilities will provide excellent opportunities to achieve this goal. We anticipate that there will be substantial experimental activities in studying isospin physics in the coming years. To extract from these experiments more quantitative information about the EOs of asymmetric nuclear matter and isospin-dependence of in-medium nucleon-nucleon cross sections, it is necessary to combine theoretical and computational techniques in the area of nuclear structure, reaction and astrophysics. With a continued dialog between theories and experiments, we believe that this goal will be achieved. 


\section{Chapter 6}

\section{Acknowledgement}

We would like to thank R. Pak, J. Randrup, G.D. Westfall, S.J. Yennello, and Zhongzhou Ren for collaboration in the study of several topics discussed in this review. We are also grateful to J.B. Natowitz, L.G. Sobotka, W.Q. Shen and W.U. Schröder for helpful conversations on the subject. The work of BAL and CMK was supported in part by the NSF Grant No. PHY-9509266 and the Robert A. Welch Foundation under Grant A-1358, and that of WB was supported in part by the NSF Grant No. PHY-9403666 and the NSF Presidential Faculty Fellow grant No. PHY-9253505. 


\section{Bibliography}

[1] North American Steering Committee for the Isospin Laboratory, research opportunities with radioactive nuclear beams. Report LALP91-51.

[2] M.S. Hussein, R.A. Rego and C.A. Bertulani, Phys. Rep. 201, 279 (1991).

[3] R.N. Boyd and I. Tanihata, Phys. Today, 45(6), 45 (1992).

[4] A.C. Müller and B.M. Sherrill, Ann. Rev. Nucl. Part. Sci. 43, 529 (1993).

[5] I. Tanihata, Prog. of Part. and Nucl. Phys., 35 (1995) 505.

[6] H. Geissel, G. Münzenberg, and K. Riisageer, Ann. Rev. Nucl. Part. Sci. 45, 163 (1995).

[7] P.G. Hansen, A.S. Jensen and B. Jonson, Ann. Rev. Nucl. Part. Sci. 45, 591 (1995).

[8] The SPIRAL radioactive ion beam facility, Ganil R 9402, May, 1994.

[9] E. Baron, J. Cooperstein and S. Kahana, Phys. Rev. Lett. 55, 126 (1985); Nucl. Phys. A440, 744 (1985).

[10] S.H. Kahana, Ann. Rev. Nucl. Part. Sci., 39, 231 (1989).

[11] J.M. Lattimer, C.J. Pethick, M. Prakash and P. Haensel, Phys. Rev. Lett. 66, 2701 (1991).

[12] K. Sumiyoshi and H. Toki, Astro. Phys. Journal, 422, 700 (1994).

[13] C-H. Lee, Phys. Rep. 275, 255 (1996).

[14] K.A. Brueckner, S.A. Coon and J. Dabrowski, Phys. Rev. 168, 1184 (1967).

[15] B.D. Serot and J.D. Walecka, Adv. Nucl. Phys. 16, 1 (1986).

[16] H. Müller and B.D. Serot, Phys. Rev. C 52, 2072 (1995).

[17] P.J. Siemens, Nucl. Phys. A141, 225 (1970).

[18] O. Sjöberg, Nucl. Phys. A222, 161 (1974). 
[19] J. Cugnon, P. Deneye and A. Lejeune, Z. Phys. A 328, 409 (1987).

[20] I. Bombaci and U. Lombardo, Phys. Rev. C44, 1892 (1991).

[21] H. Müther, M. Prakash and T.L. Ainsworth, Phys. Lett. B199, 469 (1987).

[22] B. ter Haar and R. Malfliet, Phys. Rev. C50, 31 (1994).

[23] K. Sumiyoshi, H. Toki and R. Brockmann, Phys. Lett. B276, 393 (1992).

[24] H. Huber, F. Weber and M.K. Weigel, Phys. Lett. B317, 485 (1993); Phys. Rev. C50, R1287 (1994).

[25] B. Friedman and V.R. Pandharipande, Nucl. Phys. A361, 502 (1981).

[26] I.E. Lagaris and V.R. Pandharipande, Nucl. Phys. A369, 470 (1981)

[27] R.B. Wiringa, V. Fiks and A. Fabrocini, Phys. Rev. C38, 1010 (1988).

[28] S.A. Chin, Ann. Phys. (N.Y.), 108, 301 (1977).

[29] C.J. Horowitz and B.D. Serot, Nucl. Phys. A464, 613 (1987); B.D. Serot and H. Uechi, Ann. Phys. (N.Y.) 179, 272 (1987).

[30] N.K. Glendenning, Phys. Lett. B114, 392 (1982).

[31] D. Hirata et al., Phys. Rev. C44, 1467 (1991).

[32] Y. Sugahara and H. Toki, Nucl. Phys. A579, 557 (1994).

[33] M. Lopez-Quelle et al., Nucl. Phys. A483, 479 (1988).

[34] T.R. Werner et al., Phys. Lett. B333, 303 (1994).

[35] Dao T. Khoa, W. Von Oertzen and A.A. Ogloblin, Nucl. Phys. A602, 98 (1996).

[36] K. Kolehmainen et al., Nucl. Phys. A439, 535 (1985); J. Treiner et al., Ann. Phys. (N.Y.), 170, 406 (1986).

[37] D. Bandyopadhyay, C. Samanta, S.K. Samaddar and J.N. De, Nucl. Phys. A511, 1 (1990).

[38] M. Prakash and T.L. Ainsworth, Phys. Rev. C 36, 346 (1987).

[39] R. Brockmann and R. Machleidt, Phys. Rev. C42, 1965 (1990); H. Müther, R. Machleidt and R. Brockmann, ibid C42, 1981 (1990).

[40] I. Tanihata, Preprint RIKEN-AF-NP-229, July, 1996.

[41] K. Sumiyoshi, D. Hirata, H. Toki and H. Sagawa, Nucl. Phys. A552, 437 (1993).

[42] G. Baym, H.A. Bethe, and C.J. Pethick, Nucl. Phys. A175, 225 (1971). 
[43] V. Thorsson, M. Prakash and J.M. Lattimer, Nucl. Phys. A572, 693 (1994).

[44] M. Prakash, T.L. Ainsworth and J.M. Lattimer, Phys. Rev. Lett. 61, 2518 (1988).

[45] P.E. Haustein, Atomic data and nuclear data tables, 39, 185-395 (1988).

[46] M. Farine, J.M. Pearson and B. Rouben, Nucl. Phys. A304, 317 (1978).

[47] J.M. Pearson et. al., Nucl. Phys. A528, 1 (1991).

[48] P.-G. Reinhard, Z. Phys. A329, 257 (1988).

[49] M. Rufa, P.-G. Reinhard, J. Maruhn, W. Greiner and M.R. Strayer, Phys. Rev. C38, 390 (1988).

[50] M.M. Sharma, M.A. Nagarajan and P. Ring, Phys. Lett. B312, 377 (1993).

[51] J. Schaffner and I.N. Mishustin, Phys. Rev. C53, 1416 (1996).

[52] S. Shlomo and D.H. Youngblood, Phys. Rev. C47, 529 (1993).

[53] N.K. Glendenning, Z. Phys. A326, 57 (1987); ibid, A327, 295 (1987).

[54] M. Kutschera, Phys. Lett. B340,1; Z. Phys. A348, 263 (1994); M. Kutschera and W. Wójcik, Phys. Lett. B223, 11 (1989); Phys. Rev. C47, 1077 (1993).

[55] V.R. Pandharipande and V.K. Garde, Phys. Lett. B39, 608 (1972).

[56] C.J. Pethick and D.G. Ravenhall, Ann. Rev. Nucl. Part. Sci. 45, 429 (1995).

[57] H.A. Bethe, Rev. of Modern Physics, 62, 801 (1990).

[58] M. Prakash, I. Bombaci, M. Prakash, P.J. Ellis, J.M. Lattimer and R. Knorren, Phys. Rep. (1996) in press.

[59] M. Prakash, S. Reddy, J.M. Lattimer and P.J. Ellis, Heavy Ion Physics (1996) in press.

[60] F. Weber and N.K. Glendenning, Lecture notes at the 3rd Mario Schönberg School on Physics, July, 1996, to be published in the Brasilian Journal of Teaching Physics.

[61] K. Sumiyoshi, H. Suzuki and H. Toki, Astronomy and Astrophysics, 303, 475 (1995).

[62] V.S. Uma Maheswari, J.N. De and S.K. Samaddar, Nucl. Phys. A615, 516 (1997).

[63] P. Donati, P.M. Pizzochero, P.F. Bortignon and R.A. Broglia, Phys. Rev. Lett. 72, 2835 (1994). 
[64] D.J. Dean, S.E. Koonin, K.Langanke and P.B. Radha, Phys. Lett. B356, 429 (1995).

[65] L. Engvik et al., Phys. Rev. Lett. 73, 2650 (1994).

[66] M. Prakash, T.L. Ainsworth and J.M. Lattimer, Phys. Rev. Lett. 61, 2518 (1988).

[67] T.R. Werner et al., Phys. Lett. B335, 259 (1994).

[68] H. Scheit et al., Phys., Rev. Lett. 77, 3967 (1996).

[69] T. Glasmacher et al., Phys. Lett. B395, 163 (1997).

[70] M. Farine, T. Sami, B. Remaud and F. Sébille, Z. Phys. A339, 363 (1991).

[71] L.G. Sobotka, Phys. Rev. C50, R1272 (1994).

[72] Y. Aboussir et al., Nucl. Phys. A549, 155 (1992).

[73] P. Danielewicz, Phys. Rev. C46, 2002 (1992).

[74] B.A. Li and J. Randrup, HMI preprint (1993).

[75] B. Jouault et al., Preprint SUBATECH-95-11.

[76] W.D. Myers and W.J. Swiatecki, Ann. of Phys. (N.Y.), 84, 186 (1974).

[77] J.M. Lattimer and D.G. Ravenhall, Astrophysical Journal, 223, 314 (1978).

[78] M. Barranco and J.R. Buchler, Phys. Rev. C22, 1729 (1980).

[79] L.G. Moretto et al., Phys. Rev. Lett. 69, 1884 (1992).

[80] W. Bauer, G.F. Bertsch, and H. Schulz, Phys. Rev. Lett. 69, 1888 (1992); L. Phair, W. Bauer, and C.K. Gelbke, Phys. Lett. B314, 271 (1993).

[81] D. Gross, Bao-An Li and A.R. DeAngelis, Ann. Phys. 1, 467 (1992); Bao-An Li and D.H.E. Gross, Nucl. Phys. A554, 257 (1993).

[82] B. Borderie et al., Phys. Lett. B302, 15 (1993).

[83] L.G. Moretto and G.J. Wozniak, Ann. Rev. Nucl. Part. Sci. 43, 123 (1993).

[84] M. Brack, C. Guet and H.-B Hákansson, Phys. Report, 123, 275 (1985).

[85] H.R. Jaqman, A.Z. Mekjian and L. Zamick, Phys. ReV. C27, 2782 (1983); ibid C29, 2067 (1984).

[86] H.R. Jaqaman, Phys. ReV. C39, 169 (1988); ibid C40, 1677 (1989).

[87] B.A Li and C. M. Ko, Nucl. Phys. A618, 498 (1997). 
[88] C. Gale, G.F. Bertsch and S. Das Gupta, Phys. Rev. C35, 1666 (1987).

[89] J. Zhang, S. Das Gupta and C. Gale, Phys. Rev. C50, 1617 (1994).

[90] C. Gale, G.M. Welke, M Prakash, S.J. Lee, and S. Das Gupta, Phys. Rev. C41, 1545 (1990).

[91] B.A. Li, C.M. Ko and Z.Z. Ren, Phys. Rev. Lett. 78, 1644 (1997).

[92] L.P. Csernai, G. Fai, C. Gale and E. Osnes, Phys. Rev. C 46, 736 (1992).

[93] V.K. Mishra, G. Fai, L.P. Csernai and E. Osnes, Phys. Rev. C47, 1519 (1993).

[94] J.F. Dempsey et al., Phys. Rev. C 54, 1710 (1996).

[95] G.J. Kunde et al., Phys. Rev. Lett. 77, 2897 (1996).

[96] J. Toke et al., Phys. Rev. Lett. 75, 2920 (1996).

[97] L.G. Sobotka, J.F. Dempsey, R.J. Charity, and P. Danielewicz, Phys. Rev. C 55, 2109 (1997).

[98] G. Kortmeyer, W. Bauer, and G.J. Kunde, Phys. Rev. C 55, 2730 (1997).

[99] G.F. Bertsch and P.J. Siemens, Phys. Lett. B126, 9 (1983).

[100] S. Ayik and C. Gregoire, Phys. Lett. B212, 269, (1988); Nucl. Phys. A513, 187 (1990).

[101] J. Randrup and B. Remaud, Nucl. Phys. A514, 339 (1990).

[102] Ph. Chomaz, G.F. Burgio and J. Randrup; Phys. Lett. B254, 340 (1991); G.F. Burgio, Ph. Chomaz and J. Randrup; Nucl. Phys. A529, 157 91991); Phys. Rev. Lett., 69, 1888 (1992).

[103] M. Colonna, Ph. Chomaz and J. Randrup, Nucl. Phys. A567, 637 (1994); M. Colonna and Ph. Chomaz, Phys. Rev. C49, 1908 (1994).

[104] S. Ayik and J. Randrup, Phys. Rev. C50, 2947 (1994).

[105] I. Hamamoto and H. Sagawa, Phys. Rev. C53, R1492 (1996).

[106] M. Colonna, M. Di Toro and A.B. Larionov, Catania preprint, 1997.

[107] D.Q. Lamb, J.M. Lattimer, C.J. Pethick and D.G. Ravenhall, Nucl. Phys. A360, 459 (1981).

[108] J.M. Lattimer, C.J. Pethick, D.G. Ravenhall and D.Q. Lamb, Nucl. Phys. A432, 646 (1985).

[109] N.K. Glendenning, Phys. Rev. D46, 1274 (1992). 
[110] T.T.S. Kuo, S. Ray, J. Shamanna and R.K. Su, Int. Jour. of Modern Phys. E: Nucl. Phys. 5, 303 (1996); S. Ray, J. Shamanna and T.T.S. Kuo, Phys. Lett. B (1996) in press; T.T.S. Kuo, S. Ray, J. Shamanna, R.K. Su, SUNY-preprint, 1996

[111] S.K. Charagi and S.K. Gupta, Phys. Rev. C41, 1610 (1990).

[112] G. Alkahzov et al., Nucl. Phys. A280, 365 (1977).

[113] G.F. Bertsch, G.E. Brown, V. Koch and Bao-An Li, Nucl. Phys. A490, 745 (1988).

[114] A. Bohnet et al., Nucl. Phys. A494, 349 (1989).

[115] A. Faessler, Nucl. Phys. A495, 103c (1989).

[116] H.S. Köhler, Nucl. Phys. A529, 209 (1991).

[117] G.Q. Li and R. Machleidt, Phy. Rev. C48, 1702 (1993); ibid, C49, 566 (1994).

[118] T. Alm, G. Röpke and M. Schmidt, Phys. Rev. C50 (1994) 31.

[119] T. Alm, G. Röpke, W. Bauer, F. Daffin and M. Schmidt, Nucl. Phys. A587, 815 (1995).

[120] G.D. Westfall et al., Phys. Rev. Lett. 71, 1986 (1993).

[121] D. Klakow, G. Welke and W. Bauer, Phys. Rev. C48, 1982 (1993).

[122] M.J. Huang et al., Phys. Rev. Lett. 77, 3739 (1996).

[123] S.J. Yennello et al., in Proc. of International Workshop on Physics of Unstable Nuclear beams, Serra Negra, Brazil, Aug. 28-31, 1996. (World Scientific, Singapore).

[124] B. Remaud, C. Grëgoire, F. Sëbille and P. Schuck, Nucl. Phys. A488 (1988) 423c.

[125] C. Hartnack, H. Stöcker and W. Greiner, in Proceedings of the International Workshop on Gross Properties of Nuclei and Nuclear Excitation XVI, Hirschegg, Austria, 1988, ed. H. Feldmeier, p.138.

[126] M.B. Tsang, G.F. Bertsch, W.G. Lynch and M. Tohyama, Phys. Rev. C40 (1989) 1685.

[127] B.A. Li and W. Bauer, Phys. Lett. B254, 335 (1991); Phys. Rev. C44, 450 (1991).

[128] B.A. Li, W. Bauer and G.F. Bertsch, Phys. Rev. C44, 2095 (1991).

[129] B.A. Li and S.J. Yennello, Phys. Rev. C52, R1746 (1995). 
[130] B.A. Li, Z.Z. Ren, C.M. Ko and S.J. Yennello, Phys. Rev. Lett. 76, 4492 (1996).

[131] H. Stöcker and W. Greiner, Phys. Rep. 137, 277 (1986).

[132] G.F. Bertsch and S. Das Gupta, Phys. Rep. 160, 189 (1988).

[133] W. Cassing, V. Metag, U. Mosel and K. Niita, Phys. Rep. 188, 363 (1990).

[134] W. Bauer, C.K. Gelbke, and S. Pratt, Ann. Rev. Nucl. Part. Sci. 42 (1992) 77.

[135] J. Aichelin, Phys. Rep. 202, 233 (1991).

[136] H.M. Xu, Phys. Rev. Lett. 67, 2769 (1992); Phys. Rev. C46, R392 (1992).

[137] B.A. Li, Phys. Rev. C48, 2415 (1993).

[138] J.A. Christley et al., Nucl. Phys. A587, 390 (1995).

[139] P.E. Hodgson, Phys. Lett. 6, 75 (1963).

[140] H. Lenske, H.H. Wolter and H.G. Bohlen, Phys. Rev. Lett. 62, 1457 (1989).

[141] W.U. Schröder, private communication.

[142] Z.Z. Ren, W. Mittig, B.Q. Chen, and Z.Y. Ma, Phys. Rev. C52, R20 (1995).

[143] D. Hilscher et al., Phys. Rev. C36, 208 (1987).

[144] D. Hilscher, in Proc. of a Specialists's Meeting on Preequilibrium Nuclear Reactions, Semmering, Austria, 10-12th, Feb. 1988, Ed. B. Strohmaier (OECD, Paris, 1988), p. 245.

[145] D. Polster et al., in Book of abstracts, International Nuclear Physics Conference, Wiesbaden, Germany, July 26-Aug. 1, 1992, p3.3.8.

[146] D. Polster et al., Phys. Rev. C51, 1167 (1995).

[147] D.K. Agnihotri et al., in Proceedings of the $13^{\text {th }}$ Winter Workshop of Nuclear Dynamics, Marathon, Florida, Feb. 1997, Eds. W. Bauer and A. Mignerey, Plenum Press, to be published.

[148] M. Gonin et al., Phys. Rev. C42, 2125 (1990).

[149] K.A. Hanold et al., Phys. Rev. C52, 1462 (1995).

[150] S.A. Bass, J. Konopka, M. Bleicher, H. Stöcker and W. Greiner, GSI annual report, p. 66 (1994).

[151] S.J. Yennello, B. Young, J. Yee, J.A. Winger, J. S. Winfield, G.D. Westfall, A. Vander Molen, B.M. Sherrill, J. Shea, E. Norbeck, D.J. Morrissey, T. Li, E. Gualtieri, D. Craig, W. Benenson and D. Bazin, Phys. Lett. B321 (1994) 15. 
[152] S. J. Yennello, J.A. Winger, H. Johnston, T. White, E. Gualtieri, D. Craig, S. Hannuschke, J. Yee, R. Pak, A. Vander Molen, W. Benenson, G.D. Westfall, T. Li, W.J. Liope, D. J. Morrissey, J. S. Winfield and M. Steiner, TAMU Progress in Research, P. I-7, 1994.

[153] H. Johnston, J. Winger, T. White, B. Hurst, D. O'Kelly and S. J. Yennello, Phys. Lett. B371, 186 (1996).

[154] H. Johnston, T. White, Bao-An Li, E. Ramakrishnan, J. Winger, D.J. Rowland, B. Hurst, F. Gimeno-Nogues, D. O’Kelly, Y-W. Lui and S. J. Yennello, Phys. Rev. C, (1997) in press.

[155] Y. Yariv and Z. Frankel, Phys. Rev. C26 (1982) 2138.

[156] B. Gatty et al., Z. Phys. A273 (1975) 65.

[157] B. Borderie et al., in: Proc. of ACS Nuclear Chemistry Award Symposium, Anaheim, USA, April 2-4, 1995.

[158] A. Ono and H. Horiuchi, Phys. Rev. C53, 2958 (1996).

[159] W. Bauer, Phys. Rev. Lett. 61, 2534 (1988).

[160] S. Das Gupta and G.D. Westfall, Physics Today, 46(5), 34 (1993).

[161] D. Krofcheck et al., Phys. Rev. Lett. 63, 2028 (1989).

[162] C.A. Ogilvie et al., Phys. Rev. C40, 2592; ibid, C42, R10 (1990);

Phys. Lett. B231, 35 (1989).

[163] J. Péter et al., Phys. Lett. B237, 187 (1990).

[164] J.P. Sullivan et al., Phys. Lett. B249, 8 (1990).

[165] J. Péter et al., Nucl. Phys. A519, 611 (1990).

[166] W.M. Zhang et al., Phys. Rev. C42, R491 (1990).

[167] D. Krofcheck et al., Phys. Rev. C43, 350 (1991); ibid 46, 1416 (1992).

[168] W.K. Wilson et al., Phys. Rev. C45, 768 (1992).

[169] G.D. Westfall et al., Phys. Rev. Lett. 71, 1986 (1993).

[170] P. Danielewicz and G. Odyniec, Phys. Lett. B157, 146 (1985).

[171] J. Molitoris and H. Stöcker, Phys. Lett. B162, 47 (1985).

[172] J. Molitoris, D. Hahn and H. Stöcker, Nucl. Phys. A447, 13c (1986).

[173] G.F. Bertsch, W.G. Lynch and M.B. Tsang, Phys. Lett. B189, 738 (1987). 
[174] P. Danielewicz et al., Phys. Rev. C38, 120 (1989).

[175] V. de la Mota, F. Sebille, M. Farine, B. Remaud and P. Schuck, Phys. Rev. C46, 677 (1992).

[176] Q. Pan and P. Danielewicz, Phys. Rev. Lett. 70, 2062 (1993).

[177] P. Danielewicz, private communication.

[178] R. Pak et al., Phys. Rev. Lett. 781022 (1997).

[179] R. Pak et al., Phys. Rev. Lett. 781026 (1997).

[180] R. Pak et al., Phys. Rev. C53, 1469 (1996).

[181] S. Kox et al.. Phys. Rev. C35, 1678 (1987).

[182] K. Yabana, Y. Ogawa and Y. Suzuki, Nucl. Phys. A539, 295 (1992).

[183] Y. Ogawa, K. Yabana and Y. Suzuki, Nucl. Phys. A543, 722 (1992).

[184] W.Q. Shen et al., Nucl. Phys. A491, 130 (1989).

[185] P.J. Karol, Phys. Rev. C11, 1203 (1975).

[186] W. Mittig et al., Phys. Rev. Lett. 59, 1889 (1987).

[187] T. Suzuki et al., Phys. Rev. Lett. 75, 3241 (1995).

[188] A.J. Cole, Z. Phys. A322, 315 (1985); Phys. Rev. C35, 117 (1987); ibid, C40, 1265 (1989).

[189] B.G. Harvey, Nucl. Phys. A444, 498 (1985).

[190] A .D. Jackson and M. Boggild, Nucl. Phys. A470, 669 (1987).

[191] Y.Q. Ma et al., Phys. Lett. B302, 386 (1993); Phys. Rev. C48, 850 (1993).

[192] G.F. Bertsch, B.A. Brown and H. Sagawa, Phys. Rev. C39, 1154 (1989).

[193] R. Stock, Phys. Report, 135, 259 (1986).

[194] B.A. Li, Phys. Lett. B346, 5 (1995).

[195] S. Nagamiya, Phys. Rev. C24, 971 (1981).

[196] B. Noren et al., Nucl. Phys. A489, 763 (1988).

[197] V. Bernard et al., Nucl. Phys. A423, 511 (1984).

[198] R.J. Lombard and J.P. Maillet, Europhys. Lett. 6, 323 (1988).

[199] B.A. Li, M.S. Hussein and W. Bauer, Nucl. Phys. A533, 749 (1991). 
[200] W. Bauer et al., in Proceedings of the Workshop on the Science of Intensive Radioactive Ion Beams, Los Alamos report LA-11964-C, p. 57 (1990).

[201] A. Tellez, R.J. Lombard and J.P. Maillet, J. of Phys. G13, 311 (1987).

[202] B.M. Sherrill, Advances in Nuclear Dynamics, eds. J. Harris, A. Mignerey and W. Bauer, (World scientific, Singapore), 1994. P.70. 